\title{
Higgs low-energy theorem (and its corrections) in composite models
}

\author{
M. Gillioz, ${ }^{a}$ R. Gröber, ${ }^{b}$ C. Grojean, ${ }^{c}$ M. Mühlleitner ${ }^{b}$ and E. Salvioni ${ }^{c, d}$ \\ ${ }^{a}$ Institut für Theoretische Physik, Universität Zürich, \\ CH-8051 Zürich, Switzerland \\ ${ }^{b}$ Institut für Theoretische Physik, Karlsruhe Institute of Technology, \\ D-76128 Karlsruhe, Germany \\ ${ }^{c}$ Theory Division, Physics Department, CERN, \\ CH-1211 Geneva 23, Switzerland \\ ${ }^{d}$ Dipartimento di Fisica e Astronomia, Università di Padova and INFN, \\ Via Marzolo 8, \\ I-35131 Padova, Italy \\ E-mail: gillioz@cp3.dias.sdu.dk, ramona@particle.uni-karlsruhe.de, \\ christophe.grojean@cern.ch, maggie@particle.uni-karlsruhe.de, \\ ennio.salvioni@cern.ch
}

ABSTRACT: The Higgs low-energy theorem gives a simple and elegant way to estimate the couplings of the Higgs boson to massless gluons and photons induced by loops of heavy particles. We extend this theorem to take into account possible nonlinear Higgs interactions as well as new states resulting from a strong dynamics at the origin of the breaking of the electroweak symmetry. We show that, while it approximates with an accuracy of order a few percents single Higgs production, it receives corrections of order $50 \%$ for double Higgs production. A full one-loop computation of the $g g \rightarrow h h$ cross section is explicitly performed in MCHM5, the minimal composite Higgs model based on the $\mathrm{SO}(5) / \mathrm{SO}(4)$ coset with the Standard Model fermions embedded into the fundamental representation of $\mathrm{SO}(5)$. In particular we take into account the contributions of all fermionic resonances, which give sizeable (negative) corrections to the result obtained considering only the Higgs nonlinearities. Constraints from electroweak precision and flavor data on the top partners are analyzed in detail, as well as direct searches at the LHC for these new fermions called to play a crucial role in the electroweak symmetry breaking dynamics.

Keywords: Higgs Physics, Beyond Standard Model, Technicolor and Composite Models ARXIV EPRINT: 1206.7120 


\section{Contents}

1 Introduction 1

2 Low-energy effective Lagrangian for a composite Higgs boson 3

3 Applying the Higgs low-energy theorem 5

$\begin{array}{ll}3.1 \text { Higgs interactions with gluons } & 6\end{array}$

3.2 Higgs interaction with photons 8

3.3 Single Higgs production via gluon fusion $\quad 9$

3.4 Double Higgs production via gluon fusion $\quad 11$

4 Composite Higgs model with extra fermionic resonances $\quad \mathbf{1 5}$

$\begin{array}{lll}\text { 4.1 Constraints from electroweak precision data and flavor physics } & 18\end{array}$

$\begin{array}{lll}4.2 & \text { Constraints from searches for heavy fermions } & 21\end{array}$

5 Single Higgs production in MCHM5 24

$\begin{array}{lll}5.1 & \text { Effect of non-minimal operators } & 25\end{array}$

6 Double Higgs production in MCHM5 27

$\begin{array}{lll}6.1 & \text { LET cross section } & 27\end{array}$

6.2 Enhancement of the cross section 27

$\begin{array}{lll}6.3 & \text { Full 1-loop cross section } & 28\end{array}$

$\begin{array}{lll}6.4 & \text { Numerical analysis } & 29\end{array}$

$\begin{array}{lll}7 & \text { Conclusions } & 32\end{array}$

A Derivation of the $h g g, h h g g$ and $h \gamma \gamma$ couplings in the SILH formalism $\quad 33$

A.1 The $h g g$ and hhgg couplings 33

$\begin{array}{lll}\text { A.2 The } h \gamma \gamma \text { coupling } & 34\end{array}$

B The SILH coefficients for the Littlest Higgs and MCHM4 35

B.1 Littlest Higgs $\quad 35$

$\begin{array}{lll}\text { B.2 } & \text { MCHM4 } & 38\end{array}$

C The $\chi^{2}$ test for electroweak precision observables 38

D Partial decay widths of heavy fermions in MCHM5 40

D.1 Charge $2 / 3$ states 41

D.2 $B$ and $X^{5 / 3} \quad 42$ 
E Analytical results for the $g g \rightarrow h h$ cross section in MCHM5

E.1 Notation 42

E.2 Tensor basis and projectors 43

E.3 Triangle form factor 43

E.4 Box form factors 43

E.5 Expansion of the form factors 45

\section{Introduction}

There is growing evidence that a Higgs boson is the agent of electroweak symmetry breaking (EWSB) [1, 2]. And one of the most pressing questions is to uncover the true nature of this Higgs boson: is it the elementary scalar field of the Standard Model? Is it part of a supermultiplet? Is it a composite scalar emerging as a bound state from a strongly coupled sector? Theoretical arguments based on naturalness considerations tend to favor one of the two latter scenarios. But the LHC experiments have now opened a new datadriven era and the first experimental indications might come from possible deviations in the measurements of the Higgs couplings [3-8] compared to the ones predicted by the Standard Model (SM) that are unambiguously fixed by the value of the Higgs mass itself. However, both supersymmetric and composite Higgs bosons in the decoupling limits can be arbitrarily close to the SM Higgs at the energy scale currently probed by the LHC and deciphering the different scenarios from one another might require more luminosity than the one currently accumulated. A more unambiguous answer would come from a direct observation of additional particles: supersymmetric partners of the SM particles or additional resonances of the strong sector. In both scenarios, new particles in the top sector have a special status: in supersymmetric models, the stops cannot be too heavy without destabilizing the weak scale [9]; the top partners in composite models are responsible for generating the potential of the would-be Goldstone Higgs boson and, as recently noticed in refs. [10-13], they have to be lighter than about $700 \mathrm{GeV}$ to naturally accommodate a Higgs boson as light as $125 \mathrm{GeV}$. Even if the actual numbers are model-dependent, this conclusion is rather generic and certainly calls for improving the ongoing dedicated direct searches for the top partners [14-22].

In principle, indirect information on these top partners could also be obtained in Higgs physics. It is indeed quite ironic that, while the Higgs boson is supposed to be at the origin of the masses of all elementary particles, the currently most sensitive channels are the ones that involve massless particles, i.e. particles with no direct coupling to the Higgs boson: the gluons for its production and the photons for its decay. Clearly these processes appear only at the loop level in the SM and are therefore potentially sensitive to new states circulating in the loops. The structure of the Higgs couplings to photons and gluons are beautifully captured by the background field method known as Low-Energy Theorem (LET) [23-25] and the effects of the top partners, or any new particles, are encoded by their sole contributions to the QED and QCD beta functions. In composite Higgs models, the 
corrections due to the Higgs compositeness to the SM gluon fusion production cross section, $\sigma(g g \rightarrow h)$, and to the SM decay width into two photons, $\Gamma(h \rightarrow \gamma \gamma)$, were estimated in ref. [26] to be generically of the order of $\xi=(v / f)^{2}$, where $v \sim 246 \mathrm{GeV}$ is the weak scale and $f$ is the characteristic scale of the strong sector (the equivalent of $f_{\pi}$ in QCD). From dimensional arguments, the additional corrections due to the top partners should be of the order of $\left(m_{t} / m_{T}\right)^{2}, m_{t, T}$ being respectively the mass of the top and the typical mass scale of his partners, i.e. a correction potentially as big as the one originating from the strong dynamics itself when the top partners are around $700 \mathrm{GeV}$. However, it was quickly realized [27-29] that these leading-order corrections from the top partners actually cancel and that they only give a contribution that we shall estimate (see section 3.3) to scale like $\xi\left(m_{h} / m_{t}\right)^{2} / 10$, i.e. one order of magnitude smaller than the strong dynamics contribution, sweeping any hope to learn anything about the top partners from the measurement of the Higgs production by gluon fusion and leaving only the Higgs production in association with a top-antitop pair $[30,31]$ as a place to indirectly look for new physics in the first LHC run. A proper effective description of the top partners/fermionic resonances would be needed to study this promising channel.

The second LHC run will certainly increase the sensitivity of the direct searches for top partners. But it will also open new possibilities to indirectly probe the top partner sector in Higgs physics by exploring multi-Higgs production. A generalization of the LET exists for double Higgs production in the SM, and it is known to give a reasonably good estimate of the total rate within $20 \%$ accuracy for a light Higgs [32, 33] but it badly fails to reproduce the differential distributions [34]. The extension of the LET to strong EWSB models is not totally trivial since, in the SM, there is a strong destructive interference between two contributions and therefore strong dynamics that gives rise to a third new contribution can have order-one effects on the double Higgs production by gluon fusion, $g g \rightarrow h h$, as already noticed in refs. [35-37]. In an explicit composite Higgs model, we are going to compare the LET results to an explicit one-loop computation taking into account all the contributions from the fermionic resonances in the top sector. We will find that the LET is less accurate than in the SM, and underestimates the full result by up to $50 \%$. On the other hand the pure strong dynamics effects arising due to the sole Higgs nonlinearities are overestimating the production rate which receives $\mathcal{O}(30 \%)$ corrections from the top partners.

The outline of our paper is as follows. In section 2, we first present two equivalent effective Lagrangians, the linearly realized strongly-interacting light Higgs Lagrangian [26] as well as a chiral Lagrangian where the $\mathrm{SU}(2)_{L} \times \mathrm{U}(1)_{Y} \mathrm{SM}$ gauge symmetry is nonlinearly realized [38], to describe Higgs physics at the LHC and we relate these effective Lagrangians to explicit composite Higgs models. In section 3, we recall the LET in the SM and extend it to composite Higgs models, reproducing the often heard results that the gluon fusion production cross sections can be significantly reduced by up to $50 \% \div 70 \%$ compared to the SM production. We also re-discuss within the LET approximation the cancellation of the top partner contributions to single Higgs production by gluon fusion and we estimate the size of the corrections to the LET results. We finally derive the LET for double Higgs production by gluon fusion and show that a factor $2 \div 3$ enhancement over the SM can be obtained in specific examples. This LET enhancement factor is nonetheless smaller than 
the one obtained considering only the pure Higgs nonlinearities effects. Section 4 is devoted to the top partners in an explicit minimal composite Higgs model, MCHM5, based on the coset space $\mathrm{SO}(5) / \mathrm{SO}(4)$ with $\mathrm{SM}$ fermions embedded into fundamental representations of $\mathrm{SO}(5)$. We first discuss in detail the constraints on the masses and couplings of these new fermions coming from electroweak (EW) precision measurements and from flavor physics. We then present exhaustive constraints from direct searches for these top partners using the current Tevatron and LHC data. In section 5, a detailed computation of the contribution of the top partners to $\sigma(g g \rightarrow h)$ is presented, confirming that the LET gives a good approximation and confirming the scaling behavior of the corrections obtained in section 3 . Finally, section 6 is devoted to double Higgs production. We demonstrate explicitly that the full loop computation with the top partners can easily give negative corrections of order $30 \%$ to the pure strong dynamics computation where only the Higgs nonlinearities were taken into account [36]. Contrary to the single Higgs production case, the LET does not capture well these contributions of the top partners, and this discrepancy is further amplified when looking at particular regions of the phase space selected by kinematical cuts. Various technical details are collected in a series of appendices.

\section{Low-energy effective Lagrangian for a composite Higgs boson}

An interesting solution to the hierarchy problem is given by the Higgs boson being a composite bound state emerging from a new strongly-interacting sector, broadly characterized by a mass scale $m_{\rho}$ and a coupling $g_{\rho}$. If in addition the Higgs emerges as a pseudoGoldstone boson of a spontaneous symmetry breaking $\mathcal{G} / \mathcal{H}$ at the scale $f=m_{\rho} / g_{\rho}$, then it can be naturally lighter than the other resonances of the strong sector and $v^{2} / f^{2} \ll 1$ can be accommodated. A low-energy, model-independent description of this idea is given by the strongly-interacting light Higgs (SILH) Lagrangian [26], which applies to the general scenario where the Higgs is a light pseudo-Goldstone boson, including Little Higgs and Holographic composite Higgs models. At scales much smaller than $m_{\rho}$, deviations from the SM are parameterized in terms of a set of dimension-six operators. The subset that will be relevant in our discussion is

$$
\begin{aligned}
\mathcal{L}_{\mathrm{SILH}}= & \frac{c_{H}}{2 f^{2}} \partial^{\mu}\left(H^{\dagger} H\right) \partial_{\mu}\left(H^{\dagger} H\right)+\frac{c_{r}}{2 f^{2}} H^{\dagger} H\left(D_{\mu} H\right)^{\dagger}\left(D^{\mu} H\right)-\frac{c_{6} \lambda}{f^{2}}\left(H^{\dagger} H\right)^{3} \\
& +\left(\frac{c_{y} y_{f}}{f^{2}} H^{\dagger} H \bar{f}_{L} H f_{R}+\text { h.c. }\right)+\frac{c_{g} g_{s}^{2}}{16 \pi^{2} f^{2}} \frac{y_{t}^{2}}{g_{\rho}^{2}} H^{\dagger} H G_{\mu \nu}^{a} G^{a \mu \nu}+\frac{c_{\gamma} g^{\prime 2}}{16 \pi^{2} f^{2}} \frac{g^{2}}{g_{\rho}^{2}} H^{\dagger} H B_{\mu \nu} B^{\mu \nu}
\end{aligned}
$$

where $g_{s}, g, g^{\prime}$ are the $\mathrm{SM} \mathrm{SU}(3)_{c} \times \mathrm{SU}(2)_{L} \times \mathrm{U}(1)_{Y}$ gauge couplings, whereas $\lambda$ and $y_{f}$ are the Higgs quartic and Yukawa coupling appearing in the SM Lagrangian, respectively. The first four operators are genuinely sensitive to the strong interaction, whereas the last two parameterize the effective couplings of the Higgs to gluons and to photons, respectively, mediated by loops of heavy particles. As the operators proportional to $c_{g}$ and $c_{\gamma}$ do not respect the symmetry under which the Goldstone Higgs shifts they have to be suppressed by powers of the couplings which break this symmetry, thus explaining the extra factor $g_{\mathrm{SM}}^{2} / g_{\rho}^{2}\left(g_{\mathrm{SM}}=y_{t}, g\right)$ appearing in front of them. These operators are important in the 
presence of relatively light resonances, i.e. when $g_{\rho} \sim g_{\mathrm{SM}}$. Recent analyses [10-13] show that in a large class of models, a Higgs as light as $125 \mathrm{GeV}$ implies the presence of one or more anomalously light (sub-TeV) fermionic resonances, which thus can contribute sizeably to $c_{g}$ and $c_{\gamma}$. Also notice that by choosing flavor-diagonal couplings $y_{f}$ Minimal Flavor Violation (MFV) is automatically implemented in the Lagrangian so that it complies with flavor bounds.

In eq. (2.1) we have kept explicitly the operator proportional to $c_{r}$, which can be eliminated at $\mathcal{O}\left(1 / f^{2}\right)$ by a field redefinition

$$
H \rightarrow H+a\left(H^{\dagger} H\right) H / f^{2},
$$

under which

$$
c_{H} \rightarrow c_{H}+2 a, \quad c_{r} \rightarrow c_{r}+4 a, \quad c_{6} \rightarrow c_{6}+4 a, \quad c_{y} \rightarrow c_{y}-a,
$$

while $c_{g}$ and $c_{\gamma}$ do not change under this transformation. The choice $c_{r}=0$ corresponds to the 'SILH basis', which can be reached starting from a generic basis where $c_{r} \neq 0$ by applying the transformation in eq. (2.2) with $a=-c_{r} / 4$. We choose to keep explicitly the operator proportional to $c_{r}$ as the 'natural' basis for nonlinear $\sigma$-models actually corresponds to a non-vanishing $c_{r}$ [28]. Furthermore, since physical amplitudes have to be invariant under field redefinitions, eqs. (2.2) and (2.3) will be used as a consistency check of our results. In eq. (2.1) we have also omitted the custodial breaking operator

$$
\frac{c_{T}}{2 f^{2}}\left(H \overleftrightarrow{D^{\mu}} H\right)^{2}
$$

where $H^{\dagger} \overleftrightarrow{D^{\mu}} H \equiv H^{\dagger}\left(D^{\mu} H\right)-\left(D^{\mu} H\right)^{\dagger} H$, which gives a contribution to the $T$ parameter $\hat{T}=c_{T} v^{2} / f^{2}$ and thus is strongly constrained by electroweak data. This operator does not contribute to the processes that we will be interested in. If the strong sector is invariant under custodial symmetry, as it happens for example in models based on the coset $\mathrm{SO}(5) / \mathrm{SO}(4)$, then $c_{T}$ vanishes at tree-level. For a discussion of Higgs physics where the assumption of custodial invariance is relaxed, see ref. [39].

The SILH Lagrangian represents an expansion in $\xi \equiv(v / f)^{2}$ and can be used in the vicinity of the SM, which corresponds to $\xi=0$. On the other hand, the technicolor limit $(\xi \rightarrow 1)$ requires the resummation of the full series in $\xi$. Such a resummation is possible in the Holographic Higgs models of refs. [40-42]. These models are based on a five-dimensional gauge theory in Anti-de-Sitter (AdS) space-time. In the minimal realization, the bulk symmetry $\mathrm{SO}(5) \times \mathrm{U}(1)$ is broken to the $\mathrm{SM}$ group $\mathrm{SU}(2)_{L} \times \mathrm{U}(1)_{Y}$ on the ultraviolet $(\mathrm{UV})$ brane and to $\mathrm{SO}(4) \times \mathrm{U}(1)$ on the infrared (IR) brane. The coset $\mathrm{SO}(5) / \mathrm{SO}(4)$ provides four Goldstone bosons, one of which is the physical Higgs boson and the three remaining ones are eaten by the massive SM vector bosons. The Higgs couplings to gauge bosons and its self-interactions are modified compared to the SM, and the modification factors can be expressed in terms of the parameter $\xi$. The Higgs Yukawa couplings and the form of the Higgs potential of the low-energy effective theory depend on the way the SM fermions are embedded into representations of the bulk symmetry. In the second part of this work 
we refer to the model MCHM5 [41] where the fermions transform in the fundamental representation of $\mathrm{SO}(5)$. An alternative realization of the $\mathrm{SO}(5) / \mathrm{SO}(4)$ composite Higgs, denoted by MCHM4, contains fermions embedded into the spinorial representation [40] (for more details see appendix B.2). In this case, however, large corrections to the $Z b_{L} b_{L}$ coupling are present and rule out an important part of the parameter space [43]. In contrast, if fermions are embedded into the fundamental or adjoint representation of $\mathrm{SO}(5)$, the custodial symmetry of the strong sector includes a left-right parity, which protects the $Z b_{L} b_{L}$ coupling from receiving tree-level corrections [44].

Another useful description of the low-energy theory is given by an effective chiral Lagrangian where the $\mathrm{SU}(2) \times \mathrm{U}(1)_{Y}$ symmetry is nonlinearly realized. The Goldstone bosons $\pi^{a}(a=1,2,3)$ providing the longitudinal degrees of freedom of the $W^{ \pm}$and $Z$ bosons are introduced by means of the field

$$
\Sigma(x)=e^{i \sigma^{a} \pi^{a}(x) / v}
$$

where $v \simeq 246 \mathrm{GeV}$ and $\sigma^{a}$ are the Pauli matrices. The field $\Sigma$ transforms linearly under $\mathrm{SU}(2)_{L} \times \mathrm{SU}(2)_{R}$. Introducing a scalar field $h$, assumed to transform as a singlet under the custodial symmetry, leads to the following effective Lagrangian [38]

$$
\begin{aligned}
\mathcal{L}= & \frac{1}{2}\left(\partial_{\mu} h\right)^{2}-V(h)+\frac{v^{2}}{4} \operatorname{Tr}\left[\left(D_{\mu} \Sigma\right)^{\dagger} D^{\mu} \Sigma\right]\left(1+2 a \frac{h}{v}+b \frac{h^{2}}{v^{2}}+b_{3} \frac{h^{3}}{v^{3}}+\cdots\right) \\
& -\frac{v}{\sqrt{2}}\left(\bar{u}_{L}^{i} \bar{d}_{L}^{i}\right) \Sigma\left[1+c \frac{h}{v}+c_{2} \frac{h^{2}}{v^{2}}+\cdots\right]\left(\begin{array}{l}
y_{i j}^{u} u_{R}^{j} \\
y_{i j}^{d} d_{R}^{j}
\end{array}\right)+\text { h.c. }+\mathcal{L}^{(4)}, \text { with } \\
V(h)= & \frac{1}{2} m_{h}^{2} h^{2}+d_{3}\left(\frac{m_{h}^{2}}{2 v}\right) h^{3}+d_{4}\left(\frac{m_{h}^{2}}{8 v^{2}}\right) h^{4}+\cdots, \\
\mathcal{L}^{(4)}= & \frac{g_{s}^{2}}{48 \pi^{2}} G^{\mu \nu a} G_{\mu \nu}^{a}\left(k_{g} \frac{h}{v}+\frac{1}{2} k_{2 g} \frac{h^{2}}{v^{2}}+\ldots\right)+\frac{e^{2}}{32 \pi^{2}} F_{\mu \nu} F^{\mu \nu}\left(k_{\gamma} \frac{h}{v}+\ldots\right),
\end{aligned}
$$

with the mass of the scalar given by $m_{h}$. In eq. (2.6) we have introduced the higherdimensional couplings $k_{g}, k_{2 g}, k_{\gamma}$, which are mediated at loop level by strong sector resonances. The Higgs couplings to fermions, $c, c_{2}, \ldots$, are assumed to be flavor-diagonal, so that MFV is realized. In table 1 the values of the couplings in the effective Lagrangian eq. (2.6) are listed in the SILH approach and in the holographic Higgs model MCHM5 (for the latter, only Higgs nonlinearities are considered). The SM with an elementary Higgs boson corresponds to $a=b=c=d_{3}=d_{4}=1, c_{2}=b_{3}=k_{g}=k_{2 g}=k_{\gamma}=0$ and vanishing higher order terms in $h$.

\section{Applying the Higgs low-energy theorem}

In this section we discuss applications of the Higgs low-energy theorem [23-25] in composite models. The LET allows one to obtain the leading interactions of the Higgs boson with gluons and photons arising from loops of heavy particles. By heavy particles we mean here both SM states ( $W$ and top) and new states belonging to the composite sector. These couplings are needed in the computation of the cross sections of single and double 


\begin{tabular}{|ccc|}
\hline Parameters & SILH & MCHM5, pure Higgs nonlinearities \\
\hline$a$ & $1-\left(c_{H}-c_{r} / 2\right) \xi / 2$ & $\sqrt{1-\xi}$ \\
$b$ & $1+\left(c_{r}-2 c_{H}\right) \xi$ & $1-2 \xi$ \\
$b_{3}$ & $\left(c_{r}-2 c_{H}\right) 2 \xi / 3$ & $-\frac{4}{3} \xi \sqrt{1-\xi}$ \\
$c$ & $1-\left(c_{H} / 2+c_{y}\right) \xi$ & $\frac{1-2 \xi}{\sqrt{1-\xi}}$ \\
$c_{2}$ & $-\left(c_{H}+3 c_{y}+c_{r} / 4\right) \xi / 2$ & $-2 \xi$ \\
$d_{3}$ & $1+\left(c_{6}-c_{r} / 4-3 c_{H} / 2\right) \xi$ & $\frac{1-2 \xi}{\sqrt{1-\xi}}$ \\
$d_{4}$ & $1+\left(6 c_{6}-25 c_{H} / 3-11 c_{r} / 6\right) \xi$ & $\frac{1-28 \xi(1-\xi) / 3}{1-\xi}$ \\
$k_{g}=k_{2 g}$ & $3 c_{g}\left(y_{t}^{2} / g_{\rho}^{2}\right) \xi$ & 0 \\
$k_{\gamma}$ & $2 c_{\gamma}\left(g^{2} / g_{\rho}^{2}\right) \xi$ & 0 \\
\hline
\end{tabular}

Table 1. Values of the couplings of the effective Lagrangian eq. (2.6) in the SILH framework (with $c_{T}=0$ ) and for MCHM5 considering only Higgs nonlinearities (i.e. neglecting the effects of resonances). The latter are taken from ref. [45, 46]. The values of the SILH parameters in MCHM5 are, in the 'natural' basis for the nonlinear $\sigma$-model where $c_{r}=-4 c_{H}, c_{H}=1 / 3, c_{r}=-4 / 3, c_{y}=$ $4 / 3, c_{6}=-4 / 3$.

Higgs production via gluon fusion at the LHC as well as of the partial width of the decay $h \rightarrow \gamma \gamma$. We will adopt a model-independent approach and compute these quantities in terms of the parameters of the effective Lagrangians defined in section 2, eqs. (2.1) and (2.6), putting special emphasis on the former, namely the SILH description. Our analysis extends the results of refs. [28, 47] to Higgs pair production in gluon fusion, and also includes a discussion of corrections to the LET approximation arising from higher order terms in the $1 / M$ expansion, where $M$ is the mass of the generic heavy particle running in the loops. Notice that the LET can be extended to 2-loop order to include the leading QCD corrections, see for example ref. [25] for applications in the SM. However, our discussion will be mainly limited to couplings at the leading 1-loop order.

\subsection{Higgs interactions with gluons}

According to the LET the interactions of the physical Higgs boson with gluons, mediated by loops of heavy coloured particles, can be obtained by treating the Higgs $H$ as a background field and taking the field-dependent mass of each heavy particle as a threshold for the running of the QCD gauge coupling. ${ }^{1}$ Assuming the heavy particles to transform in the fundamental representation of $\mathrm{SU}(3)_{c}$ one obtains the following effective Lagrangian

$$
\mathcal{L}_{\mathrm{eff}}=\frac{g_{s}^{2}}{64 \pi^{2}} G_{\mu \nu}^{a} G^{a \mu \nu} \sum_{p_{i}} \delta b_{p_{i}} \log m_{p_{i}}^{2}(H)
$$

\footnotetext{
${ }^{1}$ Throughout the paper, we will denote by $H$ both the Higgs doublet and the scalar field with $\langle H\rangle \neq 0$, as it will always be clear from the context which one we are referring to. On the other hand, $h$ denotes the physical Higgs scalar.
} 
where $\delta b=2 / 3$ if particle $p_{i}$ is a Dirac fermion, and $\delta b=1 / 6$ if it is a complex scalar. In this paper we will focus only on the effects of the heavy fermion sector, which in composite Higgs models typically includes new states beyond the top quark. By expanding the fielddependent masses of the heavy particles around the vacuum expectation value (VEV) $\langle H\rangle$ we obtain the couplings of the Higgs boson to gluons mediated by loops of heavy fermions

$$
\mathcal{L}_{h^{n} g g}=\frac{g_{s}^{2}}{96 \pi^{2}} G_{\mu \nu}^{a} G^{a \mu \nu}\left(A_{1} h+\frac{1}{2} A_{2} h^{2}+\ldots\right)
$$

where we have defined

$$
A_{n} \equiv\left(\frac{\partial^{n}}{\partial H^{n}} \log \operatorname{det} \mathcal{M}^{2}(H)\right)_{\langle H\rangle}
$$

with $\mathcal{M}^{2} \equiv \mathcal{M}^{\dagger} \mathcal{M}$, and $\mathcal{M}$ is the heavy fermion mass matrix. In the $\mathrm{SM}$ only the top quark contributes ${ }^{2}$ with $m_{t}(H)=y_{t} H / \sqrt{2}$, so that eq. (3.2) can be rewritten at all orders in $h$ as (see for example refs. [25, 48])

$$
\mathcal{L}_{h^{n} g g}=\frac{g_{s}^{2}}{48 \pi^{2}} G_{\mu \nu}^{a} G^{a \mu \nu} \log \left(1+\frac{h}{v}\right) .
$$

The corresponding gauge invariant operator is $\log \left(H^{\dagger} H\right) G_{\mu \nu}^{a} G^{a \mu \nu}$, which is associated with a chiral fermion. The lowest-order operator arising from vector-like fermions is instead $H^{\dagger} H G_{\mu \nu}^{a} G^{a \mu \nu}$. The effects of these two operators on double Higgs production were discussed in ref. [49].

Using eq. (3.2) it is straightforward to derive the expression of the hgg and hhgg couplings in the SILH formalism. We refer the reader to appendix A for a derivation, and simply report here the results. We remark that from now on we will work in the unitary gauge, where the Higgs doublet reads $(0, H / \sqrt{2})^{T}$. The effective coupling of the Higgs boson to two gluons reads (see appendix $\mathrm{A}$ )

$$
\mathcal{L}_{h g g}=\frac{g_{s}^{2}}{48 \pi^{2}} G_{\mu \nu}^{a} G^{a \mu \nu} \frac{h}{v}\left[\frac{1}{2}\left(\frac{\partial}{\partial \log H} \log \operatorname{det} \mathcal{M}^{2}(H)\right)_{H=v}-\frac{c_{H}}{2} \xi\right] .
$$

This coupling governs the rate of single Higgs production via gluon fusion, and its expression was already obtained in refs. [28, 47]. The production rate normalized to the SM one is given by the square of the expression in square brackets in eq. (3.5). On the other hand the effective coupling of two Higgs bosons to two gluons, which contributes to Higgs pair production via gluon fusion, has the following expression

$$
\mathcal{L}_{\text {hhgg }}=\frac{g_{s}^{2}}{96 \pi^{2}} G_{\mu \nu}^{a} G^{a} \mu \nu \frac{h^{2}}{v^{2}}\left[\frac{1}{2}\left(\left(\frac{\partial^{2}}{\partial(\log H)^{2}}-\frac{\partial}{\partial \log H}\right) \log \operatorname{det} \mathcal{M}^{2}(H)\right)_{H=v}-\frac{c_{r}}{4} \xi\right] .
$$

In terms of the effective Lagrangian in eq. (2.6), the couplings read

$$
\mathcal{L}_{h g g}=\frac{g_{s}^{2}}{48 \pi^{2}} G_{\mu \nu}^{a} G^{a \mu \nu} \frac{h}{v}\left(c+k_{g}\right), \quad \mathcal{L}_{h h g g}=\frac{g_{s}^{2}}{96 \pi^{2}} G_{\mu \nu}^{a} G^{a \mu \nu} \frac{h^{2}}{v^{2}}\left(2 c_{2}-c^{2}+k_{2 g}\right) .
$$

\footnotetext{
${ }^{2}$ The bottom contribution is non-negligible, but cannot be computed using the low-energy theorem, due to the smallness of the bottom quark mass.
} 
In the expression of the hhgg coupling in eq. (3.7), the first term comes from the triangle top loop involving the $t \bar{t} h h$ vertex, whereas the second is the contribution of top box diagrams, see figure 2. On the other hand, $k_{g}$ and $k_{2 g}$ are parameterizing the contributions from integrated-out heavy particles.

\subsection{Higgs interaction with photons}

Although the main focus of this paper is on gluon fusion, we give here the expression for the coupling of the Higgs boson to photons, as it is another loop process of crucial relevance for Higgs phenomenology at the LHC. This coupling receives contributions both from loops of heavy fermions and from the $W$ boson loop. Application of the LET leads to the following effective Lagrangian [25]

$$
\mathcal{L}_{\text {eff }}=\frac{e^{2}}{16 \pi^{2}} F_{\mu \nu} F^{\mu \nu}\left(\sum_{f} Q_{f}^{2} \log m_{f}^{2}(H)-\frac{7}{4} \log m_{W}^{2}(H)\right),
$$

which is valid for $m_{h} \lesssim 2 m_{W}, 2 m_{f}$, and where we have assumed that the heavy fermions transform in the fundamental representation of $\mathrm{SU}(3)_{c}$. Expanding around the VEV we obtain the $h \gamma \gamma$ interaction

$$
\mathcal{L}_{h \gamma \gamma}=\frac{e^{2}}{16 \pi^{2}} F_{\mu \nu} F^{\mu \nu} h\left(Q_{t}^{2} A_{1}-\frac{7}{4}\left(\frac{\partial}{\partial H} \log m_{W}^{2}(H)\right)_{\langle H\rangle}\right),
$$

where we have assumed that all fermions have electric charge equal to that of the top quark, ${ }^{3}$ $Q_{f}=Q_{t}=2 / 3$, and $A_{1}$ was defined in eq. (3.3). By performing simple manipulations we obtain (see appendix A)

$$
\begin{aligned}
\mathcal{L}_{h \gamma \gamma}=\frac{e^{2}}{32 \pi^{2}} F_{\mu \nu} F^{\mu \nu} \frac{h}{v}[ & 4 Q_{t}^{2}\left(\frac{1}{2}\left(\frac{\partial}{\partial \log H} \log \operatorname{det} \mathcal{M}^{2}(H)\right)_{H=v}-\frac{c_{H}}{2} \xi\right) \\
& \left.-J_{\gamma}\left(4 m_{W}^{2} / m_{h}^{2}\right)\left(1+\xi\left(\frac{c_{r}}{4}-\frac{c_{H}}{2}\right)\right)\right]
\end{aligned}
$$

where we have replaced the LET approximation for the $W$ loop with the full result encoded by the function

$$
J_{\gamma}(x)=F_{1}(x), \quad F_{1}(x)=2+3 x[1+(2-x) f(x)], \quad f(x)=\arcsin ^{2}\left(x^{-1 / 2}\right),
$$

which tends for large $x$ to $J_{\gamma}(\infty)=7=22 / 3-1 / 3$, where the first term comes from the transverse polarizations of the $W$ and is precisely equal to the gauge contribution to the $\beta$ function of the $\mathrm{SU}(2)_{L}$ coupling, while the second term arises from the eaten Goldstone bosons. The use of the full expression for the $W$ loop implies that the formal validity of eq. (3.10) is extended to $m_{h} \lesssim 2 m_{f}$. The rescaling of the decay width

\footnotetext{
${ }^{3}$ In all models considered in this paper, only top-like resonances contribute to the $h \gamma \gamma$ coupling. The extension to heavy states with different electric charge is straightforward.
} 


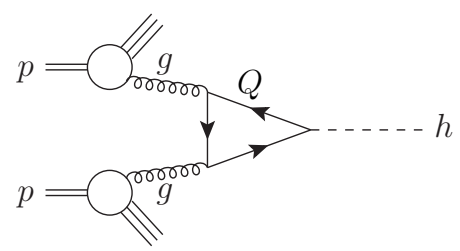

Figure 1. Generic diagram contributing to Higgs production in gluon fusion. In the SM case we have $Q \equiv t, b$. In composite Higgs models with additional heavy fermionic resonances these add to the particles $Q$ running in the loop.

$\Gamma(h \rightarrow \gamma \gamma) / \Gamma(h \rightarrow \gamma \gamma)_{\mathrm{SM}}$ is obtained by comparing the square of the expression multiplying $h F^{\mu \nu} F_{\mu \nu}$ in eq. (3.10) in the two cases. In terms of the parameters of the effective Lagrangian in eq. (2.6) the coupling reads

$$
\mathcal{L}_{h \gamma \gamma}=\frac{e^{2}}{32 \pi^{2}} F^{\mu \nu} F_{\mu \nu} \frac{h}{v}\left(4 Q_{t}^{2} c+k_{\gamma}-a J_{\gamma}\left(4 m_{W}^{2} / m_{h}^{2}\right)\right) .
$$

\subsection{Single Higgs production via gluon fusion}

For a SM Higgs boson, the gluon fusion process [50] gives the dominant production cross section at the LHC, see refs. [51, 52] for reviews. At leading order (LO), the process proceeds via a top loop, with a subleading contribution from the bottom loop, see figure 1 . In composite Higgs models extra heavy, colored fermions with sizeable couplings to the Higgs boson are typically present, whose contributions to the gluon fusion process should in general be taken into account. It has been shown $[27-29,53]$ that in explicit constructions the $g g \rightarrow h$ cross section (computed in the LET approximation) is insensitive to the details of the heavy fermion spectrum, i.e. it does not depend on the couplings and masses of composites, but only on the ratio $v / f$, where $f$ is the overall scale of the strong sector. This was found to be true both in models with partial compositeness and in Little Higgs theories. In fact, although the top Yukawa coupling receives a correction due to the mixing with resonances which depends on composite couplings, this contribution is exactly canceled by the loops of extra fermions, leading to a dependence of the $g g \rightarrow h$ rate only on $v / f$. This also implies that the cross section can be obtained by simply multiplying the SM one by $c^{2}$, where $c$ is the rescaling of the top Yukawa coming only from the nonlinearity of the $\sigma$-model, and neglecting corrections due to fermionic resonances.

Let us review how this cancellation arises. It is due to the fact that the determinant of the heavy fermion mass matrix takes the form

$$
\operatorname{det} \mathcal{M}^{2}(H)=F(H / f) \times P\left(\lambda_{i}, M_{i}, f\right),
$$

where $F$ is a function satisfying $F(0)=0$ since the top becomes massless in the limit of unbroken electroweak symmetry, and $P$ is a function of the composite couplings $\lambda_{i}$ and masses $M_{i}$, but independent of $H$. It is then immediate to see that the $h g g$ coupling in eq. (3.5) does not depend on the masses and couplings of the resonances. ${ }^{4}$ The origin

\footnotetext{
${ }^{4}$ The coefficient $c_{H}$ does not receive contributions from the heavy fermion sector. It is, however, generated by integrating out heavy scalars or vectors [47], see appendix B.1 for an explicit example.
} 
of the factorization in eq. (3.13) was explained in the context of partial compositeness in ref. [29], by means of a spurion analysis. There it was also pointed out that such a factorization can break down if the top mixes with more than one composite operator, leading to a dependence of the $h g g$ vertex on composite couplings. Nevertheless in many explicit constructions, including Little Higgs models, the factorization in eq. (3.13) takes place. Still, the independence of the $h g g$ vertex on the composite couplings (collectively denoted by $\lambda_{i}$ ) holds exactly only in the LET approximation, and corrections due to finite fermion mass effects are expected. We can estimate the residual dependence on the $\lambda_{i}$ due to finite fermion mass effects in a simple way. Assuming for simplicity the presence of only one top partner $T$, the mass eigenvalues can be written at $\mathcal{O}\left(1 / f^{2}\right)$ as

$$
m_{t}(H)=\frac{y_{t} H}{\sqrt{2}}\left(1-\frac{c_{y}^{(t)}}{2} \frac{H^{2}}{f^{2}}\right), \quad m_{T}(H)=\lambda_{T} f\left(1+a_{T} \frac{H^{2}}{f^{2}}\right),
$$

where $a_{T}$ is a parameter dependent on the couplings $\lambda_{i}=\left\{y_{t}, \lambda_{T}\right\}$ as $a_{T}=\mathcal{O}\left(y_{t}^{2} / \lambda_{T}^{2}\right) .{ }^{5}$ On the other hand, we can write $c_{y}^{(t)}=c_{y}^{(\sigma)}+\mathcal{O}\left(y_{t}^{2} / \lambda_{T}^{2}\right)$, where $c_{y}^{(\sigma)}$ is a constant arising from the pure nonlinearity of the $\sigma$-model. The LET result for the $h g g$ coupling reads, taking the effect of $c_{H}$ into account,

$$
\mathcal{L}_{h g g}=\frac{g_{s}^{2}}{48 \pi^{2}} G_{\mu \nu}^{a} G^{a} \mu \nu \frac{h}{v}\left(1-\left(c_{y}^{(t)}-2 a_{T}+\frac{c_{H}}{2}\right) \xi\right) .
$$

Notice that in the limit where $T$ is heavy, corresponding to large $\lambda_{T}$, the effects of the heavy resonance on the $h g g$ coupling vanish. In fact, $a_{T}$ goes to zero, whereas $c_{y}^{(t)} \rightarrow c_{y}^{(\sigma)}$, implying that only the nonlinearity in the top Yukawa arising from the nonlinear $\sigma$-model is relevant.

By using the expression of the top Yukawa coupling $\left(m_{t} / v\right)\left(1-\left(c_{y}^{(t)}+c_{H} / 2\right) \xi\right)$ we can compute explicitly the top loop diagram, retaining the first subleading term in the $1 / m_{t}^{2}$ expansion. This is the leading correction to the LET coupling, given that $m_{T} \gg m_{t}$. Thus eq. (3.15) is improved to

$$
\mathcal{L}_{h g g}=\frac{g_{s}^{2}}{48 \pi^{2}} G_{\mu \nu}^{a} G^{a \mu \nu} \frac{h}{v}\left[1-\left(c_{y}^{(t)}-2 a_{T}+\frac{c_{H}}{2}\right) \xi+\frac{7}{120} \frac{m_{h}^{2}}{m_{t}^{2}}\left(1-\xi\left(c_{y}^{(t)}+c_{H} / 2\right)\right)+\ldots\right],
$$

where we have used $\hat{s}=m_{h}^{2}$, and the ellipses stand for subleading corrections (including terms of order $1 / m_{T}^{2}$ ). The independence of the LET $h g g$ vertex of the composite couplings $\lambda_{i}$ is equivalent to the statement that $c_{y}^{(t)}-2 a_{T}$ is a constant, $c_{y}^{(t)}-2 a_{T}=c_{y}^{(\sigma)}{ }^{6}$ If this is the case then the dependence on the $\lambda_{i}$ of eq. (3.16) is due to the last term, and we can estimate the sensitivity of the cross section to the $\lambda_{i}$ to be, for a light top partner $\lambda_{T} \sim y_{t}$,

$$
\frac{\delta \sigma(g g \rightarrow h)}{\sigma(g g \rightarrow h)_{\mathrm{SM}}} \sim \frac{7}{60} \frac{m_{h}^{2}}{m_{t}^{2}} \xi \simeq 0.06 \xi
$$

\footnotetext{
${ }^{5}$ If the quadratic divergence in the Higgs mass due to the top is cancelled by $T$, then the absence of an $\mathcal{O}\left(H^{2}\right)$ term in $\operatorname{Tr} \mathcal{M}^{2}(H)=m_{t}^{2}(H)+m_{T}^{2}(H)$ implies $a_{T}=-y_{t}^{2} /\left(4 \lambda_{T}^{2}\right)$. See for example the explicit values of $c_{y}^{(t)}$ and $a_{T}$ in the Littlest Higgs model, reported in appendix B.1, eq. (B.18).

${ }^{6}$ Notice that by using eq. (3.14) one finds $\operatorname{det} \mathcal{M}^{2}=y_{t}^{2} \lambda_{T}^{2} f^{2} H^{2}\left(1-\left(c_{y}^{(t)}-2 a_{T}\right) H^{2} / f^{2}\right) / 2$. So if the factorization in eq. (3.13) holds then $c_{y}^{(t)}-2 a_{T}$ is a constant.
} 

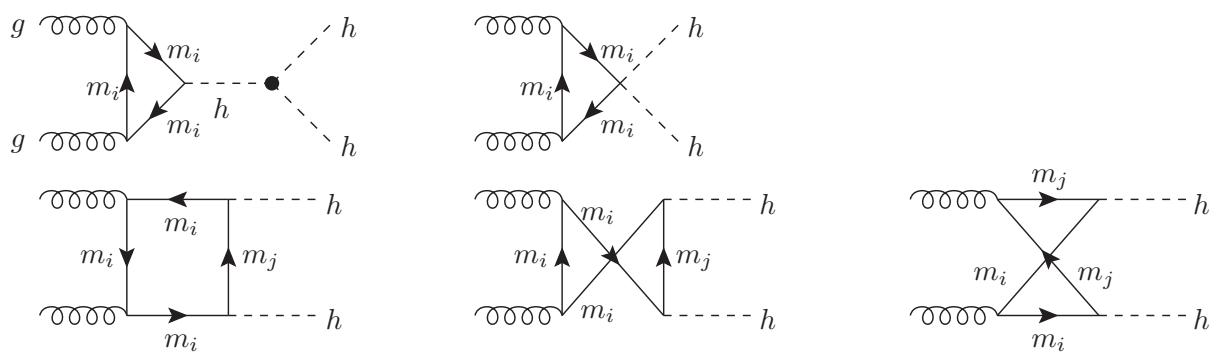

Figure 2. Generic diagrams contributing to double Higgs production via gluon fusion in composite Higgs models with $n_{f}$ novel fermionic resonances of mass $m_{i}\left(i=1, \ldots, n_{f}\right)$. The index $j$ is introduced to indicate that different fermions can contribute to each box diagram.

where in the last equality we assumed $m_{h}=125 \mathrm{GeV}$. Thus corrections are expected to be very small even for large $\xi$. This estimate will be confirmed in section 5 , where the $g g \rightarrow h$ cross section will be computed in MCHM5 retaining the full mass dependence. We note that in this model the cross section can be strongly suppressed compared to the $\mathrm{SM}$, reaching $\sigma / \sigma_{\mathrm{SM}}=1 / 3$ for a low compositeness scale $\xi=0.25$. However, the Higgs branching ratios into $\gamma \gamma, W W$ and $Z Z$ are enhanced compared to the SM, so MCHM5 can still be compatible with the excesses observed by ATLAS and CMS at $m_{h} \sim 125 \mathrm{GeV}$ even for values of $\xi$ as large as those considered in this paper $[45,46]$.

\subsection{Double Higgs production via gluon fusion}

Within the SM, double Higgs production via gluon fusion received interest mainly because it is sensitive to the trilinear Higgs self-coupling [54-57], see the first diagram in figure 2. In composite Higgs models, the process $g g \rightarrow h h$ is affected essentially in two main ways. First, the nonlinearity of the strong sector gives rise to a $f \bar{f} h h$ coupling (which vanishes in the SM) and thus to a genuinely new contribution to the amplitude, see the second diagram in figure 2. Second, one should take into account the effects of top partners, which include also new box diagrams involving off-diagonal Yukawa couplings ${ }^{7}$ (shown in the second line of figure 2). A first study of $g g \rightarrow h h$ in composite Higgs models, neglecting top partners, was performed in ref. [36], where it was found that a large enhancement of the cross section is possible due to the new $t \bar{t} h h$ coupling (see also ref. [35] for an earlier study in the context of Little Higgs models). For example, in MCHM5 with $\xi=0.25$, which corresponds to $f \simeq 500 \mathrm{GeV}$, the cross section was found to be about 3.6 times larger than in the SM. Recently, ref. [37] performed a model-independent study of the process, making reference to the effective Lagrangian in eq. (2.6) and again neglecting the effects of top partners, and found a large sensitivity of the cross section to the $c_{2}$ coefficient parameterizing the $t \bar{t} h h$ coupling.

In this paper we include for the first time the effects of top partners in double Higgs production via gluon fusion. This is especially interesting in the light of the results of refs. [10-13], where a naturally light composite Higgs was shown to be tightly correlated

\footnotetext{
${ }^{7}$ Note that these Yukawa couplings only involve the top quark and its charge $2 / 3$ heavy composite partners.
} 
with the presence of light top partners, as such light resonances can in principle affect the $g g \rightarrow h h$ cross section in a sizeable way. Our analysis will confirm that this is indeed the case.

We start by discussing the cross section in the LET approximation, which greatly simplifies the computation. In this limit, the amplitude is simply the sum of two diagrams, one with the effective $h g g$ coupling followed by a trilinear Higgs coupling and the other involving the effective hhgg coupling. Adopting the SILH formalism, and recalling the expressions of the relevant Feynman rules

$$
\begin{aligned}
h g g & : i \frac{\alpha_{s}}{3 \pi v} \delta^{a b}\left(p_{1}^{\nu} p_{2}^{\mu}-p_{1} \cdot p_{2} g^{\mu \nu}\right)\left[\frac{1}{2}\left(\frac{\partial}{\partial \log H} \log \operatorname{det} \mathcal{M}^{2}(H)\right)_{H=v}-\frac{c_{H}}{2} \xi\right], \\
h h g g & : i \frac{\alpha_{s}}{3 \pi v^{2}} \delta^{a b}\left(p_{1}^{\nu} p_{2}^{\mu}-p_{1} \cdot p_{2} g^{\mu \nu}\right)\left[\frac{1}{2}\left(\left(\frac{\partial^{2}}{\partial(\log H)^{2}}-\frac{\partial}{\partial \log H}\right) \log \operatorname{det} \mathcal{M}^{2}(H)\right)_{H=v}-\frac{c_{r}}{4} \xi\right], \\
h h h & :-i 3 \frac{m_{h}^{2}}{v}\left[1+\xi\left(c_{6}-\frac{3}{2} c_{H}-\frac{1}{4} c_{r}\right)\right]
\end{aligned}
$$

(where $p_{1,2}$ denote the momenta of the incoming gluons), we can write the amplitude as

$$
\mathcal{A}_{\mathrm{LET}}(g g \rightarrow h h)=\frac{\alpha_{s}}{3 \pi v^{2}} \delta^{a b}\left(p_{1}^{\nu} p_{2}^{\mu}-p_{1} \cdot p_{2} g^{\mu \nu}\right) C_{\mathrm{LET}}(\hat{s}),
$$

where

$$
\begin{aligned}
C_{\mathrm{LET}}(\hat{s})=\frac{3 m_{h}^{2}}{\hat{s}-m_{h}^{2}}[ & \left.\frac{1}{2}\left(\frac{\partial}{\partial \log H} \log \operatorname{det} \mathcal{M}^{2}(H)\right)_{H=v}+\xi\left(c_{6}-2 c_{H}-\frac{c_{r}}{4}\right)\right] \\
& +\frac{1}{2}\left(\left(\frac{\partial^{2}}{\partial(\log H)^{2}}-\frac{\partial}{\partial \log H}\right) \log \operatorname{det} \mathcal{M}^{2}(H)\right)_{H=v}-\frac{c_{r}}{4} \xi \\
= & \frac{3 m_{h}^{2}}{\hat{s}-m_{h}^{2}}\left(1-\xi\left(c_{y}^{(t)}-c_{6}+2 c_{H}+\frac{c_{r}}{4}-3 c_{g} \frac{y_{t}^{2}}{g_{\rho}^{2}}\right)\right)-\left(1+\xi\left(c_{y}^{(t)}+\frac{c_{r}}{4}-3 c_{g} \frac{y_{t}^{2}}{g_{\rho}^{2}}\right)\right),
\end{aligned}
$$

with $\hat{s} \equiv\left(p_{1}+p_{2}\right)^{2}$ denoting the partonic center-of-mass (c.m.) energy. To obtain the second equality in eq. (3.20) we used eqs. (A.3) and (A.4) contained in appendix A. It is immediate to check that the combinations $c_{y}^{(t)}-c_{6}+2 c_{H}+c_{r} / 4$ and $c_{y}^{(t)}+c_{r} / 4$ are invariant under the reparameterization in eqs. (2.2) and (2.3). For completeness, we also give the result in terms of the coefficients of the effective Lagrangian in eq. (2.6):

$$
C_{\mathrm{LET}}^{\mathcal{L}_{\mathrm{eff}}}(\hat{s})=\frac{3 m_{h}^{2}}{\hat{s}-m_{h}^{2}}\left(c+k_{g}\right) d_{3}+2 c_{2}-c^{2}+k_{2 g} .
$$

The partonic cross section reads

$$
\hat{\sigma}_{g g \rightarrow h h}=\frac{G_{F}^{2} \alpha_{s}^{2}(\mu) \hat{s}}{128(2 \pi)^{3}} \frac{1}{9} \sqrt{1-\frac{4 m_{h}^{2}}{\hat{s}}} C_{\mathrm{LET}}^{2}(\hat{s}) .
$$

The hadronic cross section is obtained by convolution with the parton distribution function $f_{g / P}$ of the gluon in the proton,

$$
\sigma=\int_{4 m_{h}^{2} / s}^{1} d \tau \int_{\tau}^{1} \frac{d x}{x} f_{g / P}(x, Q) f_{g / P}(\tau / x, Q) \hat{\sigma}_{g g \rightarrow h h}(\tau s),
$$



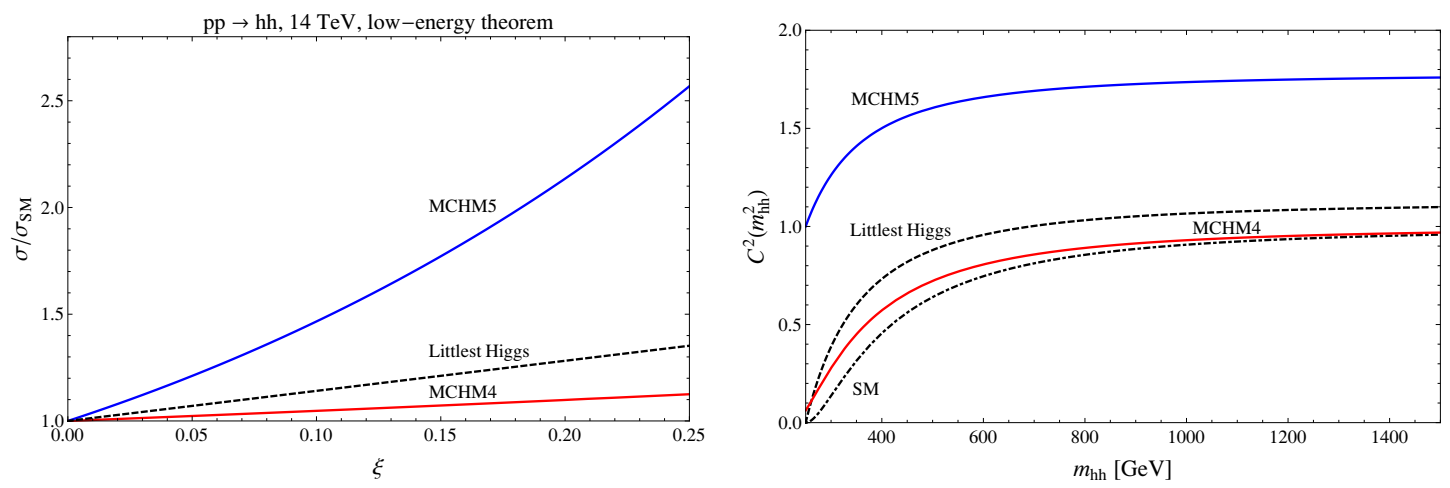

Figure 3. (Left panel) The $p p \rightarrow h h$ cross section for $m_{h}=125 \mathrm{GeV}$ at LHC14, computed using the LET, normalized to the SM cross section also computed in the $m_{t} \rightarrow \infty$ limit. MHCM5 is discussed in detail in the text, whereas the $g g \rightarrow h h$ amplitudes for MCHM4 and for the Littlest Higgs model are given in appendix B. (Right panel) Square of the function $C\left(m_{h h}^{2}\right)$, which was defined in eq. (3.20) and is proportional to the LET $g g \rightarrow h h$ amplitude, in the three models under consideration (for $\xi=0.25$ ) and in the SM, as a function of $m_{h h}=\sqrt{\hat{s}}$.

with the collider c.m. energy $s$ related to $\hat{s}$ by $\hat{s}=\tau s$. The renormalization scale $\mu$ and the factorization scale $Q$ are chosen equal to the invariant mass of the Higgs boson pair, $\mu=Q=\sqrt{\hat{s}}$. Throughout the paper, the parton distribution functions of MSTW2008 [58] are employed. For $\xi \rightarrow 0$, eq. (3.20) correctly reproduces the SM result in the limit of large top mass $[32,33]$

$$
C_{\mathrm{LET}}^{\mathrm{SM}}(\hat{s})=\frac{3 m_{h}^{2}}{\hat{s}-m_{h}^{2}}-1
$$

In the SM the $m_{t} \rightarrow \infty$ limit gives a cross section in agreement with the full result only within $20 \%$ for $m_{h} \lesssim 200 \mathrm{GeV}$ (for $m_{h}=125 \mathrm{GeV}$ we find $\sigma_{\mathrm{LET}}^{\mathrm{SM}}=14.6 \mathrm{fb}$ and $\sigma_{\text {full }}^{\mathrm{SM}}=17.9 \mathrm{fb}$ ) and moreover it produces incorrect kinematic distributions, as noticed in ref. [34]. Thus we expect the LET to be in general less accurate in $g g \rightarrow h h$ than in single Higgs production.

From eq. (3.20) we read off that in models where the factorization eq. (3.13) of $\operatorname{det} \mathcal{M}^{2}$ holds, the $g g \rightarrow h h$ LET cross section is insensitive to composite couplings, due to a cancellation completely analogous to the one that we discussed for single Higgs production. In the left panel of figure 3 we show for $m_{h}=125 \mathrm{GeV}$ and a c.m. energy of $14 \mathrm{TeV}$ the $p p \rightarrow h h$ cross section normalized to the SM cross section (both were computed applying the LET) as a function of $\xi$ for some well-known models, in all of which the cancellation holds. We note that in MCHM5 the enhancement of the cross section is striking. This can be traced back to the behavior of the function $C_{\mathrm{LET}}(\hat{s})$, which is proportional to the LET amplitude and is shown in the right panel of figure 3 for the three models under consideration and for the SM. The enhancement for MCHM5 is evident. As pointed out for the first time in ref. [36], where the $g g \rightarrow h h$ process was studied in MCHM5 considering only Higgs nonlinearities (or equivalently in the limit of heavy top partners) but keeping the full dependence on $m_{t}$, the dramatic increase of the $g g \rightarrow h h$ cross section compared 
to the SM is mostly due to the presence of a new $t \bar{t} h h$ coupling. The large enhancement of $g g \rightarrow h h$ in MCHM5 is in contrast with the strong suppression in the same model of the single Higgs production cross section, which for $\xi=0.25$ equals $1 / 3$ of the SM value (see section 5).

By comparison with ref. [36] we find that when fermionic resonances are above the cutoff, the LET underestimates the ratio $\sigma^{\mathrm{MCHM} 5} / \sigma^{\mathrm{SM}}$ by about $30 \%$ : for example for $\xi=0.25$, application of the LET gives a cross section of 2.6 times the SM, whereas ref. [36] found an enhancement factor of 3.6. This difference is due to the fact that in the former case $m_{h} \ll m_{t}$ is assumed, whereas in the latter the full $m_{t}$ dependence was retained. Notice that the best estimate of the cross section that can be obtained using the LET is $\sigma^{\mathrm{MCHM}}=\left(\sigma_{\mathrm{LET}}^{\mathrm{MCHM} 5} / \sigma_{\mathrm{LET}}^{\mathrm{SM}}\right) \times \sigma_{\text {full }}^{\mathrm{SM}}$, because part of the corrections due to the finite top mass should cancel in the ratio of LET cross sections. In fact, in terms of cross sections the disagreement between the LET and the result obtained taking into account only Higgs nonlinearities is larger. For $\xi=0.25$ we obtain $\sigma_{\mathrm{LET}}^{\mathrm{MCHM} 5}=37 \mathrm{fb}$, whereas ref. [36] found $\sigma^{\mathrm{MCHM} 5}=64 \mathrm{fb}$, i.e. the difference is of order $50 \%$.

In order to understand this behavior we investigated in more detail the validity of the LET both for single and double Higgs production. In single production the expansion parameter is $m_{h}^{2} /\left(4 m_{t}^{2}\right)$ and the series converges very quickly. In double Higgs production on the other hand, one needs to expand in $\hat{s} /\left(4 m_{t}^{2}\right)$ with $\hat{s} \geq 4 m_{h}^{2}$, which is not small, so in general the expansion does not work as well as for the single Higgs case. In MCHM5, the validity of the expansion gets even worse. The reason why the LET is less accurate for MCHM5 than for the SM (where it underestimates the cross section by about 20\%) is mainly the presence of the new triangle diagram containing the $t \bar{t} h h$ coupling, which contrarily to the triangle diagram involving the virtual Higgs exchange does not vanish at large $\hat{s}$. This is confirmed by taking into account the corrections of $\mathcal{O}\left(1 / m_{t}^{2}\right)$ to the LET result, which are reported in appendix E.5. Compared to the SM we have an additional contribution $\sim \xi$ from the two-Higgs two-fermion coupling which goes like $\sim \hat{s} / m_{t}^{2}$, and which in contrast to the triangle diagram with virtual Higgs exchange is not suppressed by the Higgs propagator, see eqs. (E.20) and (E.21). Therefore in MCHM5 for large $\xi$, where the coupling $c_{2}$ is sizeable (see table 1 ), the corrections do not improve at all the LET result. For a model-independent study of the $g g \rightarrow h h$ process including only modifications to the top couplings, see ref. [37].

While in the LET approximation the contributions of loops of top partners to the $g g \rightarrow h h$ amplitude exactly cancel out with that coming from the modification of the top Yukawa due to mixing with resonances, the sensitivity to composite couplings of the full double Higgs production cross section (computed retaining all dependence on masses) is expected to be much larger than for $g g \rightarrow h$, where it was shown to be negligible. By direct computation in MCHM5, we will see in section 6 that this is the case, i.e. the full $g g \rightarrow h h$ cross section has a sizeable sensitivity to the details of the spectrum of the top partners. This effect is not captured by the simple LET result, which is completely determined by $\xi$. Therefore, while the low-energy theorem provides a useful tool to obtain a rough estimate of the cross section, a complete loop computation is needed to describe correctly the effects of top partners in the $g g \rightarrow h h$ process. 


\section{Composite Higgs model with extra fermionic resonances}

We consider a composite Higgs scenario with the symmetry group of the stronglyinteracting sector given by $\mathrm{SO}(5)$, which is spontaneously broken down to $\mathrm{SO}(4)$ at the scale $f$. In order to correctly reproduce the fermion charges an additional local symmetry $\mathrm{U}(1)_{X}$ is introduced, leading to the symmetry breaking pattern $\mathrm{SO}(5) \times \mathrm{U}(1)_{X} / \mathrm{SO}(4) \times \mathrm{U}(1)_{X}$. This is the minimal realisation including custodial symmetry and which implies four Goldstone bosons (GBs) transforming as a 4 of $\mathrm{SO}(4) \sim \mathrm{SU}(2)_{L} \times \mathrm{SU}(2)_{R}$. The $\mathrm{SM}$ electroweak group $\mathrm{SU}(2)_{L} \times \mathrm{U}(1)_{Y}$ is embedded into $\mathrm{SO}(4) \times \mathrm{U}(1)_{X}$ and the hypercharge $Y$ is then given by $Y=T_{R}^{3}+X[40,41]$. The GBs are parameterized in terms of the field

$$
\Sigma=\Sigma_{0} e^{\Pi / f}, \quad \Pi=-i \sqrt{2} T^{\hat{a}} h^{\hat{a}}, \quad \Sigma_{0}=(0,0,0,0,1),
$$

where $T^{\hat{a}}(\hat{a}=1, \ldots, 4)$ are the generators of $\mathrm{SO}(5) / \mathrm{SO}(4)$, and $h^{\hat{a}}$ are the 4 real GBs. Using the explicit expressions of the $\mathrm{SO}(4)$ and $\mathrm{SO}(5) / \mathrm{SO}(4)$ generators $(i, j=1, \ldots, 5)$

$$
\begin{aligned}
T_{i j}^{a_{L, R}} & =-\frac{i}{2}\left[\frac{1}{2} \epsilon^{a b c}\left(\delta_{i}^{b} \delta_{j}^{c}-\delta_{j}^{b} \delta_{i}^{c}\right) \pm\left(\delta_{i}^{a} \delta_{j}^{4}-\delta_{j}^{a} \delta_{i}^{4}\right)\right], \quad a_{L, R}=1,2,3, a, b, c=1,2,3 \\
T_{i j}^{\hat{a}} & =-\frac{i}{\sqrt{2}}\left(\delta_{i}^{\hat{a}} \delta_{j}^{5}-\delta_{j}^{\hat{a}} \delta_{i}^{5}\right), \quad \hat{a}=1, \ldots, 4
\end{aligned}
$$

one obtains

$$
\Sigma=\frac{\sin (h / f)}{h}\left(h_{1}, h_{2}, h_{3}, h_{4}, h \operatorname{cotan}(h / f)\right), \quad h=\sqrt{\sum_{\hat{a}} h_{\hat{a}}^{2}} .
$$

The two-derivative Lagrangian reads

$$
\mathcal{L}_{\text {kin }}=\frac{f^{2}}{2}\left(D_{\mu} \Sigma\right)\left(D^{\mu} \Sigma\right)^{T}, \quad D_{\mu} \Sigma=\partial_{\mu} \Sigma+i g W_{\mu}^{a} \Sigma T_{L}^{a}+i g^{\prime} B_{\mu} \Sigma T_{R}^{3} .
$$

By performing an $\mathrm{SO}(4)$ rotation, it is always possible to align the Higgs VEV to the $h_{3}$ direction, thus identifying $H=h_{3}$, where $H$ is the Higgs field (with $\langle H\rangle \neq 0$ ). Then in the unitary gauge

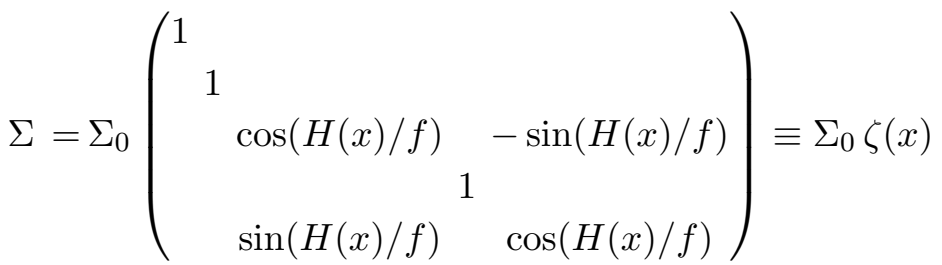

$$
\begin{aligned}
& =(0,0, \sin (H / f), 0, \cos (H / f)) \text {, }
\end{aligned}
$$

and therefore

$$
\mathcal{L}_{\text {kin }}=\frac{1}{2} \partial_{\mu} H \partial^{\mu} H+\frac{g^{2} f^{2}}{4} \sin ^{2}\left(\frac{H}{f}\right)\left[W_{\mu}^{+} W^{-\mu}+\frac{1}{2 \cos ^{2} \theta_{W}} Z_{\mu} Z^{\mu}\right]
$$

which fixes $f^{2} \sin ^{2}(\langle H\rangle / f)=v^{2} \simeq(246 \mathrm{GeV})^{2}$.

Fermionic resonances are described using the language of partial compositeness. We introduce vector-like fermions which have quantum numbers such that they can mix linearly 


\begin{tabular}{|c|c|c|c|c|}
\hline field & $T_{L}^{3}$ & $T_{R}^{3}$ & $Y$ & $Q_{e l}=T_{L}^{3}+Y$ \\
\hline$T$ & $+1 / 2$ & $-1 / 2$ & $1 / 6$ & $+2 / 3$ \\
$B$ & $-1 / 2$ & $-1 / 2$ & $1 / 6$ & $-1 / 3$ \\
$X^{5 / 3}$ & $+1 / 2$ & $+1 / 2$ & $7 / 6$ & $+5 / 3$ \\
$X^{2 / 3}$ & $-1 / 2$ & $+1 / 2$ & $7 / 6$ & $+2 / 3$ \\
$\tilde{T}$ & 0 & 0 & $2 / 3$ & $+2 / 3$ \\
\hline
\end{tabular}

Table 2. SM quantum numbers of the composite fermions in $\psi$. The last column denotes the electric charge.

with the SM fermions $q_{L}=\left(t_{L}, b_{L}\right)^{T}$ and $t_{R}$, and which at the same time have 'protoYukawa' interactions with the composite Higgs. We introduce composite fermions transforming as a complete $\mathbf{5}_{2 / 3}$ under $\mathrm{SO}(5) \times \mathrm{U}(1)_{X}$. This representation has the phenomenologically desirable feature that no tree-level corrections to the $Z-b-\bar{b}$ coupling arise, provided a discrete symmetry $P_{L R}$ exchanging the $\mathrm{SU}(2)_{L}$ and $\mathrm{SU}(2)_{R}$ factors is enforced [41, 59-61]. The composite multiplets can be written as

$$
\psi=\frac{1}{\sqrt{2}}\left(\begin{array}{c}
B-X^{5 / 3} \\
-i\left(B+X^{5 / 3}\right) \\
T+X^{2 / 3} \\
i\left(T-X^{2 / 3}\right) \\
\sqrt{2} \tilde{T}
\end{array}\right)
$$

Under $\mathrm{SU}(2)_{L} \times \mathrm{SU}(2)_{R}$, a $\mathbf{5}$ of $\mathrm{SO}(5)$ decomposes as $\mathbf{5} \sim(\mathbf{2}, \mathbf{2}) \oplus(\mathbf{1}, \mathbf{1})$. The $\mathrm{SU}(2)_{L}$ doublets $Q=(T, B)^{T}$ and $X=\left(X^{5 / 3}, X^{2 / 3}\right)^{T}$ form a bidoublet $(\mathbf{2}, \mathbf{2})$ under $\operatorname{SU}(2)_{L} \times$ $\mathrm{SU}(2)_{R}$, while $\tilde{T}$ is a singlet $(\mathbf{1}, \mathbf{1})$. The SM quantum numbers of the composite fields are summarized in table 2 . Note that $Q$ has the same quantum numbers as the elementary doublet $q_{L}=\left(t_{L}, b_{L}\right)^{T}$, whereas $\tilde{T}$ has the same quantum numbers as $t_{R}$. The doublet $X$ is peculiar of the $\mathbf{5}$ representation (it is absent in the most minimal case of the spinorial representation 4). Taking into account only one set of fermionic composites, the Lagrangian for the fermion sector then reads

$$
\begin{aligned}
\mathcal{L}_{f}= & i \bar{q}_{L} \not D q_{L}+i \bar{t}_{R} \not D t_{R}+i \bar{b}_{R} \not D b_{R}+i \bar{\psi}_{L} \not D \psi_{L}+i \bar{\psi}_{R} \not D \psi_{R} \\
& -y f\left(\bar{\psi}_{L} \Sigma^{T}\right)\left(\Sigma \psi_{R}\right)-M_{0} \bar{\psi}_{L} \psi_{R}+\text { h.c. } \\
& -\Delta_{L} \bar{q}_{L} Q_{R}-\Delta_{R} \overline{\tilde{T}}_{L} t_{R}+\text { h.c. }
\end{aligned}
$$

where the covariant derivative acting on $\psi$ is given by

$$
D_{\mu} \psi=\left[\partial_{\mu}-i g W_{\mu}^{a} T_{L}^{a}-i g^{\prime} B_{\mu}\left(T_{R}^{3}+X\right)\right] \psi, \quad X=(2 / 3) \mathbb{1}_{5} .
$$

For later convenience, we also give the embedding of $q_{L}$ and $t_{R}$ in $\mathrm{SO}(5)$ vectors:

$$
\mathcal{Q}_{L}=\frac{1}{\sqrt{2}}\left(\begin{array}{lll}
b_{L}-i b_{L} t_{L} i t_{L} & 0
\end{array}\right)^{T}, \quad \mathcal{T}_{R}=\frac{1}{\sqrt{2}}\left(\begin{array}{lllll}
0 & 0 & 0 & 0 & \sqrt{2} t_{R}
\end{array}\right)^{T} .
$$


Using these expressions the linear mixings can be rewritten as

$$
\mathcal{L}_{\text {mix }}=-\Delta_{L} \overline{\mathcal{Q}}_{L} \psi_{R}-\Delta_{R} \bar{\psi}_{L} \mathcal{T}_{R}+\text { h.c. }
$$

From the Lagrangian in eq. (4.10) we obtain the mass terms and the Yukawa couplings. The mass matrix reads

$$
-\mathcal{L}_{m}=\left(\begin{array}{c}
t_{L} \\
T_{L} \\
X_{L}^{2 / 3} \\
\tilde{T}_{L}
\end{array}\right)\left(\begin{array}{cccc}
0 & \Delta_{L} & 0 & 0 \\
0 & M_{0}+\frac{f y s^{2}}{2} & \frac{y f s^{2}}{2} & \frac{y f s c}{\sqrt{2}} \\
0 & \frac{y f s^{2}}{2} & M_{0}+\frac{f y s^{2}}{2} & \frac{y f s c}{\sqrt{2}} \\
\Delta_{R} & \frac{y f s c}{\sqrt{2}} & \frac{y f s c}{\sqrt{2}} & M_{0}+y f c^{2}
\end{array}\right)\left(\begin{array}{c}
t_{R} \\
T_{R} \\
X_{R}^{2 / 3} \\
\tilde{T}_{R}
\end{array}\right)+\text { h.c. }
$$

where we have introduced the abbreviation $s \equiv \sin (\langle H\rangle / f)=v / f$ and analogously $c \equiv \cos (\langle H\rangle / f)$. The diagonalization of the matrix, which mixes fundamental fields and composite states, is immediate before electroweak symmetry breaking, i.e. for $v=0$. Then the mass terms become diagonal after the rotations

$$
\begin{aligned}
& \left(\begin{array}{c}
q_{L} \\
Q_{L}
\end{array}\right) \rightarrow\left(\begin{array}{cc}
\cos \phi_{L} & \sin \phi_{L} \\
-\sin \phi_{L} & \cos \phi_{L}
\end{array}\right)\left(\begin{array}{c}
q_{L} \\
Q_{L}
\end{array}\right), \quad \tan \phi_{L}=\frac{\Delta_{L}}{M_{0}} \\
& \left(\begin{array}{c}
t_{R} \\
\tilde{T}_{R}
\end{array}\right) \rightarrow\left(\begin{array}{cc}
\cos \phi_{R} & \sin \phi_{R} \\
-\sin \phi_{R} & \cos \phi_{R}
\end{array}\right)\left(\begin{array}{c}
t_{R} \\
\tilde{T}_{R}
\end{array}\right), \quad \tan \phi_{R}=\frac{\Delta_{R}}{M_{0}+y f}
\end{aligned}
$$

In this limit the top is massless, whereas the masses of the composite states are

$$
M_{Q}=\frac{M_{0}}{c_{L}}, \quad M_{X}=M_{0}, \quad M_{\tilde{T}}=\frac{y f+M_{0}}{c_{R}} .
$$

Electroweak symmetry breaking effects generate additional mixings, which also involve $t_{L}$ and $t_{R}$. Thus the top becomes massive due to its mixing with composite states. At the leading order in $\xi \equiv v^{2} / f^{2}$ we have

$$
m_{t}=y \sin \phi_{L} \sin \phi_{R} \frac{v}{\sqrt{2}}
$$

Furthermore, the masses of the composite fermions in eq. (4.16) get corrections of order $\xi$. The Lagrangian eq. (4.10), however, does not give rise to a mass for the bottom quark, because there is no composite in $\psi$ that has the right quantum numbers to mix with $b_{R}$. Rather than introducing another fermionic multiplet (e.g., a $\mathbf{5}_{-1 / 3}$ ) to solve this issue, we introduce a small breaking of the partial compositeness pattern, namely a Yukawa coupling of the Higgs to elementary states

$$
\mathcal{L}_{b}=-\lambda_{b} \bar{q}_{L} H b_{R}+\text { h.c. },
$$

where $H$ is the Higgs doublet. We will, however, neglect the small effects proportional to $\lambda_{b}$.

Expanding the proto-Yukawa term up to second order in the physical Higgs $h$ we obtain the Higgs Yukawa couplings and the two-Higgs two-fermion couplings. The Yukawa 
coupling part of the Lagrangian reads

$$
-\mathcal{L}^{h f f}=y h\left(\begin{array}{c}
t_{L} \\
T_{L} \\
X_{L}^{2 / 3} \\
\tilde{T}_{L}
\end{array}\right) \underbrace{\left(\begin{array}{cccc}
0 & 0 & 0 & 0 \\
0 & s c & s c & \frac{1-2 s^{2}}{\sqrt{2}} \\
0 & s c & s c & \frac{1-2 s^{2}}{\sqrt{2}} \\
0 & \frac{1-2 s^{2}}{\sqrt{2}} & \frac{1-2 s^{2}}{\sqrt{2}} & -2 s c
\end{array}\right)}_{G_{h f \bar{f}}}\left(\begin{array}{c}
t_{R} \\
T_{R} \\
X_{R}^{2 / 3} \\
\tilde{T}_{R}
\end{array}\right)+\text { h.c. }
$$

For the two-Higgs two-fermion interactions we find

$$
-\mathcal{L}^{h h f f}=\frac{y}{2 f} h^{2}\left(\begin{array}{c}
t_{L} \\
T_{L} \\
X_{L}^{2 / 3} \\
\tilde{T}_{L}
\end{array}\right) \underbrace{\left(\begin{array}{cccc}
0 & 0 & 0 & 0 \\
0 & 1-2 s^{2} & 1-2 s^{2} & -2 \sqrt{2} s c \\
0 & 1-2 s^{2} & 1-2 s^{2} & -2 \sqrt{2} s c \\
0 & -2 \sqrt{2} s c & -2 \sqrt{2} s c & -2\left(1-2 s^{2}\right)
\end{array}\right)}_{G_{h h f \bar{f}}}\left(\begin{array}{c}
t_{R} \\
T_{R} \\
X_{R}^{2 / 3} \\
\tilde{T}_{R}
\end{array}\right)+\text { h.c. }
$$

After the rotation into the mass eigenstate basis, the two matrices $G_{h f \bar{f}}$ and $G_{h h f \bar{f}}$ yield the single and double Higgs couplings to fermions, respectively, which will be needed for the calculation of the single and double composite Higgs production cross sections through gluon fusion.

\subsection{Constraints from electroweak precision data and flavor physics}

The strongest experimental constraints on composite Higgs models still come from the electroweak precision measurements at the $Z$ pole mass at LEP. A convenient description of LEP precision data is given in terms of the parameters $\epsilon_{1}, \epsilon_{2}, \epsilon_{3}$ and $\epsilon_{b}$ [62-64]. These parameters are on the one hand measured with high precision [65], and on the other hand can easily be computed theoretically. In addition to the SM contribution present in the decoupling limit $f \rightarrow \infty$, the MCHM5 contributes to the $\epsilon$ parameters through three different effects. The first beyond the SM (BSM) effect arises from the modified coupling of the Higgs to $W$ and $Z$ gauge bosons, which induces a logarithmically divergent contribution to the oblique parameters $T$ and $S$, or equivalently to $\epsilon_{1}$ and $\epsilon_{3}$. The contribution is cut-off by the mass $m_{\rho}$ of the first composite vector resonance [66],

$$
\Delta \epsilon_{1}^{\mathrm{IR}}=-\frac{3 \alpha\left(M_{Z}\right)}{16 \pi \cos ^{2} \theta_{W}} \xi \log \left(\frac{m_{\rho}^{2}}{m_{h}^{2}}\right), \quad \Delta \epsilon_{3}^{\mathrm{IR}}=\frac{\alpha\left(M_{Z}\right)}{48 \pi \sin ^{2} \theta_{W}} \xi \log \left(\frac{m_{\rho}^{2}}{m_{h}^{2}}\right) .
$$

The second effect is the direct contribution of the vector $\rho$ and axial-vector $a$ resonances to the $S$ parameter, which in the MCHM is found to be (see ref. [67])

$$
\Delta \epsilon_{3}^{\mathrm{UV}}=\frac{m_{W}^{2}}{m_{\rho}^{2}}\left(1+\frac{m_{\rho}^{2}}{m_{a}^{2}}\right) \cong 1.36 \frac{m_{W}^{2}}{m_{\rho}^{2}} .
$$

In the second equality, we have used the relation $m_{a} / m_{\rho} \cong 5 / 3$, obtained in the fivedimensional realization of the model [40]. The third and last contribution to electroweak 

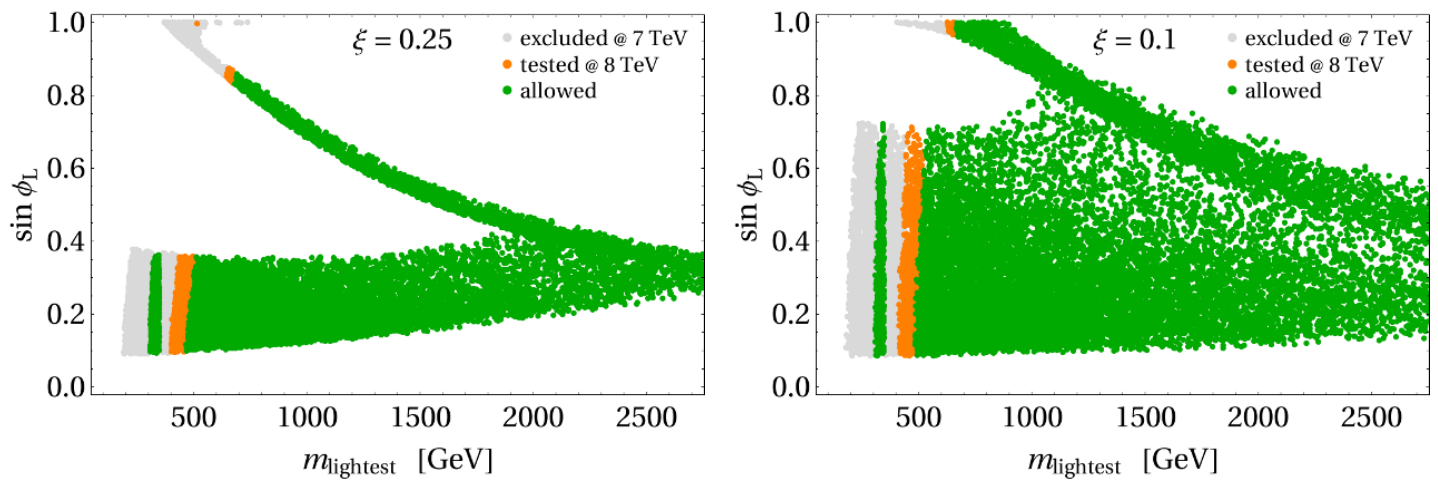

Figure 4. A sample of parameters passing the $\chi^{2}$-test of electroweak precision observables, displaying the compositeness of the left-handed top versus the mass of the lightest top partner, for $\xi=0.25$ (left) and $\xi=0.1$ (right). The points in light gray do not pass the direct collider constraints. Points in orange/medium gray pass the present constraints but will be tested by the LHC running at $8 \mathrm{TeV}$ with an integrated luminosity of $15 \mathrm{fb}^{-1}$, see section 4.2 .

precision parameters comes from the top partners at one loop, giving contributions both to the $T$ parameter and the $Z-\bar{b}-b$ vertex, i.e. respectively to $\epsilon_{1}$ and $\epsilon_{b}$ [43, 68-70]. Computing the precise value of these contributions requires the numerical diagonalisation of the mass matrix of the top quark and its partners, which depends on the parameters $\Delta_{L}, \Delta_{R}, M_{0}, y$ and $f$. The requirement that the top mass matches the measured value $m_{t}=173.3 \mathrm{GeV}$ allows, however, to express the corrections to $\epsilon_{1}$ and $\epsilon_{b}$ in terms of four dimensionless parameters,

$$
\Delta \epsilon_{1}^{\text {fermions }}=f_{1}\left(\xi, \phi_{L}, \phi_{R}, R\right), \quad \Delta \epsilon_{b}^{\text {fermions }}=f_{b}\left(\xi, \phi_{L}, \phi_{R}, R\right)
$$

where $\xi, \phi_{L}, \phi_{R}$ are defined above and $R=\left(M_{0}+y f\right) / M_{0}$. The function $f_{1}$ is computed exactly at one loop, while for $f_{b}$ only the longitudinal polarisations of the gauge bosons are taken into account in the loop. The values obtained in this way are consistent with the full one-loop result of ref. [70]. The agreement of the model with experimental data is then assessed through a $\chi^{2}$ test, described in detail in appendix C. The latest electroweak precision data are used, including the 2012 update of the $W$ mass. Fixing the Higgs mass to $m_{h}=125 \mathrm{GeV}$, the model is completely determined by the five parameters $\xi, \phi_{L}, \phi_{R}, R$ and $m_{\rho}$. Over the latter four a scan is performed for $\xi$ fixed to two representative values, namely 0.25 and 0.1 . The results are displayed in figure 4 for the left-handed compositeness angle $\phi_{L}$ versus the mass of the lightest top partner. Note that the value of $R$ is bounded by the requirement that $y<4 \pi$ and that we impose furthermore the constraint $\left|V_{t b}\right|>0.77$ [71]. In figure 5, the whole spectrum of composite fermions is shown for a sample of parameters passing the EWPT. The green points correspond to $B$, the red ones to $X^{5 / 3}$, and the blue points for each set of parameters denote the top partners $T, X^{2 / 3}$ and $\tilde{T}$, which cannot be properly distinguished one from another once the rotation in the physical basis is performed. At leading order in $v / f$, composite fermions within an electroweak doublet have, however, the same mass, so that the green points describe approximately the mass of 

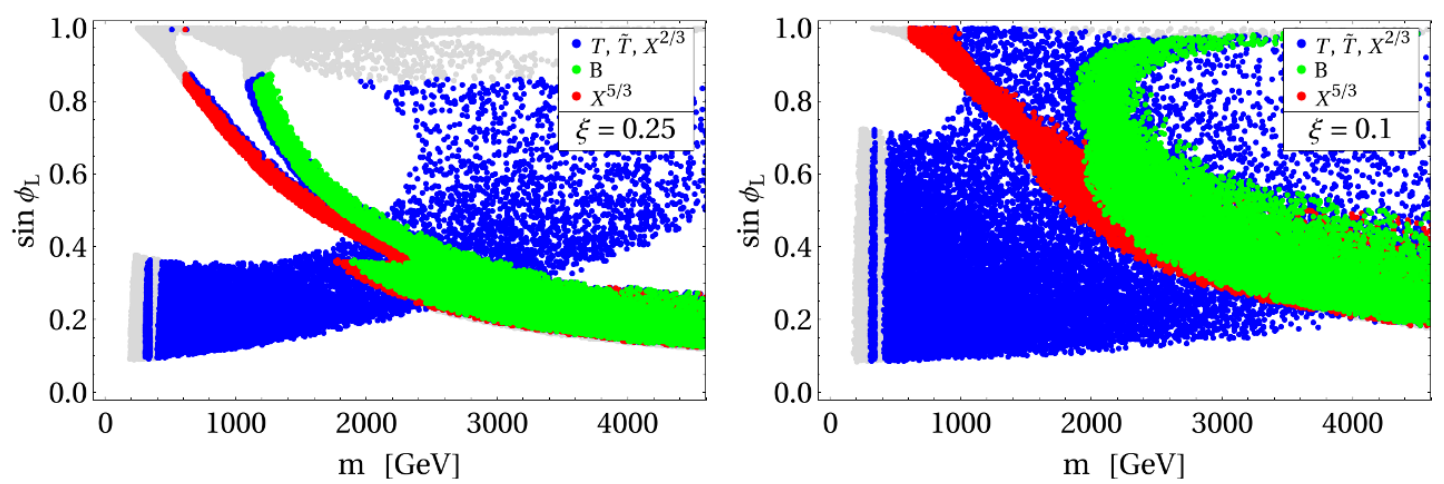

Figure 5. Physical mass spectrum of the composite fermions for a sample of points passing the electroweak precision tests, as a function of the left-handed top compositeness for $\xi=0.25$ (left) and $\xi=0.1$ (right). The blue/dark gray points are top-like fermions (charge $+2 / 3$ ), the green/fair gray points bottom-like (charge $-1 / 3$ ), and the red/medium gray ones correspond to the exotic $X$ $($ charge $+5 / 3)$. Light gray points are excluded by present collider constraints, see section 4.2.

the $(T, B)$ doublet and the red ones the mass of the $\left(X^{5 / 3}, X^{2 / 3}\right)$ doublet. The blue points far from the red and green regions in figure 5 can therefore be interpreted as singlets $\tilde{T}$.

There are two regions of the parameter space compatible with EWPT where in addition at least one of the top partners is light, as generically needed in order to have a light enough Higgs. The first region corresponds to low values of the top compositeness angle $\phi_{L}$, where the lightest top partner is typically the singlet $\tilde{T}$. In this case the fermion bidoublet is always heavier than $1.5 \mathrm{TeV}$ (see figure 5) and decouples. Note that the right-handed top must then be very composite in order to yield the correct top Yukawa coupling $y_{t}$. The second region corresponds to large values of $\sin \phi_{L}$, for which the top-bottom doublet becomes fully composite. In this second region, the 'custodian' doublet $X$ is very light, having a mass well below a TeV. Since the $X$ doublet contains an exotic charge $5 / 3$ fermion (which turns out to be the lightest new fermion for large $\sin \phi_{L}$ ), this region is very sensitive to direct collider constraints, as will be discussed in the next section. An intermediate region with moderate values of $\sin \phi_{L}$ is also allowed by precision data, although all new fermions are rather heavy, above $1 \mathrm{TeV}$, so it could be difficult to obtain a light enough Higgs in this region. A thorough discussion of the implications of top compositeness is contained in ref. [72] (see also ref. [73]). A comment is however in order: in ref. [72], when $t_{R}$ mixes with a $(\mathbf{1}, \mathbf{1})$ of $\mathrm{SU}(2)_{L} \times \mathrm{SU}(2)_{R}$ (which is the case in MCHM5) a highly composite righthanded top is not accompanied by anomalously light fermionic resonances, because there is no custodial partner of the state mixing with $t_{R}$, the latter being $\tilde{T}$ in MCHM5. Indeed one can write from eq. (4.16) $M_{\tilde{T}}=\Delta_{R} / \sin \phi_{R} \rightarrow \Delta_{R}$ when $\sin \phi_{R} \rightarrow 1$, implying that $\tilde{T}$ is not necessarily light in presence of a strongly composite $t_{R}$. However, EWPT select a light $\tilde{T}$ for large $\sin \phi_{R}$, as discussed for example in ref. [69].

Note finally that the constraints on the parameter space from electroweak precision data can be significantly relaxed by extending the fermion sector of the model [70].

Additional constraints on the model come from flavor physics. Composite Higgs models generically give rise to four-fermion operators which contribute to flavor-changing processes 
and to electric dipole moments. Low values of the compositeness scale $f$ as considered in this paper are allowed if the strong sector is flavor-symmetric, so that MFV can be implemented [74]. In this case both flavor-changing processes and electric dipole moments are inhibited, but the MFV assumption requires a large degree of compositeness also for light quarks, which are therefore sizably coupled to the strong sector resonances, leading to a different phenomenology. Experimental constraints can be described in an effective formalism, in which four-fermion operators arise after integrating out the vector resonances. The most relevant operator is

$$
\frac{g_{\rho}^{2}}{4 m_{\rho}^{2}}\left(\sin \phi_{L}\right)^{4}\left(\bar{q}_{L} \gamma^{\mu} t^{a} q_{L}\right)\left(\bar{q}_{L} \gamma_{\mu} t^{a} q_{L}\right)
$$

where $t^{a}$ are the generators of $\mathrm{SU}(3)_{c}$, which imposes a constraint on the size of the mixing angle $\phi_{L}$. From the most recent experimental dijet angular distributions [75], the bound is

$$
\frac{g_{\rho}^{2}}{4 m_{\rho}^{2}}\left(\sin \phi_{L}\right)^{4} \lesssim \frac{2 \pi}{(7.5 \mathrm{TeV})^{2}} \quad \Rightarrow \quad\left(\sin \phi_{L}\right)^{2} \lesssim \frac{f}{1.5 \mathrm{TeV}}
$$

or equivalently $\sin \phi_{L} \lesssim 0.6$ for $\xi=0.25$ and $\sin \phi_{L} \lesssim 0.7$ for $\xi=0.1$. Similar bounds apply to the compositeness of right-handed quarks. Minimal Flavor Violation with left-handed compositeness is in addition strongly constrained by EWPT [74].

However, it has been recently pointed out [76] that it is possible to treat the top differently from the light quarks, thus deviating from MFV. Flavor bounds are still satisfied, but since the first two generations are mostly elementary the constraints from EWPT and from searches for compositeness are relaxed. In this setup left-handed and right-handed top compositeness are both viable, and the phenomenology is expected to be analogous to the case where the strong sector is flavor-anarchic, given that the light generations are mostly elementary.

\subsection{Constraints from searches for heavy fermions}

Expanding the composite Yukawa coupling we obtain the leading interactions between one heavy fermion and two SM particles, which mediate the decay of the heavy states

$$
\begin{aligned}
\mathcal{L}_{\mathrm{Y}}= & y s_{L} c_{R}\left(\bar{b}_{L} \pi^{-}+\frac{\bar{t}_{L}}{\sqrt{2}}\left(h-i \pi^{0}\right)\right) \tilde{T}_{R}+y \frac{s_{R}}{\sqrt{2}} \bar{X}_{2 / 3 L}\left(h+i \pi^{0}\right) t_{R}+\text { h.c. } \\
& +\frac{y}{\sqrt{2}} s_{R} c_{L} \bar{T}_{L}\left(h-i \pi^{0}\right) t_{R}+y s_{R} c_{L} \bar{B}_{L} t_{R} \pi^{-}-y s_{R} \bar{X}_{5 / 3 L} t_{R} \pi^{+}+\text {h.c. },
\end{aligned}
$$

where we have already performed the rotations $\propto \phi_{L, R}$. From the Goldstone equivalence theorem then follow the leading order branching ratios (in the limit $M_{\psi} \gg m_{Z}, m_{h}$ )

$$
\begin{aligned}
\mathrm{BR}(\tilde{T} \rightarrow W b) & =\frac{1}{2}, & \operatorname{BR}(\tilde{T} \rightarrow Z t)=\mathrm{BR}(\tilde{T} \rightarrow h t) & =\frac{1}{4} ; \\
\operatorname{BR}\left(X^{2 / 3} \rightarrow Z t\right) & =\operatorname{BR}\left(X^{2 / 3} \rightarrow h t\right)=\frac{1}{2}, & \operatorname{BR}\left(X^{5 / 3} \rightarrow W t\right) & =1 ; \\
\operatorname{BR}(T \rightarrow Z t) & =\operatorname{BR}(T \rightarrow h t)=\frac{1}{2}, & \operatorname{BR}(B \rightarrow W t) & =1 .
\end{aligned}
$$



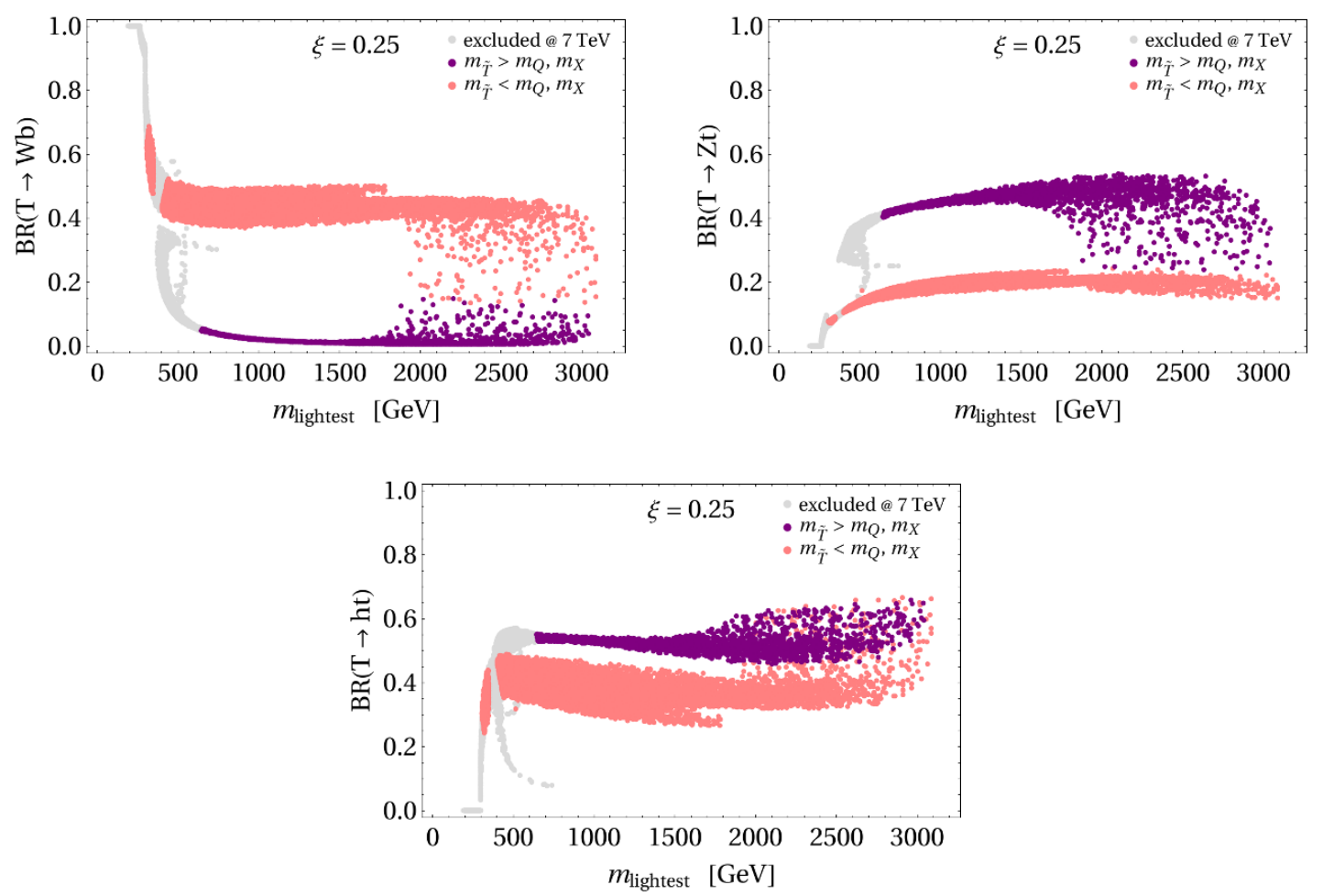

Figure 6. Branching ratios of the lightest top partner into $W^{+} b$ (upper left), $Z t$ (upper right) and $h t$ (lower) as a function of its mass for $\xi=0.25$. Points for which the singlet (doublet) is the lightest top partner are shown in pink/medium gray (purple/dark gray).

However, in our analysis of the electroweak and collider constraints we will keep all orders in $\xi$, by performing a full numerical diagonalization of the mass matrix in the top sector and computing the couplings of the mass eigenstates to gauge bosons and to the Higgs boson in the unitary gauge. Complete formulae for the partial decay widths of heavy fermions into SM fields are given in appendix D. As we will see, for the relatively large values of $\xi$ that we consider, sizeable corrections to the leading approximations listed above arise. In figure 6 we show the branching ratios of the lightest top partner as a function of its mass for $\xi=0.25$, for a set of points in parameter space which are compatible with EWPT. The pink points are the branching ratios in case the lightest top partner is the singlet. The purple points correspond to the lightest partner being the doublet. Compared to the approximate formulae the branching ratios into $h t$ are a bit enhanced while the ones into $Z t$ are somewhat reduced.

In certain regions of the parameter space, some fermionic resonances can be very light, thus rendering constraints from direct searches for heavy fermions at the LHC and Tevatron relevant. The experimental collaborations have performed several searches for pairproduced heavy fermions, with subsequent decay into the final states $W b W b, Z t Z t, W t W t$. Since pair-production of the heavy fermions is a QCD process, the cross section $\sigma(p p, p \bar{p} \rightarrow$ $\psi \bar{\psi})$, with $\psi$ being a generic heavy fermion, only depends on $M_{\psi}$. The constraint from e.g. a search for $\psi \bar{\psi} \rightarrow W b W b$ at the LHC will read

$$
\sigma_{Q C D}(p p \rightarrow \psi \bar{\psi}) \times \mathrm{BR}(\psi \rightarrow W b)^{2} \leq \sigma_{\exp },
$$




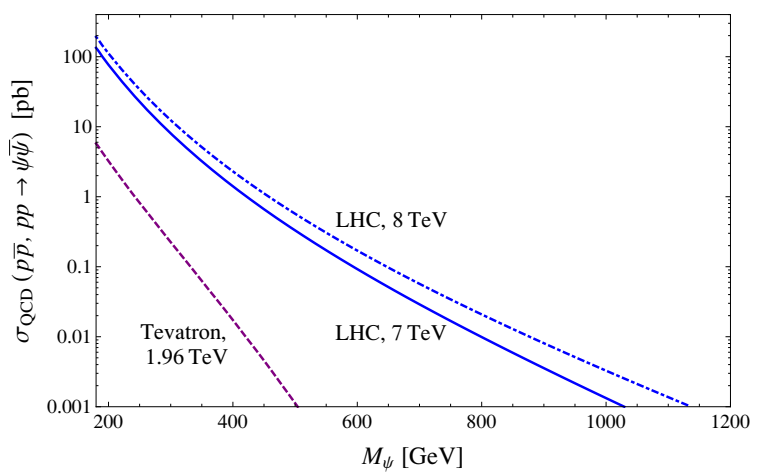

Figure 7. Cross sections for QCD pair production of heavy fermions at approximate NNLO, at the Tevatron (dashed), at LHC7 (solid) and at LHC8 (dot-dashed). The cross sections were computed using HATHOR [77], and MSTW2008 PDFs.

where $\sigma_{\exp }$ is the upper bound on the cross section, as given by the experiment for each value of the resonance mass. The QCD pair-production cross sections were obtained at approximate next-to-next-to-leading order (NNLO) [77], and are shown in figure 7. We remark that also single production of heavy fermions can give complementary, relevant constraints (see e.g. the fourth among refs. [14-22] for a detailed analysis), however no such search has been published by ATLAS and CMS yet. Therefore, we do not discuss single production.

Note that the branching ratios are non-trivial only for the top partners $T, X^{2 / 3}$ and $\tilde{T}$, whereas $B$ and $X^{5 / 3}$ decay with unity branching ratio into $t W^{\mp}$. We summarize in table 3 all the searches for pair-produced heavy fermions that we included in our analysis. The analyses of $t W t W$ final states, although intended by the experiments to be searches for heavy charge $-1 / 3$ quarks such as the $B$, apply straightforwardly also to the $X^{5 / 3}$, which decays into the same final state. ${ }^{8}$

The region of the parameter space corresponding to $\sin \phi_{L} \sim 1$ is the most constrained by direct searches. The lightness of the $X^{5 / 3}$ fermion in this case (see figure 5 ) is prohibited by both Tevatron and LHC searches in $W t W t$ final states. For a lower degree of compositeness of the left-handed top quark, the lightest top partner is the singlet $\tilde{T}$, which decays in all three final states $W b, Z t$ and $h t$. The Tevatron only has enough sensitivity to exclude top partners below $300 \mathrm{GeV}$, while the most stringent LHC constraints (i.e. those based on the full 2011 luminosity) start at $350 \mathrm{GeV}$. This leaves a region of the parameter space in the range $m_{\tilde{T}} \in[300,350] \mathrm{GeV}$ which is not directly excluded by present constraints, see figure 4 .

In addition to the present exclusion limits, we show in figure 4 an estimate of the reach of the LHC in 2012. The increase in energy enhances significantly the production cross

\footnotetext{
${ }^{8}$ Note that the decay products of $B \bar{B}$ and $X^{5 / 3} \bar{X}^{5 / 3}$ would have different spatial configurations. For example, same-sign leptons necessarily stem either from $X^{5 / 3}$ or from its antiparticle, while in the case of $B \bar{B}$ production each of the same-sign leptons arises from a different heavy particle. However, since in the current searches only basic cuts on single objects are applied, this kind of kinematic differences is expected to give negligible effects on the exclusion limits.
} 


\begin{tabular}{|c|c|c|c|c|}
\hline exp. & search & $L\left[\mathrm{fb}^{-1}\right]$ & range in $M_{\psi}[\mathrm{GeV}]$ & ref. \\
\hline \multirow[t]{5}{*}{ CMS [78-82] } & $W b W b$ (1 lepton) & 4.7 & {$[400,625]$} & CMS-PAS-EXO-11-099 \\
\hline & $W b W b$ (2 leptons) & 5.0 & {$[350,600]$} & arXiv: 1203.5410 \\
\hline & $W t W t$ & 1.14 & {$[350,550]$} & CMS-PAS-EXO-11-036 \\
\hline & $W t W t$ & 4.9 & {$[450,650]$} & arXiv: 1204.1088 \\
\hline & $Z t Z t$ & 1.14 & {$[250,550]$} & arXiv: 1109.4985 \\
\hline \multirow[t]{4}{*}{ ATLAS [83-86] } & $W b W b$ & 1.04 & {$[250,500]$} & arXiv: 1202.3076 \\
\hline & $W q W q$ & 1.04 & {$[300,500]$} & arXiv: 1202.3389 \\
\hline & $W t W t$ (1 lepton) & 1.04 & {$[300,600]$} & arXiv: 1202.6540 \\
\hline & $W t W t$ (2 leptons) & 1.04 & {$[300,600]$} & arXiv: 1202.5520 \\
\hline \multirow[t]{2}{*}{$\mathrm{CDF}[87,88]$} & $W b W b$ & 5.6 & {$[180,500]$} & arXiv:1107.3875 \\
\hline & $W t W t$ & 4.8 & {$[260,425]$} & arXiv:1101.5728 \\
\hline
\end{tabular}

Table 3. List of experimental searches for pair-produced heavy fermions that we included in our analysis of collider constraints.

section of heavy fermion pairs (see figure 7). On the other hand, the present exclusion limits quoted by ATLAS and CMS will be modified due to the changes in the background and to the additional integrated luminosity. Backgrounds in searches for top partners are dominated by top quark pair production, which is increased by $42 \%$ when going from 7 to $8 \mathrm{TeV}$ c.m. energy at the LHC. The search strategy relies on a cut on the $t \bar{t}$ invariant mass, whose distribution is not significantly affected by the increase in energy, as explicitly checked using MadGraph 5 [89]. The upper limit on the top partner production cross section is therefore softened in the Gaussian approximation by a factor $\sqrt{1.42} \cong 1.19$. The total luminosity of $15 \mathrm{fb}^{-1}$ expected to be attained in 2012 is nevertheless tightening the limit on the cross section, lowering it by a square root factor of the luminosity in every channel. More refined searches after the LHC upgrade to $14 \mathrm{TeV}$ will be needed in order to explore the full parameter space [14-22].

\section{Single Higgs production in MCHM5}

The cross section for single Higgs production in MCHM5 can be readily derived by noting that we can directly apply eq. (3.2) since the Higgs kinetic term is canonically normalized. We therefore only need the determinant of the mass matrix of top-like fermions in eq. (4.14), which reads

$$
\operatorname{det} \mathcal{M}^{\dagger}(H) \mathcal{M}(H)=\frac{M_{0}^{4} y^{2} f^{2} \sin ^{2} \phi_{L} \sin ^{2} \phi_{R}}{8 \cos ^{2} \phi_{L} \cos ^{2} \phi_{R}}\left(M_{0}+y f\right)^{2} \sin ^{2}\left(\frac{2 H}{f}\right),
$$

and has the form of eq. (3.13). Thus we obtain $A_{1}=(2 / v)(1-2 \xi) / \sqrt{1-\xi}$ (where we have used $\left.\sin ^{2}(\langle H\rangle / f)=\xi\right)$, and

$$
\frac{\sigma(p p \rightarrow h)}{\sigma(p p \rightarrow h)_{\mathrm{SM}}}=\left(\frac{1-2 \xi}{\sqrt{1-\xi}}\right)^{2},
$$


which is valid to all orders in $\xi$. Equation (5.2) is independent of the details of the fermion spectrum. While this holds exactly only in the low-energy theorem limit, as discussed in section 3.3 we expect that retaining the full mass dependence will give corrections to the cross section at most of a few percent. This is confirmed by a full computation of the cross section in which the dependence on the fermion masses is retained, as shown in figure 8. The figure shows the cross section of single Higgs production through gluon fusion including new fermionic resonances, normalized to the SM cross section computed with finite $m_{t}$, as a function of the mass of the lightest resonance. ${ }^{9}$ Note that the QCD $K$-factors ${ }^{10}$ cancel out under the assumption that the higher order corrections are the same in both cases $^{11}$ (see ref. [53]). A parameter scan has been performed, selecting only points that satisfy EWPT. Among these, points that satisfy all current collider bounds are shown in green, whereas gray points are already excluded. In orange we show points currently allowed, but that will be excluded by searches for heavy fermions at the end of the LHC8 run if no excess is observed. The agreement with the prediction of the low-energy theorem in eq. (5.2), shown as a black line, confirms that the cross section is to an excellent approximation independent of the details of the spectrum, and is fixed only by $\xi$. The sensitivity to the composite couplings is at most $2 \% \times \sigma_{\mathrm{SM}}$ for light top partners, in agreement with our previous estimate, and vanishes for heavy partners. We conclude that for single Higgs production the LET provides a very accurate cross section for any spectrum of the extra fermions.

Finally we remark that the result in eq. (5.2) coincides with the one obtained considering only the Higgs nonlinearities, i.e. rescaling the SM cross section by $c^{2}$, where $c$ is the correction to the top Yukawa in the limit where fermionic resonances are heavy and thus their effects negligible (see table 1). This is a consequence of the cancellation discussed above.

\subsection{Effect of non-minimal operators}

We can add to the minimal partial compositeness Lagrangian in eq. (4.10) the following operators

$$
\Delta \mathcal{L}=i y_{L}^{\prime}\left(\bar{\psi}_{L} \Sigma^{T}\right) \not D\left(\Sigma \psi_{L}\right)+i y_{R}^{\prime}\left(\bar{\psi}_{R} \Sigma^{T}\right) \not D\left(\Sigma \psi_{R}\right)
$$

where the covariant derivative reads $D_{\mu}=\partial_{\mu}-i g^{\prime} X B_{\mu}(X=2 / 3)$. The most convenient way to discuss these operators is to perform the following field redefinition,

$$
\psi_{L, R} \rightarrow \zeta(x)^{T} \psi_{L, R}
$$

\footnotetext{
${ }^{9}$ We have compared our results in the SM limit to the ones obtained with HIGLU [90].

${ }^{10}$ The $K$-factor is defined as the ratio of the higher-order cross section to the leading order cross section.

${ }^{11}$ This assumption is valid only at next-to-leading order (NLO) QCD. At NNLO QCD different mass scales play a role. Furthermore, the correct matching of the strong coupling constant in the effective and the full theory has to be performed. In ref. [53], however, it was shown that for parameters similar to ours the differences in the $K$-factors are of the order of a few percent only, so that the SM NNLO $K$-factor can safely be applied also to the MCHM5 case.
} 


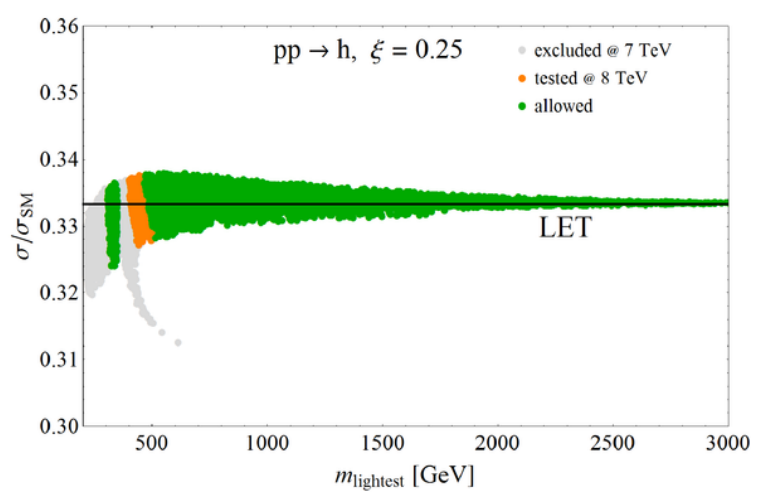

Figure 8. The MCHM5 cross section for single Higgs production through gluon fusion (including the exact dependence on top and heavy fermion masses), normalised to the SM cross section (computed retaining the $m_{t}$ dependence), as a function of the mass of the lightest fermion resonance $m_{\text {lightest }}$ for $m_{h}=125 \mathrm{GeV}$. The compositeness parameter has been chosen $\xi=0.25$. Green/dark gray points are allowed, gray points are excluded by current collider constraints, whereas orange/fair gray points will be tested by LHC8 in 2012. For comparison, the cross section ratio computed with the LET, eq. (5.2), is shown as a black line.

where $\zeta(x)$ was defined in eq. (4.6). Upon this transformation, the Lagrangian reads (omitting kinetic terms of elementary fields, and gauge interactions)

$$
\begin{aligned}
\mathcal{L}_{f}+\Delta \mathcal{L} \rightarrow & i \bar{\psi}_{L} \not \partial \psi_{L}+i \bar{\psi}_{L} \gamma^{\mu} \zeta(x)\left(\partial_{\mu} \zeta^{T}(x)\right) \psi_{L}+(L \rightarrow R) \\
& -y f\left(\bar{\psi}_{L} \Sigma_{0}^{T}\right)\left(\Sigma_{0} \psi_{R}\right)-M_{0} \bar{\psi}_{L} \psi_{R}+\text { h.c. } \\
& +i y_{L}^{\prime}\left(\bar{\psi}_{L} \Sigma_{0}^{T}\right) \not D\left(\Sigma_{0} \psi_{L}\right)+(L \rightarrow R) \\
& -\Delta_{L} \overline{\mathcal{Q}}_{L} \zeta^{T}(x) \psi_{R}-\Delta_{R} \bar{\psi}_{L} \zeta(x) \mathcal{T}_{R}+\text { h.c. }
\end{aligned}
$$

Thus we need to rescale the singlet $\tilde{T}$ to make it canonically normalized, $\tilde{T}_{L, R} \rightarrow$ $\tilde{T}_{L, R} / \sqrt{1+y_{L, R}^{\prime}}$. Let us now focus on how the amplitude for $g g \rightarrow h$ is modified by the new operators. It is easy to verify that the Higgs derivative interactions contained in eq. (5.5) do not contribute to the amplitude for single Higgs production, because they are antisymmetric in the fermion fields [29]. Therefore we can simply apply the low-energy theorem. From the fermion mass matrix, which reads

$$
\mathcal{M}=\left(\begin{array}{cccc}
0 & \Delta_{L} \frac{1+\cos (H / f)}{2} & \Delta_{L} \frac{\cos (H / f)-1}{2} & \Delta_{L} \frac{\sin (H / f)}{\sqrt{2}} \frac{1}{\sqrt{1+y_{R}^{\prime}}} \\
-\frac{\sin (H / f)}{\sqrt{2}} \Delta_{R} & M_{0} & 0 & 0 \\
-\frac{\sin (H / f)}{\sqrt{2}} \Delta_{R} & 0 & M_{0} & 0 \\
\frac{\cos (H / f)}{\sqrt{1+y_{L}^{\prime}}} \Delta_{R} & 0 & 0 & \frac{M_{0}+y f}{\sqrt{1+y_{L}^{\prime}} \sqrt{1+y_{R}^{\prime}}}
\end{array}\right)
$$

we obtain

$$
\operatorname{det} \mathcal{M}^{2}(H)=\frac{\Delta_{L}^{2} \Delta_{R}^{2} f^{2} M_{0}^{2} y^{2}}{8\left(1+y_{L}^{\prime}\right)\left(1+y_{R}^{\prime}\right)} \sin ^{2}\left(\frac{2 H}{f}\right),
$$

which implies that the amplitude for $g g \rightarrow h$ is not sensitive to the value of $y_{L, R}^{\prime}$, see eq. (3.13). On the other hand, the Higgs derivative interactions in eq. (5.5) contribute in 
general to the pair production process, because they enter box diagrams. Therefore the cross section for $g g \rightarrow h h$ will be sensitive to $y_{L, R}^{\prime}$. In the following section, however, we consider the minimal Lagrangian, setting $y_{L, R}^{\prime}=0$.

Finally we comment about the contribution of the exotic state $X^{5 / 3}$ to the amplitudes for $g g \rightarrow h, h h$. In the field basis of eq. (5.5) the Higgs appears only in elementary/composite mixing terms and in derivative interactions (thus showing manifestly its pseudo-GB nature). Since $X^{5 / 3}$ does not mix with any elementary field, there is no contribution to the amplitudes from the mixing terms. On the other hand, it is easy to check explicitly that Higgs derivative interactions do not involve $X^{5 / 3}$. We conclude that the exotic state does not contribute at all to the amplitudes for single and double Higgs production via gluon fusion.

\section{Double Higgs production in MCHM5}

In this section we discuss the cross section for $p p \rightarrow h h$ first in the LET approximation, and subsequently retaining the full dependence on the masses of the fermions running in the loops.

\subsection{LET cross section}

From the determinant of the fermion mass matrix in eq. (5.1) we can compute $A_{2}=$ $\left(-2 / v^{2}\right) /(1-\xi)$, which determines the hhgg coupling via fermion loops. This, together with the form of $A_{1}$ previously derived and with the expression of the $h^{3}$ coupling given in table 1, allows us to write down the amplitude for $g g \rightarrow h h$ at all orders in $\xi$ :

$$
C_{\mathrm{MCHM} 5}^{\mathrm{LET}}(\hat{s})=\frac{3 m_{h}^{2}}{\hat{s}-m_{h}^{2}}\left(\frac{1-2 \xi}{\sqrt{1-\xi}}\right)^{2}-\frac{1}{1-\xi} .
$$

Thus analogously to single Higgs production, the LET cross section for Higgs pair production is insensitive to the details of the heavy fermion spectrum, and is fixed only by $\xi$. The corresponding $p p \rightarrow h h$ cross section at LHC14, normalized to the SM cross section (also computed in the infinite $m_{t}$ limit) was shown as a function of $\xi$ in the left panel of figure 3 .

\subsection{Enhancement of the cross section}

We have seen that for small values of $\xi$ single Higgs production in the MCHM5 is suppressed compared to the SM while double Higgs production is enhanced. The behavior of single Higgs production becomes clear from the LET result given in eq. (5.2). In double Higgs production the Higgs pair is either produced through Higgs bosons coupling to the gluons through triangle loops or through boxes. In the former case, in the SM we only have a diagram with a Higgs subsequently decaying into two Higgs bosons, while in composite Higgs models there is an additional triangle diagram due to the two-Higgs two-fermion coupling. In the amplitude for Higgs pair production the parts coming from the triangle containing the triple Higgs coupling and from the box diagrams interfere destructively. In the MCHM5 amplitude where these two contributions are modified by $((1-2 \xi) / \sqrt{1-\xi})^{2}$, the additional diagram with the two-Higgs two-fermion coupling proportional to $\xi$ can 


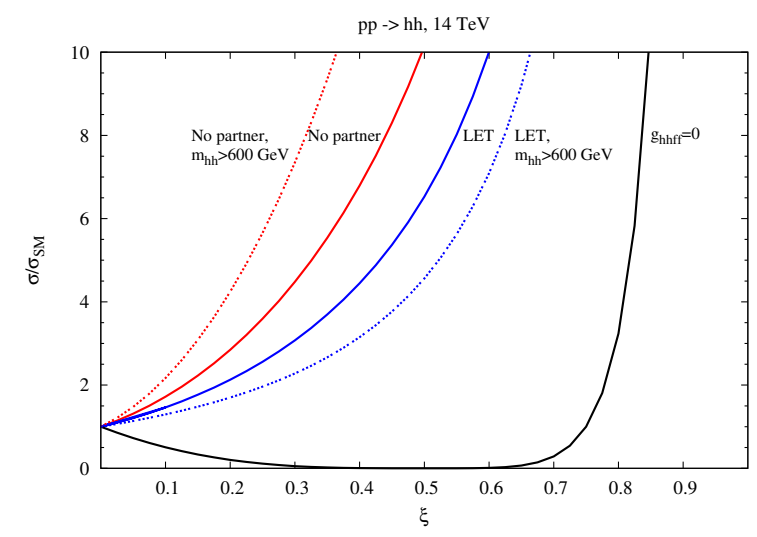

Figure 9. The cross section for double Higgs production in MCHM5 normalized to the SM as a function of $\xi$ for three different approximations. Red: in the limit of heavy top partners keeping the full top quark mass dependence. Blue: LET. Black: setting the two-Higgs two-fermion coupling to zero. The red/blue dotted lines show the same as the red/blue full lines after application of an invariant mass cut of $m_{h h} \geq 600 \mathrm{GeV}$.

hence have order one effects so that it governs the total cross section. This can be inferred from figure 9 which shows the double Higgs production MCHM5 cross section normalized to the SM as a function of $\xi$ for three different approximations. The red line has been obtained in the limit of heavy top partners keeping the full top quark mass dependence, the blue line is the LET result, and the black line, finally, is obtained by explicitly setting the two-Higgs two-fermion coupling to zero. In this case the cross section ratio is given by $((1-2 \xi) / \sqrt{1-\xi})^{4}$ both for the LET and for the approximation where the top quark mass dependence has been kept. The dotted lines in the figure have been obtained by applying an invariant mass cut of $m_{h h} \geq 600 \mathrm{GeV}$. After application of the cut the discrepancy in the cross section results for the two approximations becomes even worse, see also the discussion in section 6.4.

\subsection{Full 1-loop cross section}

In the triangle diagrams which contribute to double Higgs production the gluons couple to the total spin $S_{z}=0$ along the $z$-axis, whereas the box diagrams involve $S_{z}=0$ and $S_{z}=2$ couplings. The amplitude for the process can hence be expressed in terms of independent form factors $F_{\triangle}, F_{\square}, F_{\square, 5}$ associated with spin 0 and $G_{\square}, G_{\square, 5}$ associated with spin 2 . The total partonic cross section is given by

$$
\begin{aligned}
\hat{\sigma}_{g g \rightarrow h h}= & \frac{\alpha_{s}^{2}}{1024(2 \pi)^{3}} \frac{1}{\hat{s}^{2}} \int_{\hat{t}_{-}}^{\hat{t}_{+}} d \hat{t}\left[\left|\sum_{i=1}^{4} \sum_{j=1}^{4}\left(g_{h \bar{q}_{i} q_{j}}^{2} G_{\square}\left(m_{i}, m_{j}\right)+g_{h \bar{q}_{i} q_{j}, 5}^{2} G_{\square, 5}\left(m_{i}, m_{j}\right)\right)\right|^{2}\right. \\
& \left.+\left|\sum_{i=1}^{4}\left(C_{i, \triangle} F_{\triangle}\left(m_{i}\right)+\sum_{j=1}^{4}\left(g_{h \bar{h}_{i} q_{j}}^{2} F_{\square}\left(m_{i}, m_{j}\right)+g_{h \bar{q}_{i} q_{j}, 5}^{2} F_{\square, 5}\left(m_{i}, m_{j}\right)\right)\right)\right|^{2}\right],
\end{aligned}
$$

with the integration limits

$$
\hat{t}_{ \pm}=-\frac{\hat{s}}{2}\left(1-2 \frac{m_{h}^{2}}{\hat{s}} \mp \sqrt{1-\frac{4 m_{h}^{2}}{\hat{s}}}\right),
$$



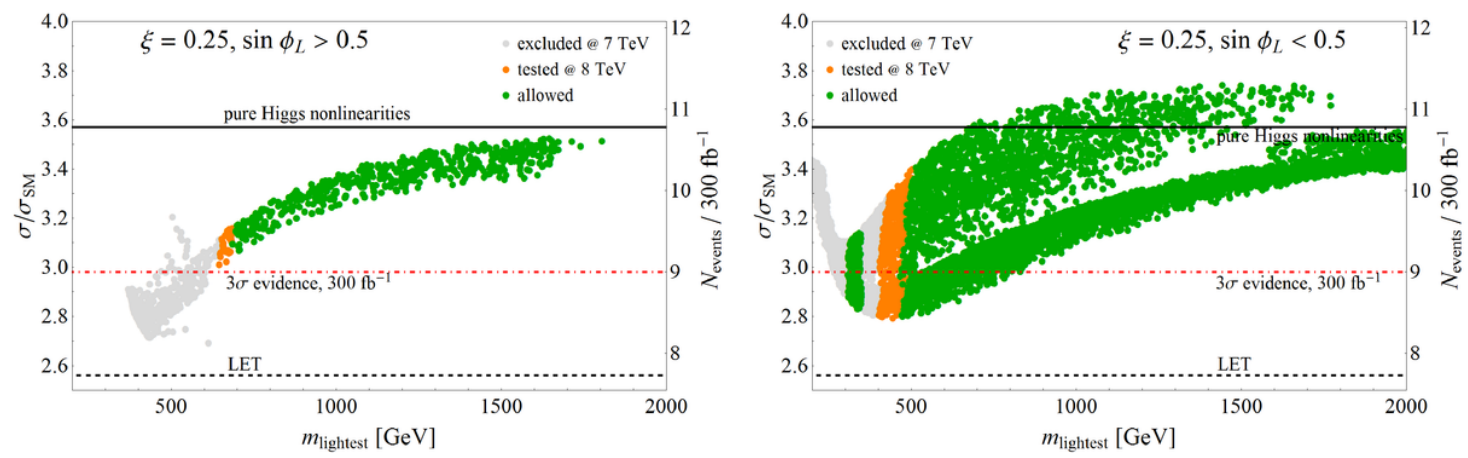

Figure 10. The cross section for double Higgs production through gluon fusion normalized to the $\mathrm{SM}$ as function of the mass of the lightest resonance of the heavy top sector, for $m_{h}=125 \mathrm{GeV}$. The compositeness parameter has been chosen $\xi=0.25$. Green/dark gray (gray) dots denote points which pass (do not pass) all current constraints, whereas orange/fair gray dots correspond to points that will be tested by LHC8. The left panel shows points for which $X^{2 / 3}$ is the lightest top partner (as a consequence of $t_{L}$ being largely composite), whereas for points in the right panel the lightest top partner is typically the singlet $\tilde{T}$. The black solid (dashed) line corresponds to the result in the limit of heavy top partners keeping the full top mass dependence (to the LET result as in figure 3). The expected number of events in the $h h \rightarrow b \bar{b} \gamma \gamma$ final state after all cuts at LHC14 with $L=300 \mathrm{fb}^{-1}$ is also shown, along with the $3 \sigma$ evidence threshold (dot-dashed line), see text for details.

where $\hat{s}$ denotes the partonic c.m. energy. The triangle and box form factors are given in appendix E. The various couplings appearing in eq. (6.2) are also defined there. We have explicitely verified that in the SM limit our result agrees with ref. [33]. The hadronic cross section is obtained by convolution with the parton distribution function of the gluon in the proton, see eq. (3.23).

\subsection{Numerical analysis}

For the numerical analyis we have performed, after fixing $\xi$ to one of the benchmark values $\xi=0.25$ or $\xi=0.1$, a scan in the parameter set $\left(\phi_{L}, \phi_{R}, R\right)$ and retained only the points which fulfill the constraints from EWPT. By this we mean that there exists a value of $m_{\rho} \in[1.5 \mathrm{TeV}, 4 \pi f]$ such that the configuration $\left(\xi, \phi_{L}, \phi_{R}, R, m_{\rho}\right)$ passes EWPT at $99 \%$ CL. For this set of points we show in figure 10 for $m_{h}=125 \mathrm{GeV}$ and $\xi=0.25$ the double Higgs production cross section normalized to the SM as a function of the lightest top partner mass. The dependence on the masses of the loop particles has been fully taken into account. The black solid line shows the result in the limit of heavy partners, keeping only the top contribution (with full mass dependence) in the loop, while the black dashed line corresponds to the LET result in figure 3. The green (gray) dots are points which pass (do not pass) the current constraints from Tevatron and LHC data, whereas orange points will be tested by LHC8.

Some comments are in order. First of all, we find a sizeable dependence of the cross section on the spectrum of the heavy fermions with $2.7 \lesssim \sigma / \sigma_{\mathrm{SM}} \lesssim 3.7$. We recall that both the LET cross section and the cross section in the limit of heavy partners only depend on $\xi$. 

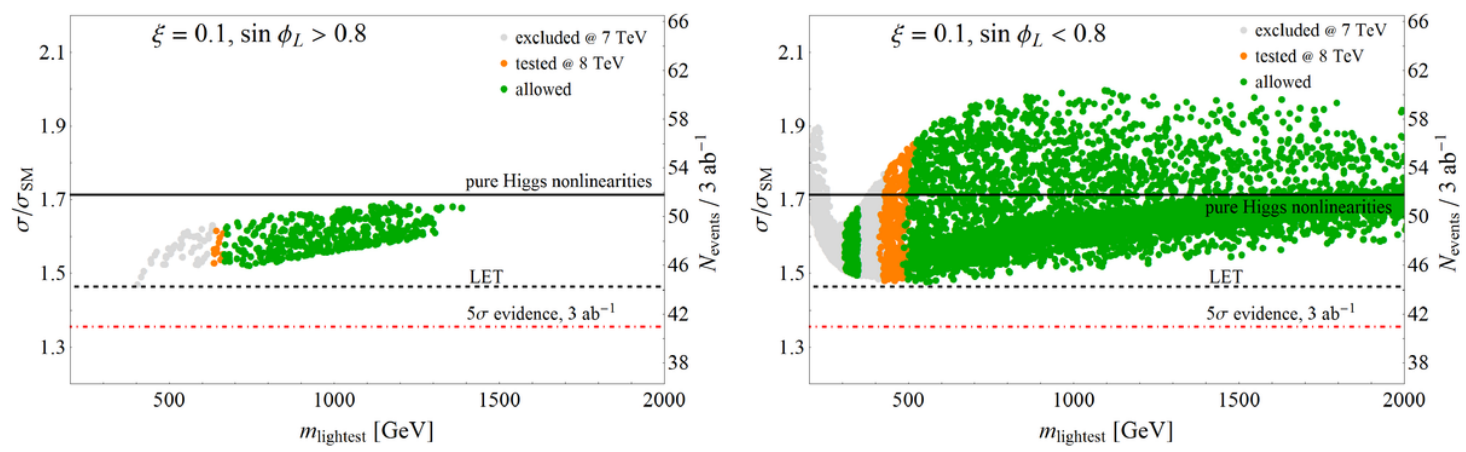

Figure 11. Cross section for double Higgs production through gluon fusion normalised to the $\mathrm{SM}$ as function of the mass of the lightest resonance of the heavy top sector, for $\xi=0.1$ and $m_{h}=125 \mathrm{GeV}$. Points are split in the two panels depending on the degree of compositeness of $t_{L}$. The meaning of the dots and lines is the same as in figure 10, except that we assumed an integrated luminosity $L=3 \mathrm{ab}^{-1}$ at LHC14. The dot-dashed line corresponds to the $5 \sigma$ discovery threshold, see text for details.

The LET approximation, however, severely underestimates the ratio $\sigma / \sigma_{\mathrm{SM}}$, and this effect is even worse if we refer directly to the cross section, since we are consistently normalizing the LET cross section for MCHM5 to $\sigma_{\mathrm{SM}}\left(m_{t} \rightarrow \infty\right)$, which is $\sim 20 \%$ smaller than the full result. On the other hand, the result in the limit of heavy partners, while keeping the full top mass dependence [36], overestimates the cross section in the region $m_{\text {lightest }} \lesssim 1 \mathrm{TeV}$, which is compatible with a Higgs as light as $125 \mathrm{GeV}$. For large values of the partner masses, of course, the cross section tends to the value obtained including only top loops (with top couplings following the 'trigonometric' rescalings given in table 1).

It should be noted that we have not taken into account higher-order QCD corrections. They have been calculated at NLO for SM and MSSM Higgs pair production in ref. [91] in the heavy top mass limit. However, they cannot be taken over here as we have the additional diagram with the two-Higgs two-fermion coupling and more seriously box diagrams with different loop particle masses. For heavy loop particle masses we do not expect the corrections to be too different from the SM case, so that they approximately cancel out in the ratio of the two cross sections.

In figure 11 we show the corresponding results for a lower value of $\xi=0.1$, which corresponds to $f \simeq 800 \mathrm{GeV}$. Due to the larger value of $f$, the cross section is less enhanced compared to the SM. Similarly to the case $\xi=0.25$ the LET underestimates the cross section, although in a less dramatic way than in the previous case.

To estimate the reach of the $14 \mathrm{TeV}$ run of the LHC on double Higgs production, we focus on the final state $h h \rightarrow b \bar{b} \gamma \gamma$, which was shown to be the most promising for a light Higgs boson [34, 92-94]. Reference [94] found that assuming a luminosity $L=$ $600 \mathrm{fb}^{-1}, 6$ signal events could be obtained after all cuts, with a background of 11 events. We estimate the expected number of signal events for MCHM5 by computing $\sigma(p p \rightarrow$ $h h) \times \mathrm{BR}(h h \rightarrow b \bar{b} \gamma \gamma)$ for each point in the parameter space (taking into account the QCD 
production $K$-factor ${ }^{12}$ of 1.9 and the non-standard Higgs branching ratios) and multiplying it times the acceptance for all cuts as computed in ref. [94] for the SM. This rough approximation cannot of course replace a full analysis of the effects of cuts in the MCHM5 case, which however goes beyond the scope of this work. We therefore apply the simplified procedure for an illustratory purpose. We also quote the number of events needed for a $3(5) \sigma$ evidence with $L=300(3000) \mathrm{fb}^{-1}$, based on the background estimate of ref. [94] with the requirement of one $b$-tagged jet. Notice that this is likely to be conservative, because the analysis of reducible backgrounds (whose sum is larger than the irreducible $b \bar{b} \gamma \gamma)$ performed in ref. [94] made use of efficiencies and misidentification probabilities, in particular for $b$-jets, that have since then been improved by ATLAS and CMS. We find that a $3 \sigma$ excess can be obtained already with $300 \mathrm{fb}^{-1}$ if $\xi=0.25$, except perhaps in some regions of the parameter space with a very light top partner. A $5 \sigma$ discovery would be possible at the LHC luminosity upgrade for a more moderate value $\xi=0.1$.

We note that in the recent ref. [37] two $b$-tagged jets were required, and the efficiency and rejection probabilities for $b$-tagging were updated to current values. However, since we are only interested in a rough estimate of the LHC reach, we conservatively adopt the numbers of ref. [94]. Furthermore, a realistic analysis of the instrumental backgrounds relevant to $b \bar{b} \gamma \gamma$ would require a detailed knowledge of the detector properties, which is out of the reach of a theoretical analysis. See also ref. [95] for a study of the $b \bar{b} \tau \tau$ final state, and ref. [96] for an analysis of the $b \bar{b} W W \rightarrow b \bar{b} \ell \nu j j$ channel.

Additionally, we studied if applying a cut on the invariant mass $m_{h h}$ could be useful to measure deviations from the SM cross section. Therefore, in figure 12 we show the same as figure 10 but after an invariant mass cut of $m_{h h} \geq 600 \mathrm{GeV}$ has been applied. As can be inferred from the plot the composite cross section is more enhanced compared to the SM than without application of a cut, see also figure 10. On the other hand the absolute value of the cross section after cuts becomes very small. The plots reveal, however, another interesting feature. While for masses of the lightest top partner above $2 \mathrm{TeV}$ the total cross section is reasonably well approximated by the cross section where only Higgs nonlinearities are considered, see figure 10, this is not the case any more after application of cuts. This can be inferred from figure 12 by comparing the full result, given by the points, to the black line, which is the ratio of the double Higgs production cross section considering only Higgs nonlinearities to the SM cross section (the full top dependence has been included in both cases). So we conclude that not only the heavy top partner limit in the total cross section of double Higgs production is a rather bad approximation unless the top partners are really heavy, but this approximation becomes even worse when a cut on $m_{h h}$ is applied. The latter, however, may be relevant in the experimental analyses to enhance the signal to background ratio and to extract information on the couplings involved in the process.

\footnotetext{
${ }^{12}$ As stated above the SM QCD corrections to double Higgs production cannot be translated trivially to the composite Higgs case. Assuming the top partners to be heavy we expect, however, the error not to be too large by applying the SM K-factor to MCHM5 double Higgs production. Concerning the diagram involving the two-Higgs two-fermion coupling we explicitly verified that it hardly changes the QCD corrections compared to the SM ones.
} 

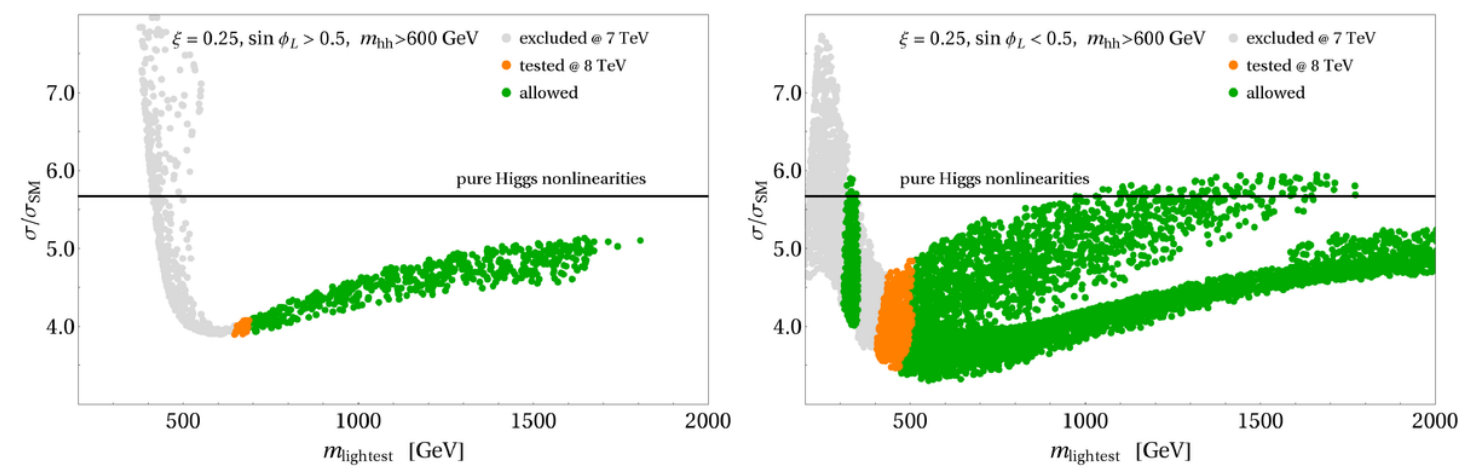

Figure 12. The cross section for double Higgs production through gluon fusion after an invariant mass cut $m_{h h} \geq 600 \mathrm{GeV}$, normalized to the SM for $m_{h}=125 \mathrm{GeV}$, as function of the mass of the lightest resonance of the heavy top sector. The compositeness parameter has been chosen $\xi=0.25$. Green/dark gray (gray) dots denote points which pass (do not pass) all current constraints, whereas orange/fair gray dots correspond to points that will be tested by LHC8. The left panel shows points for which $X^{2 / 3}$ is the lightest top partner, whereas for the points in the right panel the lightest top partner is typically the singlet $\tilde{T}$. The black solid line corresponds to the result obtained considering only Higgs nonlinearities, i.e. in the limit of heavy top partners and keeping the full top mass dependence.

\section{Conclusions}

Models of electroweak symmetry breaking aiming at giving a rationale for the stability of the weak scale under radiative corrections predict an extended top sector at an energy scale typically below a TeV. Carrying color and electric charges, these top partners are naively expected to give significant corrections to the loop-induced couplings of the Higgs boson to massless photons and gluons. We examined this question in the context of composite Higgs models where the Higgs boson emerges as a Goldstone boson from a strongly-interacting theory. We first extended the well-known SM Higgs low-energy theorem that gives a simple way to estimate the contribution of heavy particles to the Higgs couplings to photons and gluons and we then checked the accuracy of this LET approximation to an explicit full oneloop computation taking into account the contributions of all fermionic resonances. We confirmed that in composite models there is actually a quite efficient cancellation for the contribution of the top partners to the single Higgs production cross section, which deviates by no more than a few percents from the result obtained taking into account the Higgs nonlinearities only. For single production, the LET provides a very accurate prediction of the cross section. The situation is, however, totally different for double Higgs production for which the LET approximation is not reliable any more, and deviates from the true result by up to $50 \%$. The top partners also significantly reduce the enhancement of the $g g \rightarrow h h$ cross section over the SM that was previously computed taking into account the strong dynamics effects only. This dependence on the top partner spectrum and couplings gives an indirect access to this sector that will complement the information gathered from direct searches as well as from electroweak and flavor precision data. 
The recent discovery of the Higgs boson puts the identification of its true nature on the immediate agenda of high-energy physics and a careful study of the top sector can bring invaluable information. If the Higgs is a composite object, then $t \bar{t} h$ and $g g \rightarrow h h$ will be important channels that can give access to the top partners and where large deviations compared to the SM predictions are expected.

\section{Acknowledgments}

We thank A. Azatov, R. Contino, C. Englert, M. Farina, G. Panico, D. Pappadopulo, R. Rattazzi, M. Rauch, J. Santiago, M. Spira and A. Wulzer for fruitful discussions, and J. Serra for comments about the manuscript. M. G. is supported by the Schweizer Nationalfonds and by the European Commission under the contract PITN-GA-2010-264564 LHCPhenoNet. R. G. and M. M. are supported by the DFG SFB/TR9 Computational Particle Physics. R. G. acknowledges financial support from the Landesgraduiertenkolleg. The work of C. G. and E. S. has been partly supported by the European Commission under the ERC Advanced Grant 226371 Mass TeV and the contract PITN-GA-2009-237920 UNILHC. E. S. has been supported in part by the European Commission under the ERC Advanced Grant 267985 DaMeSyFla.

\section{A Derivation of the $h g g, h h g g$ and $h \gamma \gamma$ couplings in the SILH formalism}

In this section we derive the expressions of the couplings $h g g$, hhgg and $h \gamma \gamma$ in the SILH formalism.

\section{A.1 The hgg and hhgg couplings}

Our starting point is eq. (3.2). We also stress that since we are working in a general basis where $c_{r} \neq 0$, the relation between $\langle H\rangle$ and $v$ is non-trivial, as can be read off the $W$ boson mass term,

$$
m_{W}^{2}=\frac{g^{2} v^{2}}{4}, \quad \text { with } \quad v^{2}=\langle H\rangle^{2}\left(1+\frac{c_{r}}{4} \frac{\langle H\rangle^{2}}{f^{2}}\right) .
$$

We assume for definiteness the presence of one or more vector-like top partners, which upon integration contribute to $c_{g}$, and identify the light mass eigenstate of the heavy fermion mass matrix $\mathcal{M}$ with the top quark, whose mass reads

$$
m_{t}(H)=\frac{y_{t} H}{\sqrt{2}}\left(1-c_{y}^{(t)} \frac{H^{2}}{2 f^{2}}\right)
$$

where $c_{y}^{(t)}$ parameterizes the correction to the SM top Yukawa coupling. The coefficients $A_{1,2}$ in eq. (3.2) can be related to $c_{y}^{(t)}$ and $c_{g}$ by separating the contribution (to the $h g g$ and $h$ hgg coupling, respectively) of the top quark, which involves $c_{y}^{(t)}$, from that of top 
partners, which is parameterized by $c_{g}$. The results are

$$
\begin{aligned}
\frac{1}{2}\langle H\rangle A_{1} & =\frac{1}{2}\left(\frac{\partial}{\partial \log H} \log \operatorname{det} \mathcal{M}^{2}(H)\right)_{H=v}=1-c_{y}^{(t)} \frac{v^{2}}{f^{2}}+3 c_{g} \frac{y_{t}^{2}}{m_{\rho}^{2}} v^{2}, \\
\frac{1}{2}\langle H\rangle^{2} A_{2} & =\frac{1}{2}\left(\left(\frac{\partial^{2}}{\partial(\log H)^{2}}-\frac{\partial}{\partial \log H}\right) \log \operatorname{det} \mathcal{M}^{2}(H)\right)_{H=v}=-1-c_{y}^{(t)} \frac{v^{2}}{f^{2}}+3 c_{g} \frac{y_{t}^{2}}{m_{\rho}^{2}} v^{2},
\end{aligned}
$$

where we work at $\mathcal{O}\left(1 / f^{2}\right) \cdot{ }^{13}$ Note that the 'implicit' expressions containing the determinant are in practice more useful than the explicit ones written in terms of $c_{y}^{(t)}$ and $c_{g}$, because using the former avoids diagonalizing the heavy fermion mass matrix, a rather complicated task in presence of multiple top partners.

In eq. (3.2) we have assumed that $h$ has a canonical kinetic term. However, in the SILH Lagrangian the operators proportional to $c_{H}$ and $c_{r}$ correct the Higgs kinetic term as follows

$$
\Delta \mathcal{L}_{h k i n}=\frac{1}{2 f^{2}}\left(c_{H}+\frac{c_{r}}{4}\right)(\langle H\rangle+h)^{2} \partial_{\mu} h \partial^{\mu} h,
$$

which also contains Higgs derivative interactions. At first order in $\xi$, these can be eliminated by the nonlinear redefinition [26]

$$
h \rightarrow h-\frac{\xi}{2}\left(c_{H}+\frac{c_{r}}{4}\right)\left(h+\frac{h^{2}}{v}+\frac{h^{3}}{3 v^{2}}\right),
$$

which leaves $h$ canonically normalized. Notice that in a nonlinear $\sigma$-model, the Higgs is canonically normalized at all orders, which corresponds to the relation $c_{H}=-c_{r} / 4$. Performing the transformation in eq. (A.6), we arrive at the effective coupling of the Higgs to one and two gluons, eqs. (3.5) and (3.6), respectively. The invariance of these expressions under the reparameterization in eq. (2.2) can be easily verified by using eqs. (A.3) and (A.4), respectively.

\section{A.2 The $h \gamma \gamma$ coupling}

Starting from eq. (3.9), recalling the expression of the $W$ boson mass in eq. (A.1) and taking into account the rescaling needed to make the Higgs kinetic term canonical, see eq. (A.6), it is straightforward to obtain eq. (3.10). Similarly to eq. (A.3), $A_{1}$ can be related to $c_{y}^{(t)}$ and $c_{\gamma}$ (assuming that all contributions to $c_{\gamma}$ come from the fermion sector)

$$
\frac{1}{2}\langle H\rangle A_{1}=\frac{1}{2}\left(\frac{\partial}{\partial \log H} \log \operatorname{det} \mathcal{M}^{2}(H)\right)_{H=v}=1-c_{y}^{(t)} \frac{v^{2}}{f^{2}}+c_{\gamma} \frac{g^{2}}{m_{\rho}^{2}} v^{2} \frac{1}{2 Q_{t}^{2}} .
$$

Plugging eq. (A.7) into eq. (3.10), the invariance under the reparameterization in eq. (2.2) becomes explicit.

\footnotetext{
${ }^{13}$ In the second and third term of each of eqs. (A.3) and (A.4) we have used the fact that the distinction between $\langle H\rangle$ and $v$ expressed by eq. (A.1) is higher order in $\xi$ there.
} 


\section{B The SILH coefficients for the Littlest Higgs and MCHM4}

In this section we give the minimal details which are needed to compute the coefficients of the SILH Lagrangian relevant to Higgs production via gluon fusion in the Littlest Higgs and in the minimal composite Higgs model with fermions in the spinorial representation.

\section{B.1 Littlest Higgs}

The Littlest Higgs model [97] is based on the coset $\mathrm{SU}(5) / \mathrm{SO}(5)$. We consider here a variation where only one $\mathrm{U}(1)$ group is gauged, as this eliminates one source of custodial breaking and thus relaxes the tension with EWPT suffered by the original model. In ref. [98] it was shown that a scale as low as $f \sim 1.2 \mathrm{TeV}$ is allowed in this case. This, however, leaves an extra singlet Goldstone boson in the spectrum, whose effects will be ignored in the following. ${ }^{14}$ The $\Sigma$ field reads

$$
\Sigma(x)=e^{2 i \Pi / f} \Sigma_{0}, \quad \Pi=\left(\begin{array}{ccc}
\eta /(2 \sqrt{5}) & H / \sqrt{2} & \varphi \\
H^{\dagger} / \sqrt{2} & -2 \eta / \sqrt{5} & H^{T} / \sqrt{2} \\
\varphi^{\dagger} & H^{*} / \sqrt{2} & \eta /(2 \sqrt{5})
\end{array}\right), \quad \Sigma_{0}=\left(\begin{array}{c}
\mathbb{1}_{2} \\
1 \\
\mathbb{1}_{2}
\end{array}\right),
$$

where $H$ is the Higgs doublet, $\varphi$ is a complex triplet and $\eta$ is a singlet. An $\mathrm{SU}(2)_{1} \times$ $\mathrm{SU}(2)_{2} \times \mathrm{U}(1)_{Y}$ subgroup of the global symmetry is gauged and is spontaneously broken at the scale $f$ to the diagonal $\mathrm{SU}(2)_{L} \times \mathrm{U}(1)_{Y}$. In eq. (B.1) we omitted the GBs that get eaten by the heavy $\mathrm{SU}(2)$ triplet of vectors. The two-derivative Lagrangian reads

$\mathcal{L}=\frac{f^{2}}{8} \operatorname{Tr}\left[\left(D_{\mu} \Sigma\right)^{\dagger} D^{\mu} \Sigma\right], \quad D_{\mu} \Sigma=\partial_{\mu} \Sigma-i \sum_{j=1,2} g_{j} W_{j}^{a}\left(Q_{j}^{a} \Sigma+\Sigma Q_{j}^{a T}\right)-i g^{\prime} B_{\mu}(Y \Sigma+\Sigma Y)$,

with the gauged generators given by

$$
Q_{1}^{a}=\left(\begin{array}{c}
\sigma^{a} / 2 \\
\end{array}\right), \quad Q_{2}^{a}=\left(\begin{array}{c} 
\\
-\sigma^{a *} / 2
\end{array}\right), \quad Y=\operatorname{diag}(1 / 2,1 / 2,0,-1 / 2,-1 / 2) .
$$

The SM fermions are taken to transform only under $\mathrm{SU}(2)_{1} \times \mathrm{U}(1)_{Y}$.

The coefficients $c_{H}$ and $c_{r}$ receive contributions of three different kinds. The first arises from the nonlinear $\sigma$ model structure. Using

$$
m_{W}^{2}=\frac{1}{2} g^{2} f^{2} \sin ^{2}\left(\frac{\langle H\rangle}{\sqrt{2} f}\right), \quad g=\frac{g_{1} g_{2}}{\sqrt{g_{1}^{2}+g_{2}^{2}}},
$$

we find $c_{H}^{\sigma}=1 / 6$ and $c_{r}^{\sigma}=-4 c_{H}^{\sigma}=-2 / 3$.

The second contribution comes from integrating out the heavy vector triplet. The procedure has been described in detail in ref. [47], and we simply apply it to the case under study, obtaining $c_{H}^{v}=1 / 4$ and $c_{r}^{v}=-1$, in agreement with ref. [28].

\footnotetext{
${ }^{14}$ Additional sources of symmetry breaking are needed in order to give the singlet a potential.
} 
The third and last contribution arises from integrating out heavy scalars. Since we also need to compute $c_{6}$, we write down the scalar potential up to order $H^{6}$. Neglecting $g^{\prime}$, the relevant terms are

$$
\begin{aligned}
V= & c_{+}\left\{f^{2}\left|\varphi_{i j}+\frac{i}{4 f}\left(H_{i} H_{j}+H_{j} H_{i}\right)\right|^{2}\right. \\
& \left.\quad-\frac{1}{6 f^{2}}|H|^{6}+\frac{i}{2 f}|H|^{2}\left(\varphi_{i j} H_{i}^{*} H_{j}^{*}-\varphi_{i j}^{*} H_{i} H_{j}\right)-\frac{4}{3}\left|\varphi_{i j}\right|^{2}|H|^{2}\right\} \\
& +c_{-}\left\{f^{2}\left|\varphi_{i j}-\frac{i}{4 f}\left(H_{i} H_{j}+H_{j} H_{i}\right)\right|^{2}\right. \\
& \left.\quad-\frac{1}{6 f^{2}}|H|^{6}-\frac{i}{2 f}|H|^{2}\left(\varphi_{i j} H_{i}^{*} H_{j}^{*}-\varphi_{i j}^{*} H_{i} H_{j}\right)-\frac{4}{3}\left|\varphi_{i j}\right|^{2}|H|^{2}\right\},
\end{aligned}
$$

where the coefficient $c_{+}$receives contributions from $g_{1}$, whereas $c_{-}$from $g_{2}$ and from the top Yukawa sector. In general, starting from a Lagrangian of the form

$$
\begin{aligned}
\mathcal{L}_{\Phi}= & -\Phi^{a *} \square \Phi^{a}-\left(M^{2}-\beta_{2}|H|^{2}\right) \Phi^{a *} \Phi^{a}+\left(\beta f \Phi^{a *} H^{T} \epsilon \frac{\sigma^{a}}{2} H+\text { h.c. }\right) \\
& +\left(\frac{\beta_{4}}{f} \Phi^{a *} H^{T} \epsilon \frac{\sigma^{a}}{2} H|H|^{2}+\text { h.c. }\right)
\end{aligned}
$$

$\left(\epsilon=i \sigma^{2}\right)$ and integrating out $\Phi$ one obtains $c_{H}^{s}=\beta^{2} f^{4} /\left(2 M^{4}\right)$ and $c_{r}^{s}=2 \beta^{2} f^{4} / M^{4}$. In addition, there is a contribution to $c_{6}$,

$$
c_{6}^{\Phi}=-\frac{1}{\lambda}\left(\frac{\beta \beta_{4} f^{2}}{M^{2}}+\frac{\beta^{2} \beta_{2} f^{4}}{2 M^{4}}\right),
$$

where $\lambda$ is the Higgs quartic coupling (after the triplet has been integrated out). In the Littlest Higgs case we make the identifications

$$
M^{2}=\left(c_{+}+c_{-}\right) f^{2}, \quad \beta=\frac{1}{\sqrt{2}}\left(c_{-}-c_{+}\right), \quad \beta_{2}=\frac{4}{3}\left(c_{+}+c_{-}\right), \quad \beta_{4}=\frac{1}{\sqrt{2}}\left(c_{+}-c_{-}\right) .
$$

Therefore we find

$$
c_{H}^{s}=\frac{1}{4}\left(\frac{c_{-}-c_{+}}{c_{-}+c_{+}}\right)^{2}, \quad c_{r}^{s}=\left(\frac{c_{-}-c_{+}}{c_{-}+c_{+}}\right)^{2} .
$$

On the other hand,

$$
c_{6}=-\frac{1}{\lambda}\left(\frac{c_{+}+c_{-}}{6}\right)+c_{6}^{\Phi}=-\frac{2}{3}
$$

where we have used the expression of the quartic coupling $\lambda=c_{+} c_{-} /\left(c_{+}+c_{-}\right)$. Notice that in general the neutral component of $\varphi$ gets a nonzero VEV, which is strongly constrained by EWPT. Small values of $f \sim 1 \mathrm{TeV}$ in fact require the approximate condition $c_{+} \simeq c_{-}$ 
to be satisfied, which makes the triplet VEV very small ${ }^{15}$ [98]. We assume this condition to be realized, and therefore neglect effects due to the triplet VEV in our discussion.

Concerning the top sector, in addition to the doublet $q_{L}=\left(t_{L}, b_{L}\right)^{T}$ and to the singlet $t_{R}$ a pair of $\mathrm{SU}(2)$-singlet fermions $\tilde{T}_{L}, \tilde{T}_{R}$ with electric charge $Q=Y=2 / 3$ is introduced. The Yukawa Lagrangian then reads

$$
-\mathcal{L}_{Y}=\frac{\lambda_{1}}{2} f \bar{t}_{R} \epsilon_{i j k} \epsilon_{a b} \chi_{i} \Sigma_{j a} \Sigma_{k b}+\lambda_{2} f \overline{\tilde{T}}_{R} \tilde{T}_{L}+\text { h.c. }
$$

$(i, j, k=1,2,3$ and $a, b=4,5)$. Here $\chi$ is an $\mathrm{SU}(3)$ triplet, $\chi=\left(b_{L}, t_{L}, \tilde{T}_{L}\right)^{T}$. The fermion mass matrix in the Higgs background

$$
H=\left(\begin{array}{c}
0 \\
H / \sqrt{2}
\end{array}\right)
$$

reads

$$
-\mathcal{L}_{m}=\left(\bar{t}_{R} \overline{\tilde{T}}_{R}\right) \mathcal{M}\left(\begin{array}{c}
t_{L} \\
\tilde{T}_{L}
\end{array}\right)+\text { h.c. }, \quad \mathcal{M}=\left(\begin{array}{cc}
-\frac{i}{\sqrt{2}} \lambda_{1} f \sin \left(\frac{\sqrt{2} H}{f}\right) & \lambda_{1} f \cos ^{2}\left(\frac{H}{\sqrt{2} f}\right) \\
0 & \lambda_{2} f
\end{array}\right),
$$

implying

$$
\operatorname{det} \mathcal{M}^{\dagger} \mathcal{M}=\frac{1}{2} \lambda_{1}^{2} \lambda_{2}^{2} f^{4} \sin ^{2} \frac{\sqrt{2} H}{f} .
$$

This allows us to write the amplitude for $g g \rightarrow h h$ in the Littlest Higgs, in the low-energy theorem limit as

$$
C_{\mathrm{LET}}^{\mathrm{LH}}(\hat{s})=\frac{3 m_{h}^{2}}{\hat{s}-m_{h}^{2}}\left[1-\frac{3}{4} \xi\left(\frac{7}{3}+\left(\frac{c_{-}-c_{+}}{c_{-}+c_{+}}\right)^{2}\right)\right]-1-\frac{\xi}{4}\left(1+\left(\frac{c_{-}-c_{+}}{c_{-}+c_{+}}\right)^{2}\right) .
$$

We also note that in this case it is easy to diagonalize explicitly the fermion mass matrix at $\mathcal{O}\left(1 / f^{2}\right)$, obtaining

$$
-\mathcal{L}_{m}=m_{t}(H) \bar{t}_{R} t_{L}+m_{T}(H) \bar{T}_{R} T_{L}+\text { h.c. }
$$

where

$$
\begin{aligned}
& m_{t}(H)=\frac{y_{t} H}{\sqrt{2}}\left[1+\frac{H^{2}}{f^{2}}\left(-\frac{1}{3}+\frac{y_{t}^{2}}{4 \lambda_{T}^{2}}\right)\right], \\
& m_{T}(H)=f \lambda_{T}\left(1-\frac{H^{2}}{f^{2}} \frac{y_{t}^{2}}{4 \lambda_{T}^{2}}\right),
\end{aligned}
$$

with $y_{t}=\sqrt{2} \lambda_{1} \lambda_{2} / \sqrt{\lambda_{1}^{2}+\lambda_{2}^{2}}$ and $\lambda_{T}=\sqrt{\lambda_{1}^{2}+\lambda_{2}^{2}}$. In the notation of eq. (3.14), we have

$$
c_{y}^{(t)}=\frac{2}{3}-\frac{y_{t}^{2}}{2 \lambda_{T}^{2}}, \quad a_{T}=-\frac{y_{t}^{2}}{4 \lambda_{T}^{2}} .
$$

Thus $c_{y}^{(t)}-2 a_{T}=2 / 3=$ const., as must be the case since the factorization in eq. (3.13) holds.

\footnotetext{
${ }^{15}$ When $c_{+}=c_{-}$the potential does not contain any tadpole for $\varphi$.
} 


\section{B.2 MCHM4}

Similarly to what we did for MCHM5, we can apply directly eq. (3.2) to derive the $h g g$ and $h$ hgg effective couplings at all orders in $\xi$. For more details about the model we refer the reader to ref. [27], the notation of which we adopt here. The composite fermions are embedded into $\mathrm{SO}(5)$ spinors $\boldsymbol{4}_{1 / 6}$ as

$$
\psi_{L}=\left(\begin{array}{c}
q_{L 1} \\
-i T_{L} \\
-i B_{L}
\end{array}\right), \quad \psi_{R}=\left(\begin{array}{c}
Q_{R} \\
i t_{R 1} \\
i b_{R 1}
\end{array}\right)
$$

where $q_{L 1}=\left(t_{L 1}, b_{L 1}\right)^{T}$ and $Q_{R}=\left(T_{R}, B_{R}\right)^{T}$ are $\mathrm{SU}(2)_{L}$ doublets, and $T_{L}, B_{L}, t_{R 1}, b_{R 1}$ are singlets. In addition, an elementary doublet $q_{L 2}=\left(t_{L 2}, b_{L 2}\right)^{T}$ and singlet $t_{R 2}$ are present. The fermion Lagrangian reads

$$
-\mathcal{L}_{f}=y \bar{\psi}_{L} \Gamma^{M} \Phi_{M} \psi_{R}+f \lambda_{q} \bar{q}_{L, 2} Q_{R}+f \lambda_{t} \bar{T}_{L} t_{R 2}+\text { h.c. },
$$

where $\Phi=f(0,0,0, \sin (H / f), \cos (H / f))$, and $\Gamma^{M}$ are the Gamma matrices of $\mathrm{SO}(5)$. The mass matrix reads

$$
-\mathcal{L}_{m}=\left(\begin{array}{lll}
\bar{t}_{L 1} & \bar{t}_{L 2} & \bar{T}_{L}
\end{array}\right) \mathcal{M}\left(\begin{array}{c}
t_{R 1} \\
t_{R 2} \\
T_{R}
\end{array}\right)+\text { h.c., } \quad \mathcal{M}=f\left(\begin{array}{ccc}
y \sin (H / f) & 0 & y \cos (H / f) \\
0 & 0 & \lambda_{q} \\
y \cos (H / f) & \lambda_{t} & -y \sin (H / f)
\end{array}\right)
$$

from which we find

$$
\operatorname{det} \mathcal{M}^{\dagger} \mathcal{M}=\lambda_{q}^{2} \lambda_{t}^{2} y^{2} f^{6} \sin ^{2}(H / f) \quad \text { and } \quad A_{1}=\frac{2}{v} \sqrt{1-\xi}, \quad A_{2}=-\frac{2}{v^{2}} .
$$

Finally recalling the expression of the Higgs trilinear coupling in MCHM4, $\mathcal{L}_{h^{3}}=$ $-\left(m_{h}^{2} / 2 v\right) h^{3} \sqrt{1-\xi}$, we find the amplitude for Higgs pair production via gluon fusion in the low-energy theorem approximation

$$
C_{\mathrm{LET}}^{\mathrm{MCHM} 4}(\hat{s})=\frac{3 m_{h}^{2}}{\hat{s}-m_{h}^{2}}(1-\xi)-1 .
$$

\section{The $\chi^{2}$ test for electroweak precision observables}

We discuss here in detail the $\chi^{2}$ test used to constrain the parameters of the MCHM5 described in section 4.1. The best experimental determination of $\epsilon_{1}, \epsilon_{3}$ and $\epsilon_{b}$ still comes from the precision measurements at the $Z$ pole mass at LEP [65]:

$$
\begin{aligned}
& \epsilon_{1}^{(\exp )}=(5.4 \pm 1.0) \cdot 10^{-3}, \\
& \epsilon_{2}^{(\exp )}=(-8.9 \pm 1.2) \cdot 10^{-3}, \\
& \epsilon_{3}^{(\exp )}=(5.34 \pm 0.94) \cdot 10^{-3}, \\
& \epsilon_{b}^{(\exp )}=(-5.0 \pm 1.6) \cdot 10^{-3},
\end{aligned} \quad \rho=\left(\begin{array}{cccc}
1 & 0.60 & 0.86 & 0.00 \\
0.60 & 1 & 0.40 & -0.01 \\
0.86 & 0.40 & 1 & 0.02 \\
0.00 & -0.01 & 0.02 & 1
\end{array}\right)
$$


Here $\rho$ is the correlation matrix between the $\epsilon_{i}$ obtained from the appendix $\mathrm{E}$ of ref. [65], marginalising over the three parameters $m_{Z}, \alpha_{S}\left(m_{Z}\right)$ and $\Delta \alpha_{\text {had }}^{(5)}\left(m_{Z}\right) .{ }^{16}$ The status of electroweak precision observables did not change since then, except for the mass of the $W$ boson. The latter was recently updated based on Tevatron results [99, 100], and the new world average is now [101]

$$
m_{W}=80.385 \pm 0.015 \mathrm{GeV} .
$$

The parameter $\epsilon_{2}$ is the only one depending on the mass of the $W$ boson, through the term

$$
\epsilon_{2}=\frac{s_{0}^{2}}{1-2 s_{0}^{2}} \Delta r_{w}+\left[\text { terms independent of } m_{W}\right]
$$

where $s_{0}^{2}=0.23098$, and the measurement of $\Delta r_{w}$ is related to $m_{W}$ through

$$
\frac{\pi \alpha(0)}{\sqrt{2} m_{Z}^{2} G_{F}}\left(1-\Delta \alpha-\Delta r_{w}\right)^{-1}=\frac{m_{W}^{2}}{m_{Z}^{2}}\left(1-\frac{m_{W}^{2}}{m_{Z}^{2}}\right) .
$$

Here $\alpha(0)$ is the fine-structure constant and $G_{F}$ the Fermi constant, both known to high accuracy. Furthermore, $\Delta \alpha$ accounts for the running of the electroweak coupling between the low energy limit and the $Z$-pole mass. The uncertainty associated to it is important, but the shift in $\Delta r_{w}$ induced by the new value of $m_{W}$ is independent of $\Delta \alpha$. The change of $m_{W}$ and consequently $\epsilon_{2}$ between the LEP data of 2006 and the present value is then

\begin{tabular}{|c|c|c|}
\hline & 2006 & 2012 \\
\hline$m_{W}$ & $80.425 \pm 0.034 \mathrm{GeV}$ & $80.385 \pm 0.015 \mathrm{GeV}$ \\
\hline$\epsilon_{2}$ & $(-8.9 \pm 1.2) \cdot 10^{-3}$ & $(-7.9 \pm 0.9) \cdot 10^{-3}$ \\
\hline
\end{tabular}

The experimental values for the $\epsilon_{i}$ used in this paper are therefore

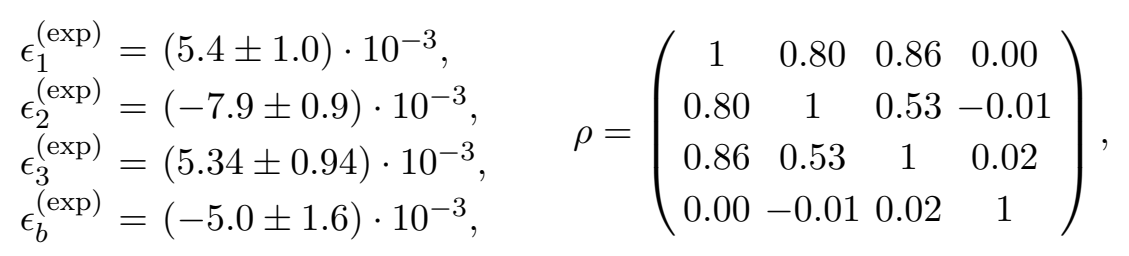

where we took into account the fact that $\epsilon_{1,3, b}$ and their covariances with $\epsilon_{2}$ are not affected by the new measurement of the $W$ mass.

On the theoretical side, the $\epsilon_{i}$ are predicted to take the values [43]

$$
\begin{aligned}
\epsilon_{1}^{(\mathrm{th})} & =\left[+5.66-0.86 \log \left(m_{h} / m_{Z}\right)\right] \cdot 10^{-3}+\Delta \epsilon_{1}^{\mathrm{IR}}+\Delta \epsilon_{1}^{\text {fermions }} \\
\epsilon_{2}^{(\mathrm{th})} & =\left[-7.11+0.16 \log \left(m_{h} / m_{Z}\right)\right] \cdot 10^{-3} \\
\epsilon_{3}^{(\mathrm{th})} & =\left[+5.25+0.54 \log \left(m_{h} / m_{Z}\right)\right] \cdot 10^{-3}+\Delta \epsilon_{3}^{\mathrm{IR}}+\Delta \epsilon_{3}^{\mathrm{UV}} \\
\epsilon_{b}^{(\mathrm{th})} & =-6.48 \cdot 10^{-3}+\Delta \epsilon_{b}^{\text {fermions }}
\end{aligned}
$$

\footnotetext{
${ }^{16}$ Alternatively, we could set the three extra parameters to their experimental best values. This would give slightly more stringent constraints.
} 
where the first numbers are the Standard Model corrections and the remaining contributions are given in section 4.1 for the MCHM5. For the computation, we used a top mass $m_{t}=173.3 \mathrm{GeV}$ and a Higgs mass of $m_{h}=125 \mathrm{GeV}$. The $\chi^{2}$ test is then defined as

$$
\chi^{2}\left(\xi, \phi_{L}, \phi_{R}, R, m_{\rho}\right)=\sum_{i, j}\left(\epsilon_{i}^{(\mathrm{th})}-\epsilon_{i}^{(\mathrm{exp})}\right) C_{i j}^{-1}\left(\epsilon_{j}^{(\mathrm{th})}-\epsilon_{j}^{(\mathrm{exp})}\right)
$$

where $C^{-1}$ is the inverse of the covariance matrix

$$
C_{i j}=\Delta \epsilon_{i}^{(\exp )} \rho_{i j} \Delta \epsilon_{j}^{(\exp )} .
$$

As indicated in eq. (C.8), the $\chi^{2}$ depends on five parameters. However, $\xi$ carries a different meaning than the other parameters, since it provides a measure of the fine-tuning of the model. The absolute minimum of the $\chi^{2}$ is in particular obtained for a very small value of $\xi$, which is highly unnatural. We compute therefore the minimum of the $\chi^{2}$ for a fixed value of $\xi$, and require

$$
\left.\chi^{2}\left(\phi_{L}, \phi_{R}, R, m_{\rho}\right)\right|_{\xi}-\left.\chi_{\min }^{2}\right|_{\xi} \leq 13.28
$$

The value on the right-hand side corresponds to a confidence level of $99 \%$ with four degrees of freedom $\left(\phi_{L}, \phi_{R}, R\right.$ and $\left.m_{\rho}\right)$. For the values quoted in section 4.1 , we have

$$
\left.\chi_{\min }^{2}\right|_{\xi=0.25} \cong 0.98,\left.\quad \chi_{\min }^{2}\right|_{\xi=0.1} \cong 0.85 \text {. }
$$

Note that the minimum of the $\chi^{2}$ in the MCHM5 is significantly lower than in the Standard Model, $\chi_{\mathrm{SM}}^{2}=5.03$, which is expected due to the larger number of fitting parameters.

\section{Partial decay widths of heavy fermions in MCHM5}

In this section we collect the formulae for the partial decay widths of fermionic resonances in MCHM5. We start by defining the relevant couplings. We denote by $U_{L, R}$ the transformations that diagonalize the mass matrix in the top sector,

$$
\mathcal{M} \rightarrow U_{L}^{T} \mathcal{M} U_{R}=\operatorname{diag}\left(m_{1}, m_{2}, m_{3}, m_{4}\right),
$$

where $\mathcal{M}$ is the mass matrix in the basis where the rotations in eq. (4.15) have already been performed. ${ }^{17}$ On the other hand, $\bar{G}_{h f \bar{f}}$ is the Yukawa coupling matrix after the rotations in eq. (4.15), and $G_{L, R}^{Z}$ are the matrices containing the couplings of the fermions to the $Z$ boson,

$$
\begin{aligned}
& G_{L}^{Z}=\operatorname{diag}\left(\frac{1}{2}-\frac{2}{3} s_{w}^{2}, \frac{1}{2}-\frac{2}{3} s_{w}^{2},-\frac{1}{2}-\frac{2}{3} s_{w}^{2},-\frac{2}{3} s_{w}^{2}\right) \\
& G_{R}^{Z}=\operatorname{diag}\left(-\frac{2}{3} s_{w}^{2}, \frac{1}{2}-\frac{2}{3} s_{w}^{2},-\frac{1}{2}-\frac{2}{3} s_{w}^{2},-\frac{2}{3} s_{w}^{2}\right)
\end{aligned}
$$

for left-handed and right-handed fields, respectively (the ordering of the fields is understood to be that of eq. (4.14)). Notice that the rotations in eq. (4.15) leave $G_{L, R}^{Z}$ invariant, because they only mix states with the same EW quantum numbers.

\footnotetext{
${ }^{17}$ The mass eigenstates $f_{i L, R}(i=1, \ldots, 4)$ are ordered by decreasing mass, so $f_{4}$ is identified with the top, and $f_{3}$ with the lightest top partner.
} 


\section{D.1 Charge 2/3 states}

We have for the lightest top partner $\psi$ the partial decay width into a $Z$ boson and a top quark

$$
\begin{gathered}
\Gamma(\psi \rightarrow Z t)=\frac{M_{\psi}}{32 \pi} \sqrt{\zeta_{Z t}}\left\{\left(\lambda_{Z L}^{2}+\lambda_{Z R}^{2}\right)\left(\frac{M_{\psi}^{2}}{m_{Z}^{2}}\right)\left[\frac{m_{Z}^{2}\left(M_{\psi}^{2}+m_{t}^{2}\right)+\left(M_{\psi}^{2}-m_{t}^{2}\right)^{2}-2 m_{Z}^{4}}{M_{\psi}^{4}}\right]\right. \\
\left.-12 \frac{m_{t}}{M_{\psi}} \lambda_{Z L} \lambda_{Z R}\right\}
\end{gathered}
$$

where

$$
\zeta_{Z t}=1-2 \frac{m_{t}^{2}+m_{Z}^{2}}{M_{\psi}^{2}}+\frac{\left(m_{t}^{2}-m_{Z}^{2}\right)^{2}}{M_{\psi}^{4}}
$$

and

$$
\lambda_{Z L}=g_{Z}\left(U_{L}^{T} G_{L}^{Z} U_{L}\right)_{34}, \quad \lambda_{Z R}=g_{Z}\left(U_{R}^{T} G_{L}^{Z} U_{R}\right)_{34},
$$

where $g_{Z} \equiv g / \cos \theta_{w}$. From the Yukawa Lagrangian eq. (4.26) we can extract the leadingorder couplings of $\tilde{T}, T, X^{2 / 3}$. They are given by

$$
\begin{array}{rlrl}
\lambda_{Z L}^{\tilde{T}}=\frac{y}{\sqrt{2}} s_{L} c_{R} \frac{m_{Z}}{M_{\tilde{T}}}, & \lambda_{Z R}^{\tilde{T}}=0 ; & \lambda_{Z L}^{X^{2 / 3}}=0, \quad \lambda_{Z R}^{X^{2 / 3}}=\frac{y}{\sqrt{2}} s_{R} \frac{m_{Z}}{M_{X^{2 / 3}}} ; \\
\lambda_{Z L}^{T}=0, & \lambda_{Z R}^{T}=\frac{y}{\sqrt{2}} s_{R} c_{L} \frac{m_{Z}}{M_{T}} .
\end{array}
$$

On the other hand for the decay $\psi \rightarrow W b$ we find (neglecting $m_{b}$ )

$$
\Gamma(\psi \rightarrow W b)=\frac{M_{\psi}}{32 \pi} \lambda_{W L}^{2}\left(\frac{M_{\psi}^{2}}{m_{W}^{2}}\right)\left(1-3 \frac{m_{W}^{4}}{M_{\psi}^{4}}+2 \frac{m_{W}^{6}}{M_{\psi}^{6}}\right),
$$

where $\lambda_{W L}=(g / \sqrt{2})\left(U_{L}\right)_{13}$. The leading order couplings read

$$
\lambda_{W L}^{\tilde{T}}=y s_{L} c_{R} \frac{m_{W}}{M_{\tilde{T}}} ; \quad \lambda_{W L}^{X^{2 / 3}}=0 ; \quad \lambda_{W L}^{T}=0 .
$$

For the decay $\psi \rightarrow h t$ we find

$$
\Gamma(\psi \rightarrow h t)=\frac{M_{\psi}}{32 \pi} \sqrt{\zeta_{h t}}\left[\left(\lambda_{h L}^{2}+\lambda_{h R}^{2}\right)\left(1+\frac{m_{t}^{2}}{M_{\psi}^{2}}-\frac{m_{h}^{2}}{M_{\psi}^{2}}\right)+4 \frac{m_{t}}{M_{\psi}} \lambda_{h L} \lambda_{h R}\right]
$$

where

$$
\zeta_{h t}=1-2 \frac{m_{t}^{2}+m_{h}^{2}}{M_{\psi}^{2}}+\frac{\left(m_{t}^{2}-m_{h}^{2}\right)^{2}}{M_{\psi}^{4}}
$$

and

$$
\lambda_{h L}=-y\left(U_{L}^{T} \bar{G}_{h f \bar{f}} U_{R}\right)_{43}, \quad \lambda_{h R}=-y\left(U_{L}^{T} \bar{G}_{h f \bar{f}} U_{R}\right)_{34} .
$$

The leading order couplings read

$$
\begin{array}{rlrl}
\lambda_{h L}^{\tilde{T}}=\frac{y}{\sqrt{2}} s_{L} c_{R}, & \lambda_{h R}^{\tilde{T}}=0 ; & \lambda_{h L}^{X^{2 / 3}}=0, \quad \lambda_{h R}^{X^{2 / 3}}=\frac{y}{\sqrt{2}} s_{R} ; \\
\lambda_{h L}^{T}=0, & \lambda_{h R}^{T}=\frac{y}{\sqrt{2}} s_{R} c_{L} .
\end{array}
$$




\section{D.2 $B$ and $X^{5 / 3}$}

For $\chi=X^{5 / 3}, B$ we find for the decay width into a $W$ boson and a top quark

$$
\begin{gathered}
\Gamma\left(\chi \rightarrow W^{ \pm} t\right)=\frac{M_{\chi}}{32 \pi} \sqrt{\zeta_{W t}}\left\{\left(\lambda_{\chi L}^{2}+\lambda_{\chi R}^{2}\right)\left(\frac{M_{\chi}^{2}}{m_{W}^{2}}\right)\left[\frac{m_{W}^{2}\left(M_{\chi}^{2}+m_{t}^{2}\right)+\left(M_{\chi}^{2}-m_{t}^{2}\right)^{2}-2 m_{W}^{4}}{M_{\chi}^{4}}\right]\right. \\
\left.-12 \frac{m_{t}}{M_{\chi}} \lambda_{\chi L} \lambda_{\chi R}\right\}
\end{gathered}
$$

where

$$
\zeta_{W t}=1-2 \frac{m_{t}^{2}+m_{W}^{2}}{M_{\chi}^{2}}+\frac{\left(m_{t}^{2}-m_{W}^{2}\right)^{2}}{M_{\chi}^{4}}
$$

and

$$
\begin{aligned}
\lambda_{X^{5 / 3} L} & =\frac{g}{\sqrt{2}}\left(U_{L}\right)_{34}, & \lambda_{X^{5 / 3} R} & =\frac{g}{\sqrt{2}}\left(U_{R}\right)_{34} \\
\lambda_{B L} & =\frac{g}{\sqrt{2}}\left(U_{L}\right)_{24}, & \lambda_{B R} & =\frac{g}{\sqrt{2}}\left(U_{R}\right)_{24} .
\end{aligned}
$$

The leading order couplings are

$$
\lambda_{X^{5 / 3} L}=0, \quad \lambda_{X^{5 / 3} R}=y s_{R} \frac{m_{W}}{M_{5 / 3}} ; \quad \lambda_{B L}=0, \quad \lambda_{B R}=y s_{R} c_{L} \frac{m_{W}}{M_{B}} .
$$

The leading order expressions of the heavy fermion masses are reported in eq. (4.16).

The formulae for the partial decay widths contained in this appendix reduce to those given in ref. [102] when the approximations in eqs. (D.7), (D.8), (D.10), (D.14), (D.15), (D.20) are made.

\section{E Analytical results for the $g g \rightarrow h h$ cross section in MCHM5}

We present here the analytical result for the partonic gluon fusion cross section into two Higgs bosons, $\hat{\sigma}_{g g \rightarrow h h}$, in MCHM5.

\section{E.1 Notation}

The four-momenta of the gluons are denoted by $p_{1}$ and $p_{2}$, and the four-momenta of the Higgs bosons by $p_{3}$ and $p_{4}$. All momenta are taken incoming. The Mandelstam variables $\hat{s}, \hat{t}, \hat{u}$ are given by

$$
\hat{s}=\left(p_{1}+p_{2}\right)^{2} \quad \hat{t}=\left(p_{1}+p_{3}\right)^{2} \quad \hat{u}=\left(p_{2}+p_{3}\right)^{2} .
$$

The scalar integrals are defined as

$$
\begin{aligned}
& C_{i j}\left(m_{1}^{2}, m_{2}^{2}, m_{3}^{2}\right)= \\
& \int \frac{\mathrm{d}^{4} q}{i \pi^{2}} \overline{\left(q^{2}-m_{1}^{2}\right)\left(\left(q+p_{i}\right)^{2}-m_{2}^{2}\right)\left(\left(q+p_{i}+p_{j}\right)^{2}-m_{3}^{2}\right)} \\
D_{i j k}\left(m_{1}^{2}, m_{2}^{2}, m_{3}^{2}, m_{4}^{2}\right)= & 1 \\
& \int \frac{\mathrm{d}^{4} q}{i \pi^{2}} \frac{1}{\left(q^{2}-m_{1}^{2}\right)\left(\left(q+p_{i}\right)^{2}-m_{2}^{2}\right)\left(\left(q+p_{i}+p_{j}\right)^{2}-m_{3}^{2}\right)\left(\left(q+p_{i}+p_{j}+p_{k}\right)^{2}-m_{4}^{2}\right)} .
\end{aligned}
$$

The analytic expressions can be found in refs. [103, 104]. They have been evaluated numerically in our code with the help of LoopTools [105]. 


\section{E.2 Tensor basis and projectors}

The tensor basis, which has been given in ref. [32], reads

$$
\begin{aligned}
A_{1}^{\mu \nu} & =g^{\mu \nu}-\frac{p_{1}^{\nu} p_{2}^{\mu}}{\left(p_{1} \cdot p_{2}\right)} \\
A_{2}^{\mu \nu} & =g^{\mu \nu}+\frac{p_{3}^{2} p_{1}^{\nu} p_{2}^{\mu}}{p_{T}^{2}\left(p_{1} \cdot p_{2}\right)}-\frac{2\left(p_{3} \cdot p_{2}\right) p_{1}^{\nu} p_{3}^{\mu}}{p_{T}^{2}\left(p_{1} \cdot p_{2}\right)}-\frac{2\left(p_{3} \cdot p_{1}\right) p_{3}^{\nu} p_{2}^{\mu}}{p_{T}^{2}\left(p_{1} \cdot p_{2}\right)}+\frac{2 p_{3}^{\mu} p_{3}^{\nu}}{p_{T}^{2}} \\
\text { with } \quad p_{T}^{2} & =2 \frac{\left(p_{1} \cdot p_{3}\right)\left(p_{2} \cdot p_{3}\right)}{\left(p_{1} \cdot p_{2}\right)}-p_{3}^{2} \\
\text { and } \quad A_{1} \cdot A_{2} & =0 \quad \text { and } A_{1} \cdot A_{1}=A_{2} \cdot A_{2}=2 .
\end{aligned}
$$

\section{E.3 Triangle form factor}

The triangle form factor can be cast into the form

$$
F_{\triangle}(m)=2\left[2 m+\left(4 m^{3}-\hat{s} m\right) C_{12}\left(m^{2}, m^{2}, m^{2}\right)\right]
$$

and can be found in ref. [33]. In the limit of large quark mass $m \gg \sqrt{\hat{s}} \sim m_{h}$ the triangle form factor simplifies to $F_{\triangle}=2 \hat{s} /(3 m)$. This is equivalent to applying the low-energy theorem. The corresponding amplitude is given by

$$
\mathcal{A}_{\triangle}=\frac{\alpha_{s}}{4 \pi} A_{1}^{\mu \nu} \epsilon_{\mu}^{a} \epsilon_{\nu}^{b} \delta_{a b} \sum_{i=1}^{4} \underbrace{\left(\frac{1}{\hat{s}-m_{h}^{2}} g_{h h h} g_{h \bar{q}_{i} q_{i}}+2 g_{h h \bar{q}_{i} q_{i}}\right)}_{C_{i, \Delta}} F_{\triangle}\left(m_{i}\right) .
$$

The couplings $g_{h \bar{q}_{i} q_{i}}$ and $g_{h h \bar{q}_{i} q_{i}}$ are the diagonal elements obtained from the Higgs coupling matrices $y G_{h f \bar{f}}$ in eq. (4.19) and $y /(2 f) G_{h h f \bar{f}}$ in eq. (4.20), respectively, after rotation to the mass eigenstate basis. The triple Higgs coupling $g_{h h h}$ is given in the MCHM5 by

$$
g_{h h h}=\frac{3 m_{h}^{2}}{v} \frac{1-2 \xi}{\sqrt{1-\xi}} .
$$

In the SM limit, in eq. (E.6) there is no sum over heavy top partners, and we are only left with the top quark contribution with the Higgs coupling to the tops given by $g_{h \bar{t} t}=m_{t} / v$, and $g_{h h \bar{t} t}=0$. The triple Higgs coupling in the SM limit can be obtained from eq. (E.7) by setting $\xi=0$.

\section{E.4 Box form factors}

In the box diagrams we can have spin $S_{z}=0$ and 2 gluon gluon couplings. The matrix elements can therefore be written in terms of two gauge invariant form factors. Furthermore, we have divided the form factors for the boxes into the parts which do not involve a $\gamma_{5}$ and the parts which are proportional to two $\gamma_{5}$ matrices. Couplings with a $\gamma_{5}$ arise only for Higgs couplings to two different fermions (but of same flavour). The diagrams including only one $\gamma_{5}$ vanish because of the sign flip of the coupling when the direction of the fermion line changes. The form factors have been calculated with FeynCalc [106] and checked against ref. [107]. For the limit $m_{1} \rightarrow m_{2}$ they agree with ref. [33]. The form 
factors are UV-finite as the coefficients in front of the UV-divergent one- and two-point functions $A_{0}$ and $B_{0}$ are anti-symmetric in $m_{i}$ and $m_{j}$ and vanish upon summation over $i, j$.

We introduce the following abbreviations

$$
\begin{array}{rlrl}
C_{12} & \equiv C_{12}\left(m_{1}^{2}, m_{1}^{2}, m_{1}^{2}\right) & & C_{13} \equiv C_{13}\left(m_{1}^{2}, m_{1}^{2} \cdot m_{2}^{2}\right) \\
C_{14} & \equiv C_{14}\left(m_{1}^{2}, m_{1}^{2}, m_{2}^{2}\right) & C_{23} \equiv C_{23}\left(m_{1}^{2}, m_{1}^{2}, m_{2}^{2}\right) \\
C_{24} & \equiv C_{24}\left(m_{1}^{2}, m_{1}^{2}, m_{2}^{2}\right) & C_{34} \equiv C_{34}\left(m_{1}^{2}, m_{1}^{2}, m_{2}^{2}\right) \\
D_{123} & \equiv D_{123}\left(m_{1}^{2}, m_{1}^{2}, m_{1}^{2}, m_{2}^{2}\right) & D_{132} & \equiv D_{132}\left(m_{1}^{2}, m_{1}^{2}, m_{2}^{2}, m_{2}^{2}\right) \\
D_{213} & \equiv D_{213}\left(m_{1}^{2}, m_{1}^{2}, m_{1}^{2}, m_{2}^{2}\right) . & &
\end{array}
$$

The box form factors $F_{\square}$ and $G_{\square}$ associated with spin 0 and spin 2, respectively, are then given by (contributions which cancel by summing up all contributions are omitted)

$$
\begin{aligned}
& F_{\square}\left(m_{i}, m_{j}\right) \\
& =\frac{2}{\hat{s}}\left[2 \hat{s}+4 m_{i}^{2} \hat{s} C_{12}+\hat{s}\left(\left(m_{i}+m_{j}\right)\left(2 m_{i}^{2}\left(m_{i}+m_{j}\right)-m_{i} \hat{s}\right)-m_{i}^{2}(\hat{t}+\hat{u})\right)\left(D_{123}+D_{132}+D_{213}\right)\right. \\
& +\left(m_{h}^{2}-\left(m_{i}+m_{j}\right)^{2}\right)\left[\left(\hat{t}-m_{h}^{2}\right)\left(C_{13}+C_{24}\right)+\left(\hat{u}-m_{h}^{2}\right)\left(C_{23}+C_{14}\right)\right. \\
& \left.\left.-\left(\hat{t} \hat{u}-m_{h}^{4}+\hat{s}\left(m_{j}^{2}-m_{i}^{2}\right)\right) D_{132}\right]\right] \\
& G_{\square}\left(m_{i}, m_{j}\right) \\
& =\frac{1}{\hat{t} \hat{u}-m_{h}^{4}}\left[\left(\hat{t}^{2}+\hat{u}^{2}-\left(4 m_{j}^{2}+4 m_{i} m_{j}\right)(\hat{t}+\hat{u})+4\left(m_{j}-m_{i}\right)\left(m_{i}+m_{j}\right)^{3}+2 m_{h}^{4}\right) \hat{s} C_{12}\right. \\
& +\left(m_{h}^{4}+\hat{t}^{2}-2 \hat{t}\left(m_{i}+m_{j}\right)^{2}\right)\left(\left(\hat{t}-m_{h}^{2}\right)\left(C_{13}+C_{24}\right)-\hat{s} \hat{t} D_{213}\right) \\
& +\left(m_{h}^{4}+\hat{u}^{2}-2 \hat{u}\left(m_{i}+m_{j}\right)^{2}\right)\left(\left(\hat{u}-m_{h}^{2}\right)\left(C_{23}+C_{14}\right)-\hat{s} \hat{u} D_{123}\right) \\
& -\left(\hat{t}^{2}+\hat{u}^{2}-2 m_{h}^{4}\right)\left(\hat{t}+\hat{u}-2\left(m_{i}+m_{j}\right)^{2}\right) C_{34} \\
& \left.-\left(\hat{t}+\hat{u}-2\left(m_{i}+m_{j}\right)^{2}\right)\left(\left(\hat{t} \hat{u}-m_{h}^{4}\right)\left(m_{i}^{2}+m_{j}^{2}\right)+\hat{s}\left(m_{i}^{2}-m_{j}^{2}\right)^{2}\right)\left(D_{123}+D_{132}+D_{213}\right)\right]
\end{aligned}
$$

and

$$
F_{\square, 5}\left(m_{i}, m_{j}\right)=-F_{\square}\left(m_{i},-m_{j}\right), \quad G_{\square, 5}\left(m_{i}, m_{j}\right)=-G_{\square}\left(m_{i},-m_{j}\right) .
$$

Here $F_{\square, 5}$ and $G_{\square, 5}$ denote the spin 0 and 2 box form factors which are proportional to the Higgs couplings to quarks, $g_{h \bar{q}_{i} q_{j}, 5}$, containing a $\gamma_{5}$ matrix. In the large quark mass limit for $m_{i}=m_{j}$ the form factors reduce to $F_{\square}=-2 \hat{s} /\left(3 m_{i}^{2}\right)$ and $G_{\square}=0 .{ }^{18}$ The spin 0 and spin 2 box amplitudes read

$$
\mathcal{A}_{0, \square}=\frac{\alpha_{s}}{4 \pi} \epsilon_{\mu}^{a} \epsilon_{\nu}^{b} \delta_{a b} A_{1}^{\mu \nu}\left(\sum_{i=1}^{4} \sum_{j=1}^{4} g_{h \bar{q}_{i} q_{j}}^{2} F_{\square}\left(m_{i}, m_{j}\right)+\sum_{i=1}^{4} \sum_{j=1}^{4} g_{h \bar{q}_{i} q_{j}, 5}^{2} F_{\square, 5}\left(m_{i}, m_{j}\right)\right)
$$

and

$$
\mathcal{A}_{2, \square}=\frac{\alpha_{s}}{4 \pi} \epsilon_{\mu}^{a} \epsilon_{\nu}^{b} \delta_{a b} A_{2}^{\mu \nu}\left(\sum_{i=1}^{4} \sum_{j=1}^{4} g_{h \bar{q}_{i} q_{j}}^{2} G_{\square}\left(m_{i}, m_{j}\right)+\sum_{i=1}^{4} \sum_{j=1}^{4} g_{h \bar{q}_{i} q_{j}, 5}^{2} G_{\square, 5}\left(m_{i}, m_{j}\right)\right) .
$$

\footnotetext{
${ }^{18}$ For $m_{i}=m_{j}$ the couplings in front of the form factors $F_{\square, 5}$ and $G_{\square, 5}$ vanish, so that in this case these form factors are not needed.
} 
The couplings $g_{h \bar{q}_{i} q_{j}}$ and $g_{h \bar{q}_{i} q_{j}, 5}$ are given by

$$
\begin{aligned}
g_{h \bar{q}_{i} q_{j}} & =\frac{y}{2}\left(\tilde{G}_{h f f, i j}+\tilde{G}_{h f f, j i}\right) \\
g_{h \bar{q}_{i} q_{j}, 5} & =\frac{y}{2}\left(\tilde{G}_{h f f, i j}-\tilde{G}_{h f f, j i}\right),
\end{aligned}
$$

where $\tilde{G}_{h f f, i j}$ denotes the (ith, $j$ th) matrix element of the coupling matrix of eq. (4.19) in the mass eigenstate basis. Note that $g_{h \bar{q}_{i} q_{j}, 5}$ is antisymmetric in $i$ and $j$ and hence changes sign, if incoming and outgoing fermions in the vertex are interchanged.

The complete amplitude of the process is given by

$$
\mathcal{A}(g g \rightarrow h h)=\mathcal{A}_{\triangle}+\mathcal{A}_{0, \square}+\mathcal{A}_{2, \square}
$$

\section{E.5 Expansion of the form factors}

In the case where top partners are neglected, we can perform explicitly the expansion of the form factors in $1 / m_{t}^{2}$, i.e. for small external momenta (see ref. [108]), and go beyond the leading order, the latter corresponding to the LET result. We find

$$
\begin{aligned}
F_{\triangle} & =\frac{\hat{s}}{m_{t}}\left(\frac{2}{3}+\frac{7}{180} \frac{\hat{s}}{m_{t}^{2}}\right), \\
F_{\square} & =\frac{\hat{s}}{m_{t}^{2}}\left(-\frac{2}{3}-\frac{7}{30} \frac{m_{h}^{2}}{m_{t}^{2}}\right), \\
G_{\square} & =\frac{\hat{s}}{m_{t}^{4}} \frac{11}{90}\left(\frac{m_{h}^{4}-\hat{t} \hat{u}}{\hat{s}}\right) .
\end{aligned}
$$

The partonic cross section then reads

$$
\hat{\sigma}_{g g \rightarrow h h}=\frac{\alpha_{s}^{2}}{1024(2 \pi)^{3}} \frac{1}{\hat{s}^{2}} \int_{\hat{t}_{-}}^{\hat{t}_{+}} d \hat{t}\left[\left|C_{\triangle} F_{\triangle}+C_{\square} F_{\square}\right|^{2}+\left|C_{\square} G_{\square}\right|^{2}\right]
$$

with $\hat{t}_{ \pm}$given by eq. (6.3) and

$$
\begin{aligned}
\left|C_{\triangle} F_{\triangle}+C_{\square} F_{\square}\right|^{2}+\left|C_{\square} G_{\square}\right|^{2} & =\frac{\hat{s}^{2}}{v^{4}} \frac{4}{9}\left(c_{\triangle}-c_{\square}\right)^{2}\left[1+\frac{1}{m_{t}^{2}\left(c_{\triangle}-c_{\square}\right)}\left(c_{\triangle} \frac{7}{60} \hat{s}-c_{\square} \frac{7}{10} m_{h}^{2}\right)\right] \\
c_{\triangle} & =\frac{3 m_{h}^{2}}{\hat{s}-m_{h}^{2}}\left(\frac{1-2 \xi}{\sqrt{1-\xi}}\right)^{2}-4 \xi, \quad c_{\square}=\left(\frac{1-2 \xi}{\sqrt{1-\xi}}\right)^{2},
\end{aligned}
$$

where we used

$$
C_{\triangle}=\left(\frac{1}{\hat{s}-m_{h}^{2}} g_{h h h} g_{h \bar{t} t}+2 g_{h h \bar{t} t}\right) \equiv \frac{m_{t}}{v^{2}} c_{\triangle}, \quad C_{\square}=g_{h \bar{t} t}^{2} \equiv \frac{m_{t}^{2}}{v^{2}} c_{\square},
$$

with the couplings given by $g_{h \bar{t} t}=\left(m_{t} / v\right)(1-2 \xi) / \sqrt{1-\xi}, g_{h h \bar{t} t}=-2 m_{t} \xi / v^{2}$ and $g_{h h h}$ as defined in eq. (E.7). The leading term in $1 / m_{t}^{2}$ corresponds to the LET result, see eqs. (3.22) and (6.1).

Open Access. This article is distributed under the terms of the Creative Commons Attribution License which permits any use, distribution and reproduction in any medium, provided the original author(s) and source are credited. 


\section{References}

[1] CMS collaboration, J. Incandela, Status of the CMS SM Higgs Search, talk at the Latest update in the search for the Higgs boson, CERN, Geneva Switzerland, 4 vJuly 2012, http://indico.cern.ch/conferenceDisplay.py?confId=197461.

[2] ATLAS collaboration, F. Gianotti, Status of Standard Model Higgs Searches in ATLAS, talk at the Latest update in the search for the Higgs boson, CERN, Geneva Switzerland, 4 vJuly 2012, http://indico.cern.ch/conferenceDisplay.py?confId=197461.

[3] D. Carmi, A. Falkowski, E. Kuflik and T. Volansky, Interpreting LHC Higgs results from natural new physics perspective, JHEP 07 (2012) 136 [arXiv:1202.3144] [INSPIRE].

[4] A. Azatov, R. Contino and J. Galloway, Model-independent bounds on a light Higgs, JHEP 04 (2012) 127 [arXiv: 1202.3415] [INSPIRE].

[5] J. Espinosa, C. Grojean, M. Muhlleitner and M. Trott, Fingerprinting Higgs suspects at the LHC, JHEP 05 (2012) 097 [arXiv: 1202.3697] [INSPIRE].

[6] P.P. Giardino, K. Kannike, M. Raidal and A. Strumia, Reconstructing Higgs boson properties from the LHC and Tevatron data, JHEP 06 (2012) 117 [arXiv: 1203.4254] [INSPIRE].

[7] J. Ellis and T. You, Global analysis of experimental constraints on a possible Higgs-like particle with mass 125 GeV, JHEP 06 (2012) 140 [arXiv: 1204.0464] [INSPIRE].

[8] M. Klute, R. Lafaye, T. Plehn, M. Rauch and D. Zerwas, Measuring Higgs Couplings from LHC Data, arXiv:1205.2699 [INSPIRE].

[9] R. Barbieri and G. Giudice, Upper bounds on supersymmetric particle masses, Nucl. Phys. B 306 (1988) 63 [INSPIRE].

[10] O. Matsedonskyi, G. Panico and A. Wulzer, Light top partners for a light composite Higgs, arXiv: 1204.6333 [INSPIRE].

[11] M. Redi and A. Tesi, Implications of a Light Higgs in Composite Models, arXiv:1205.0232 [INSPIRE].

[12] D. Marzocca, M. Serone and J. Shu, General composite Higgs models, JHEP 08 (2012) 013 [arXiv:1205.0770] [INSPIRE].

[13] A. Pomarol and F. Riva, The composite Higgs and light resonance connection, JHEP 08 (2012) 135 [arXiv:1205.6434] [INSPIRE].

[14] C. Dennis, M. Karagoz, G. Servant and J. Tseng, Multi-W events at LHC from a warped extra dimension with custodial symmetry, hep-ph/0701158 [INSPIRE].

[15] R. Contino and G. Servant, Discovering the top partners at the LHC using same-sign dilepton final states, JHEP 06 (2008) 026 [arXiv:0801.1679] [INSPIRE].

[16] J. Aguilar-Saavedra, Identifying top partners at LHC, JHEP 11 (2009) 030 [arXiv:0907.3155] [INSPIRE].

[17] J. Mrazek and A. Wulzer, A strong sector at the LHC: top partners in same-sign dileptons, Phys. Rev. D 81 (2010) 075006 [arXiv:0909.3977] [INSPIRE].

[18] G. Dissertori, E. Furlan, F. Moortgat and P. Nef, Discovery potential of top-partners in a realistic composite Higgs model with early LHC data, JHEP 09 (2010) 019 [arXiv: 1005.4414] [INSPIRE]. 
[19] K. Harigaya, S. Matsumoto, M.M. Nojiri and K. Tobioka, Search for the Top partner at the LHC using Multi-b-Jet channels, Phys. Rev. D 86 (2012) 015005 [arXiv:1204.2317] [INSPIRE].

[20] A. Azatov et al., Higgs boson production via vector-like top-partner decays: diphoton or multilepton plus multijets channels at the LHC, Phys. Rev. D 85 (2012) 115022 [arXiv: 1204.0455] [INSPIRE].

[21] N. Vignaroli, Discovering the composite Higgs through the decay of a heavy fermion, JHEP 07 (2012) 158 [arXiv: 1204.0468] [INSPIRE].

[22] J. Berger, J. Hubisz and M. Perelstein, A fermionic top partner: naturalness and the LHC, JHEP 07 (2012) 016 [arXiv: 1205.0013] [INSPIRE].

[23] J.R. Ellis, M.K. Gaillard and D.V. Nanopoulos, A phenomenological profile of the Higgs boson, Nucl. Phys. B 106 (1976) 292 [INSPIRE].

[24] M.A. Shifman, A. Vainshtein, M. Voloshin and V.I. Zakharov, Low-Energy Theorems for Higgs Boson Couplings to Photons, Sov. J. Nucl. Phys. 30 (1979) 711 [Yad. Fiz. 30 (1979) 1368] [INSPIRE].

[25] B.A. Kniehl and M. Spira, Low-energy theorems in Higgs physics, Z. Phys. C 69 (1995) 77 [hep-ph/9505225] [INSPIRE].

[26] G. Giudice, C. Grojean, A. Pomarol and R. Rattazzi, The strongly-interacting light Higgs, JHEP 06 (2007) 045 [hep-ph/0703164] [INSPIRE].

[27] A. Falkowski, Pseudo-goldstone Higgs production via gluon fusion, Phys. Rev. D 77 (2008) 055018 [arXiv: 0711.0828] [INSPIRE].

[28] I. Low and A. Vichi, On the production of a composite Higgs boson, Phys. Rev. D 84 (2011) 045019 [arXiv: 1010.2753] [INSPIRE].

[29] A. Azatov and J. Galloway, Light custodians and Higgs physics in composite models, Phys. Rev. D 85 (2012) 055013 [arXiv:1110.5646] [INSPIRE].

[30] C. Degrande, J. Gerard, C. Grojean, F. Maltoni and G. Servant, Probing top-Higgs non-standard interactions at the LHC, JHEP 07 (2012) 036 [arXiv:1205.1065] [INSPIRE].

[31] A. Carmona, M. Chala and J. Santiago, New Higgs production mechanism in composite Higgs models, JHEP 07 (2012) 049 [arXiv: 1205.2378] [INSPIRE].

[32] E.N. Glover and J. van der Bij, Higgs boson pair production via gluon fusion, Nucl. Phys. B 309 (1988) 282 [INSPIRE].

[33] T. Plehn, M. Spira and P. Zerwas, Pair production of neutral Higgs particles in gluon-gluon collisions, Nucl. Phys. B 479 (1996) 46 [Erratum ibid. B 531 (1998) 655] [hep-ph/9603205] [INSPIRE].

[34] U. Baur, T. Plehn and D.L. Rainwater, Measuring the Higgs boson self coupling at the LHC and finite top mass matrix elements, Phys. Rev. Lett. 89 (2002) 151801 [hep-ph/0206024] [INSPIRE].

[35] C.O. Dib, R. Rosenfeld and A. Zerwekh, Double Higgs production and quadratic divergence cancellation in little Higgs models with T parity, JHEP 05 (2006) 074 [hep-ph/0509179] [INSPIRE].

[36] R. Gröber and M. Mühlleitner, Composite Higgs boson pair production at the LHC, JHEP 06 (2011) 020 [arXiv: 1012.1562] [INSPIRE]. 
[37] R. Contino, M. Ghezzi, M. Moretti, G. Panico, F. Piccinini and A. Wulzer, Anomalous couplings in double Higgs production, JHEP 08 (2012) 154 [arXiv: 1205.5444] [INSPIRE].

[38] R. Contino, C. Grojean, M. Moretti, F. Piccinini and R. Rattazzi, Strong double Higgs production at the LHC, JHEP 05 (2010) 089 [arXiv:1002.1011] [INSPIRE].

[39] M. Farina, C. Grojean and E. Salvioni, (Dys)Zphilia or a custodial breaking Higgs at the LHC, JHEP 07 (2012) 012 [arXiv:1205.0011] [INSPIRE].

[40] K. Agashe, R. Contino and A. Pomarol, The minimal composite Higgs model, Nucl. Phys. B 719 (2005) 165 [hep-ph/0412089] [INSPIRE].

[41] R. Contino, L. Da Rold and A. Pomarol, Light custodians in natural composite Higgs models, Phys. Rev. D 75 (2007) 055014 [hep-ph/0612048] [INSPIRE].

[42] R. Contino, Y. Nomura and A. Pomarol, Higgs as a holographic pseudoGoldstone boson, Nucl. Phys. B 671 (2003) 148 [hep-ph/0306259] [INSPIRE].

[43] K. Agashe and R. Contino, The minimal composite Higgs model and electroweak precision tests, Nucl. Phys. B 742 (2006) 59 [hep-ph/0510164] [INSPIRE].

[44] K. Agashe, R. Contino, L. Da Rold and A. Pomarol, A custodial symmetry for Z $b \bar{b}$, Phys. Lett. B 641 (2006) 62 [hep-ph/0605341] [INSPIRE].

[45] J. Espinosa, C. Grojean and M. Muhlleitner, Composite Higgs search at the LHC, JHEP 05 (2010) 065 [arXiv: 1003.3251] [INSPIRE].

[46] J. Espinosa, C. Grojean and M. Muhlleitner, Composite Higgs under LHC experimental scrutiny, EPJ Web Conf. 28 (2012) 08004 [arXiv:1202.1286] [INSPIRE].

[47] I. Low, R. Rattazzi and A. Vichi, Theoretical constraints on the Higgs effective couplings, JHEP 04 (2010) 126 [arXiv:0907.5413] [INSPIRE].

[48] K. Hagiwara and H. Murayama, Multiple weak boson production via gluon fusion, Phys. Rev. D 41 (1990) 1001 [INSPIRE].

[49] A. Pierce, J. Thaler and L.-T. Wang, Disentangling dimension six operators through Di-Higgs boson production, JHEP 05 (2007) 070 [hep-ph/0609049] [INSPIRE].

[50] H. Georgi, S. Glashow, M. Machacek and D.V. Nanopoulos, Higgs bosons from two gluon annihilation in proton proton collisions, Phys. Rev. Lett. 40 (1978) 692 [INSPIRE].

[51] A. Djouadi, The anatomy of electro-weak symmetry breaking. I: the Higgs boson in the standard model, Phys. Rept. 457 (2008) 1 [hep-ph/0503172] [INSPIRE].

[52] LhC Higgs Cross Section Working Group collaboration, S. Dittmaier et al., Handbook of LHC Higgs Cross Sections: 1. Inclusive Observables, arXiv:1101.0593 [INSPIRE].

[53] E. Furlan, Gluon-fusion Higgs production at NNLO for a non-standard Higgs sector, JHEP 10 (2011) 115 [arXiv:1106.4024] [INSPIRE].

[54] A. Djouadi, W. Kilian, M. Mühlleitner and P. Zerwas, Production of neutral Higgs boson pairs at LHC, Eur. Phys. J. C 10 (1999) 45 [hep-ph/9904287] [InSPIRE].

[55] A. Djouadi, W. Kilian, M. Mühlleitner and P. Zerwas, The reconstruction of trilinear Higgs couplings, hep-ph/0001169 [INSPIRE].

[56] M.M. Mühlleitner, Higgs particles in the standard model and supersymmetric theories, hep-ph/0008127 [INSPIRE]. 
[57] M. Mühlleitner, Testing Higgs selfcouplings at high-energy linear colliders, hep-ph/0101262 [INSPIRE].

[58] A. Martin, W. Stirling, R. Thorne and G. Watt, Parton distributions for the LHC, Eur. Phys. J. C 63 (2009) 189 [arXiv:0901.0002] [InSPIRE].

[59] M.S. Carena, E. Ponton, J. Santiago and C.E. Wagner, Light Kaluza Klein States in Randall-Sundrum Models with Custodial SU(2), Nucl. Phys. B 759 (2006) 202 [hep-ph/0607106] [INSPIRE].

[60] A.D. Medina, N.R. Shah and C.E. Wagner, Gauge-Higgs Unification and Radiative Electroweak Symmetry Breaking in Warped Extra Dimensions, Phys. Rev. D 76 (2007) 095010 [arXiv: 0706 .1281] [INSPIRE].

[61] G. Panico, E. Ponton, J. Santiago and M. Serone, Dark Matter and Electroweak Symmetry Breaking in Models with Warped Extra Dimensions, Phys. Rev. D 77 (2008) 115012 [arXiv:0801.1645] [INSPIRE].

[62] G. Altarelli and R. Barbieri, Vacuum polarization effects of new physics on electroweak processes, Phys. Lett. B 253 (1991) 161 [INSPIRE].

[63] G. Altarelli, R. Barbieri and S. Jadach, Toward a model independent analysis of electroweak data, Nucl. Phys. B 369 (1992) 3 [Erratum ibid. B 376 (1992) 444] [INSPIRE].

[64] G. Altarelli, R. Barbieri and F. Caravaglios, Nonstandard analysis of electroweak precision data, Nucl. Phys. B 405 (1993) 3 [inSPIRE].

[65] AlePh, DELPHi, L3, OPAL, SLD, LeP Electroweak Working Group, SLD Electroweak Group, SLD Heavy Flavour Group collaboration, Precision electroweak measurements on the $Z$ resonance, Phys. Rept. 427 (2006) 257 [hep-ex/0509008] [inSPIRE].

[66] R. Barbieri, B. Bellazzini, V.S. Rychkov and A. Varagnolo, The Higgs boson from an extended symmetry, Phys. Rev. D 76 (2007) 115008 [arXiv:0706.0432] [inSPIRE].

[67] R. Contino, The Higgs as a Composite Nambu-Goldstone Boson, arXiv:1005.4269 [INSPIRE].

[68] P. Lodone, Vector-like quarks in a 'composite' Higgs model, JHEP 12 (2008) 029 [arXiv:0806.1472] [INSPIRE].

[69] M. Gillioz, A light composite Higgs boson facing electroweak precision tests, Phys. Rev. D 80 (2009) 055003 [arXiv:0806 . 3450] [INSPIRE].

[70] C. Anastasiou, E. Furlan and J. Santiago, Realistic Composite Higgs Models, Phys. Rev. D 79 (2009) 075003 [arXiv:0901.2117] [InSPIRE].

[71] CDF, D0 collaboration, T.E.W. Group, Combination of CDF and D0 Measurements of the Single Top Production Cross Section, arXiv:0908.2171 [INSPIRE].

[72] A. Pomarol and J. Serra, Top Quark Compositeness: Feasibility and Implications, Phys. Rev. D 78 (2008) 074026 [arXiv:0806.3247] [INSPIRE].

[73] B. Lillie, J. Shu and T.M. Tait, Top compositeness at the Tevatron and LHC, JHEP 04 (2008) 087 [arXiv:0712.3057] [INSPIRE].

[74] M. Redi and A. Weiler, Flavor and CP invariant composite Higgs models, JHEP 11 (2011) 108 [arXiv: 1106.6357] [INSPIRE]. 
[75] CMS collaboration, S. Chatrchyan et al., Search for quark compositeness in dijet angular distributions from pp collisions at $\sqrt{s}=7$ TeV, JHEP 05 (2012) 055 [arXiv:1202.5535] [INSPIRE].

[76] M. Redi, Composite MFV and Beyond, arXiv:1203.4220 [INSPIRE].

[77] M. Aliev, H. Lacker, U. Langenfeld, S. Moch, P. Uwer and M. Wiedermann, HATHOR: HAdronic Top and Heavy quarks crOss section calculatoR, Comput. Phys. Commun. 182 (2011) 1034 [arXiv:1007.1327] [INSPIRE].

[78] CMS collaboration, Search for $t^{\prime}$ pair production in lepton + jets channel, PAS-EXO-11-099 (2011).

[79] CMS collaboration, S. Chatrchyan et al., Search for heavy, top-like quark pair production in the dilepton final state in pp collisions at $\sqrt{s}=7$ TeV, Phys. Lett. B 716 (2012) 103 [arXiv:1203.5410] [INSPIRE].

[80] CMS collaboration, Search for a Heavy Bottom-like Quark in pp Collisions at $\sqrt{s}=7$ TeV, PAS-EXO-11-036 (2011).

[81] CMS collaboration, S. Chatrchyan et al., Search for heavy bottom-like quarks in 4.9 inverse femtobarns of pp collisions at $\sqrt{s}=7$ TeV, JHEP 05 (2012) 123 [arXiv:1204.1088] [INSPIRE].

[82] CMS collaboration, S. Chatrchyan et al., Search for a Vector-like Quark with Charge $2 / 3$ in $t+Z$ Events from pp Collisions at $\sqrt{s}=7$ TeV, Phys. Rev. Lett. 107 (2011) 271802 [arXiv:1109.4985] [INSPIRE].

[83] ATLAS collaboration, G. Aad et al., Search for pair production of a heavy up-type quark decaying to a $W$ boson and a b-quark in the lepton+jets channel with the ATLAS detector, Phys. Rev. Lett. 108 (2012) 261802 [arXiv:1202.3076] [INSPIRE].

[84] ATLAS collaboration, G. Aad et al., Search for pair-produced heavy quarks decaying to $W q$ in the two-lepton channel at $\sqrt{s}=7$ TeV with the ATLAS detector, Phys. Rev. D 86 (2012) 012007 [arXiv: 1202.3389].

[85] ATLAS collaboration, G. Aad et al., Search for down-type fourth generation quarks with the ATLAS detector in events with one lepton and hadronically decaying $W$ bosons, Phys. Rev. Lett. 109 (2012) 032001 [arXiv:1202.6540] [INSPIRE].

[86] ATLAS collaboration, G. Aad et al., Search for same-sign top-quark production and fourth-generation down-type quarks in pp collisions at $\sqrt{s}=7$ TeV with the ATLAS detector, JHEP 04 (2012) 069 [arXiv:1202.5520] [INSPIRE].

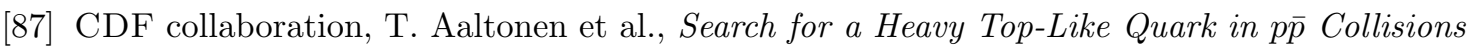
at $\sqrt{s}=1.96$ TeV, Phys. Rev. Lett. 107 (2011) 261801 [arXiv:1107.3875] [INSPIRE].

[88] CDF collaboration, T. Aaltonen et al., Search for heavy bottom-like quarks decaying to an electron or muon and jets in p $\bar{p}$ collisions at $\sqrt{s}=1.96$ TeV, Phys. Rev. Lett. 106 (2011) 141803 [arXiv: 1101.5728] [INSPIRE].

[89] J. Alwall, M. Herquet, F. Maltoni, O. Mattelaer and T. Stelzer, MadGraph 5: going beyond, JHEP 06 (2011) 128 [arXiv:1106.0522] [INSPIRE].

[90] M. Spira, HIGLU: A program for the calculation of the total Higgs production cross-section at hadron colliders via gluon fusion including QCD corrections, hep-ph/9510347 [INSPIRE]. 
[91] S. Dawson, S. Dittmaier and M. Spira, Neutral Higgs boson pair production at hadron colliders: QCD corrections, Phys. Rev. D 58 (1998) 115012 [hep-ph/9805244] [INSPIRE].

[92] U. Baur, T. Plehn and D.L. Rainwater, Determining the Higgs boson selfcoupling at hadron colliders, Phys. Rev. D 67 (2003) 033003 [hep-ph/0211224] [InSPIRE].

[93] U. Baur, T. Plehn and D.L. Rainwater, Examining the Higgs boson potential at lepton and hadron colliders: a comparative analysis, Phys. Rev. D 68 (2003) 033001 [hep-ph/0304015] [INSPIRE].

[94] U. Baur, T. Plehn and D.L. Rainwater, Probing the Higgs selfcoupling at hadron colliders using rare decays, Phys. Rev. D 69 (2004) 053004 [hep-ph/0310056] [INSPIRE].

[95] M.J. Dolan, C. Englert and M. Spannowsky, Higgs self-coupling measurements at the LHC, arXiv:1206.5001 [INSPIRE].

[96] A. Papaefstathiou, L.L. Yang and J. Zurita, Higgs boson pair production at the LHC in the $b \bar{b} W^{+} W^{-}$channel, arXiv:1209.1489 [INSPIRE].

[97] N. Arkani-Hamed, A. Cohen, E. Katz and A. Nelson, The Littlest Higgs, JHEP 07 (2002) 034 [hep-ph/0206021] [INSPIRE].

[98] C. Csáki, J. Hubisz, G.D. Kribs, P. Meade and J. Terning, Variations of little Higgs models and their electroweak constraints, Phys. Rev. D 68 (2003) 035009 [hep-ph/0303236] [INSPIRE].

[99] CDF collaboration, T. Aaltonen et al., Precise measurement of the $W$-boson mass with the CDF II detector, Phys. Rev. Lett. 108 (2012) 151803 [arXiv:1203.0275] [INSPIRE].

[100] D0 collaboration, V.M. Abazov et al., Measurement of the $W$ Boson Mass with the D0 Detector, Phys. Rev. Lett. 108 (2012) 151804 [arXiv:1203.0293] [InSPIRE].

[101] CDF, D0 and Tevatron Electroweak Working Group collaboration, 2012 Update of the Combination of CDF and D0 Results for the Mass of the W Boson, arXiv:1204.0042 [INSPIRE].

[102] C. Bini, R. Contino and N. Vignaroli, Heavy-light decay topologies as a new strategy to discover a heavy gluon, JHEP 01 (2012) 157 [arXiv:1110.6058] [INSPIRE].

[103] G. 't Hooft and M. Veltman, Scalar one loop integrals, Nucl. Phys. B 153 (1979) 365 [INSPIRE].

[104] A. Denner, U. Nierste and R. Scharf, A compact expression for the scalar one loop four point function, Nucl. Phys. B 367 (1991) 637 [InSPIRE].

[105] T. Hahn and M. Pérez-Victoria, Automatized one loop calculations in four-dimensions and D-dimensions, Comput. Phys. Commun. 118 (1999) 153 [hep-ph/9807565] [InSPIRE].

[106] R. Mertig, M. Böhm and A. Denner, FEYNCALC: Computer algebraic calculation of Feynman amplitudes, Comput. Phys. Commun. 64 (1991) 345 [INSPIRE].

[107] T. Figy and R. Zwicky, The other Higgses, at resonance, in the Lee-Wick extension of the Standard Model, JHEP 10 (2011) 145 [arXiv:1108.3765] [INSPIRE].

[108] F. Hoogeveen, The influence of a heavy fermion doublet on Higgs boson production via the gluon fusion mechanism, Nucl. Phys. B 259 (1985) 19 [INSPIRE]. 